\title{
Current economic obstacles to biochar use in agriculture and climate change mitigation
}

Martin Bach, Burkhard Wilske \& Lutz Breuer

\section{Supplement Information}

\section{List of Tables}

Table S1. Yield effect and revenue of biochar soil application in field experiments with agricultural crops.

Table S2. Quantiles of absolute and relative change in crop yield (data of Table S1) from field experiments of biochar application to agricultural crops.

Table S3. Crop market prices used for calculation of revenue.

Table S4. Median and quantiles of the revenue of biochar application to soils (data ref. Table S1).

Table S5. Half-lifes of biochar from soil incubation experiments.

Table S6. Median and quantiles of biochar half-lifes from soil incubation experiments (data ref. Table S5). 
Table S1. Yield effect and revenue of biochar soil application in field experiments with agricultural crops.

\begin{tabular}{|c|c|c|c|c|c|c|c|c|c|c|c|}
\hline $\begin{array}{l}\text { Ref. } \\
\text { No. }\end{array}$ & Crop & Variant $^{\mathrm{a}}$ & $\begin{array}{l}\text { Treatment, plot, site, } \\
\text { year }\end{array}$ & \begin{tabular}{|c|} 
Single/ \\
Multiple \\
(year) $^{b}$
\end{tabular} & $\begin{array}{c}\text { N ferti- } \\
\text { lizing }^{c} \\
\text { (kg N/ha) }\end{array}$ & $\begin{array}{c}\text { Biochar } \\
\text { added } \\
\text { (Mg/ha) }\end{array}$ & $\begin{array}{c}\text { Yield } \\
\text { (Mg/ha) }\end{array}$ & $\begin{array}{l}\text { Rel. } \\
\text { Yield }\end{array}$ & $\begin{array}{l}\text { Signifi- }^{\text {cance }} \\
\text { can }^{-}\end{array}$ & $\begin{array}{c}\text { Yield } \\
\text { change }^{\mathrm{e}} \\
(\mathrm{Mg} / \mathrm{ha})\end{array}$ & $\begin{array}{c}\text { Revenue }^{\mathrm{e}} \\
\text { (USD/ } \\
\text { Mg BC) }\end{array}$ \\
\hline \multirow[t]{12}{*}{1} & Maize & $\mathrm{C}$ & & $\mathrm{S}$ & 0 & & 0.72 & & & & \\
\hline & Maize & $\mathrm{BC} 2$ & & S & 0 & 2.0 & 1.1 & 1.53 & $*$ & 0.38 & 11.24 \\
\hline & Maize & BC4 & & $S$ & 0 & 4.0 & 1.7 & 2.36 & $*$ & 0.98 & 14.50 \\
\hline & Maize & MF30 & & S & 30 & & 0.87 & & & & \\
\hline & Maize & MF30+BC2 & & S & 30 & 2.0 & 1.2 & 1.38 & $*$ & 0.33 & 9.76 \\
\hline & Maize & MF30+BC4 & & S & 30 & 4.0 & 2.26 & 2.60 & $*$ & 1.39 & 20.56 \\
\hline & Maize & MF60 & & S & 60 & & 0.99 & & & & \\
\hline & Maize & MF60+BC2 & & S & 60 & 2.0 & 1.43 & 1.44 & $*$ & 0.44 & 13.02 \\
\hline & Maize & MF60+BC4 & & S & 60 & 4.0 & 2.44 & 2.46 & $*$ & 1.45 & 21.45 \\
\hline & Maize & MF90 & & S & 90 & & 1.05 & & & & \\
\hline & Maize & MF90+BC2 & & S & 90 & 2.0 & 1.68 & 1.60 & $*$ & 0.63 & 18.64 \\
\hline & Maize & MF90+BC4 & & S & 90 & 4.0 & 2.85 & 2.71 & $*$ & 1.80 & 26.63 \\
\hline \multirow[t]{4}{*}{2} & Maize & $\mathrm{MF}$ & & $\bar{S}$ & 150 & & 7.14 & & & & \\
\hline & Maize & $\mathrm{MF}+\mathrm{BC}$ & BC10 & S & 150 & 10.0 & 9.21 & 1.29 & $*$ & 2.07 & 12.25 \\
\hline & Maize & $\mathrm{MF}+\mathrm{Co}$ & & S & 150 & & 7.82 & & & & \\
\hline & Maize & $\mathrm{MF}+\mathrm{BC}+\mathrm{Co}$ & $\mathrm{BC} 2.5$ & S & 150 & 2.5 & 8.57 & 1.10 & n.s. & 0.75 & 17.75 \\
\hline \multirow[t]{6}{*}{3} & Maize & $\mathrm{C}$ & & $\bar{S}$ & $\overline{0}$ & & 2.04 & & & & \\
\hline & Maize & $\mathrm{BC}$ & & S & 0 & 30.0 & 2.39 & 1.17 & $*$ & 0.35 & 0.68 \\
\hline & Maize & FYM & & S & 0 & & 2.40 & & & & \\
\hline & Maize & FYM+BC & & S & 0 & 30.0 & 3.19 & 1.33 & $*$ & 0.79 & 1.56 \\
\hline & Maize & NPK & & S & 150 & & 3.97 & & & & \\
\hline & Maize & $\mathrm{NPK}+\mathrm{BC}$ & & $S$ & 150 & 30.0 & 4.19 & 1.06 & n.s. & 0.22 & 0.43 \\
\hline \multirow[t]{5}{*}{4} & Rice & $\mathrm{C}$ & HK1_Apo & $\bar{S}$ & 0 & & 3.00 & & & & \\
\hline & Rice & $\mathrm{BC}$ & HK1_Apo; BC4 & S & 0 & 4.0 & 2.30 & 0.77 & $*$ & -0.70 & -57.75 \\
\hline & Rice & $\mathrm{BC}$ & HK1_Apo; BC8 & $S$ & 0 & 8.0 & 2.70 & 0.90 & n.s. & -0.30 & -12.38 \\
\hline & Rice & $\mathrm{BC}$ & HK1_Apo; BC16 & S & 0 & 16.0 & 2.20 & 0.73 & $*$ & -0.80 & -16.50 \\
\hline & Rice & MF & HK1_Apo & S & 50 & & 2.80 & & & & \\
\hline
\end{tabular}




\begin{tabular}{|c|c|c|c|c|c|c|c|c|c|c|c|}
\hline $\begin{array}{l}\text { Ref. } \\
\text { No. }\end{array}$ & Crop & Variant $^{\mathrm{a}}$ & $\begin{array}{l}\text { Treatment, plot, site, } \\
\text { year }\end{array}$ & $\begin{array}{c}\text { Single/ } \\
\text { Multiple } \\
\text { (year) }^{\mathrm{b}}\end{array}$ & $\begin{array}{l}\text { N ferti- } \\
\text { lizingc } \\
\text { (kg N/ha) }\end{array}$ & $\begin{array}{l}\text { Biochar } \\
\text { added } \\
\text { (Mg/ha) }\end{array}$ & $\begin{array}{c}\text { Yield } \\
\text { (Mg/ha) }\end{array}$ & $\begin{array}{l}\text { Rel. } \\
\text { Yield }\end{array}$ & $\begin{array}{l}\text { Signifi- } \\
\text { cance }^{d}\end{array}$ & $\begin{array}{c}\text { Yield } \\
\text { change } \\
(\mathrm{Mg} / \mathrm{ha})\end{array}$ & $\begin{array}{c}\text { Revenue }^{\text {e }} \\
\text { (USD/ } \\
\text { Mg BC) }\end{array}$ \\
\hline \multirow{29}{*}{$\begin{array}{c}\mathbf{4} \\
\text { cont'd }^{\star} \mathrm{d}\end{array}$} & Rice & $\mathrm{MF}+\mathrm{BC}$ & HK1_Apo; BC4 & $\mathrm{S}$ & 50 & 4.0 & 3.20 & 1.14 & n.s. & 0.40 & 33.00 \\
\hline & Rice & $\mathrm{MF}+\mathrm{BC}$ & HK1_Apo; BC8 & $\mathrm{S}$ & 50 & 8.0 & 3.30 & 1.18 & n.s. & 0.50 & 20.63 \\
\hline & Rice & $\mathrm{MF}+\mathrm{BC}$ & HK1_Apo; BC16 & S & 50 & 16.0 & 2.70 & 0.96 & n.s. & -0.10 & -2.06 \\
\hline & Rice & C & HK1_Vieng & $\mathrm{S}$ & 0 & & 0.70 & & & & \\
\hline & Rice & $\mathrm{BC}$ & HK1_Vieng; BC4 & S & 0 & 4.0 & 0.50 & 0.71 & n.s. & -0.20 & -16.50 \\
\hline & Rice & $\mathrm{BC}$ & HK1_Vieng; BC8 & S & 0 & 8.0 & 0.80 & 1.14 & n.s. & 0.10 & 4.13 \\
\hline & Rice & $\mathrm{BC}$ & HK1_Vieng; BC16 & S & 0 & 16.0 & 0.70 & 1.00 & n.s. & 0.00 & 0.00 \\
\hline & Rice & MF & HK1_Vieng & S & 50 & & 0.70 & & & & \\
\hline & Rice & $\mathrm{MF}+\mathrm{BC}$ & HK1_Vieng; BC4 & S & 50 & 4.0 & 1.50 & 2.14 & $*$ & 0.80 & 66.00 \\
\hline & Rice & $\mathrm{MF}+\mathrm{BC}$ & HK1_Vieng; BC8 & $S$ & 50 & 8.0 & 1.00 & 1.43 & n.s. & 0.30 & 12.38 \\
\hline & Rice & $\mathrm{MF}+\mathrm{BC}$ & HK1_Vieng; BC16 & $S$ & 50 & 16.0 & 0.80 & 1.14 & n.s. & 0.10 & 2.06 \\
\hline & Rice & $\mathrm{C}$ & LO1_Apo & S & 0 & & 5.70 & & & & \\
\hline & Rice & $\mathrm{BC}$ & LO1_Apo; BC4 & S & 0 & 4.0 & 5.10 & 0.89 & n.s. & -0.60 & -49.50 \\
\hline & Rice & $\mathrm{BC}$ & LO1_Apo; BC8 & S & 0 & 8.0 & 5.60 & 0.98 & n.s. & -0.10 & -4.13 \\
\hline & Rice & $\mathrm{BC}$ & LO1_Apo; BC16 & S & 0 & 16.0 & 5.90 & 1.04 & n.s. & 0.20 & 4.13 \\
\hline & Rice & MF & LO1_Apo & $S$ & 50 & & 5.40 & & & & \\
\hline & Rice & $\mathrm{MF}+\mathrm{BC}$ & LO1_Apo; BC4 & S & 50 & 4.0 & 4.80 & 0.89 & n.s. & -0.60 & -49.50 \\
\hline & Rice & $\mathrm{MF}+\mathrm{BC}$ & LO1_Apo; BC8 & S & 50 & 8.0 & 5.60 & 1.04 & n.s. & 0.20 & 8.25 \\
\hline & Rice & $\mathrm{MF}+\mathrm{BC}$ & LO1_Apo; BC16 & $S$ & 50 & 16.0 & 5.30 & 0.98 & n.s. & -0.10 & -2.06 \\
\hline & Rice & $\mathrm{C}$ & LO1_Vieng & S & 0 & & 3.80 & & & & \\
\hline & Rice & $\mathrm{BC}$ & LO1_Vieng; BC4 & $S$ & 0 & 4.0 & 3.60 & 0.95 & n.s. & -0.20 & -16.50 \\
\hline & Rice & $\mathrm{BC}$ & LO1_Vieng; BC8 & $S$ & 0 & 8.0 & 4.00 & 1.05 & n.s. & 0.20 & 8.25 \\
\hline & Rice & $\mathrm{BC}$ & LO1_Vieng; BC16 & $S$ & 0 & 16.0 & 3.70 & 0.97 & n.s. & -0.10 & -2.06 \\
\hline & Rice & MF & LO1_Vieng & S & 50 & & 3.00 & & & & \\
\hline & Rice & $\mathrm{MF}+\mathrm{BC}$ & LO1_Vieng; BC4 & S & 50 & 4.0 & 3.40 & 1.13 & n.s. & 0.40 & 33.00 \\
\hline & Rice & $\mathrm{MF}+\mathrm{BC}$ & LO1_Vieng; BC8 & S & 50 & 8.0 & 3.70 & 1.23 & n.s. & 0.70 & 28.88 \\
\hline & Rice & $\mathrm{MF}+\mathrm{BC}$ & LO1_Vieng; BC16 & $S$ & 50 & 16.0 & 3.80 & 1.27 & n.s. & 0.80 & 16.50 \\
\hline & Rice & $\mathrm{C}$ & HK2_Apo & S & 0 & & 4.80 & & & & \\
\hline & Rice & $\mathrm{BC}$ & HK2_Apo; BC8 & S & 0 & 8.0 & 4.30 & 0.90 & n.s. & -0.50 & -20.63 \\
\hline
\end{tabular}




\begin{tabular}{|c|c|c|c|c|c|c|c|c|c|c|c|}
\hline $\begin{array}{l}\text { Ref. } \\
\text { No. }\end{array}$ & Crop & Variant $^{\mathrm{a}}$ & $\begin{array}{l}\text { Treatment, plot, site, } \\
\text { year }\end{array}$ & \begin{tabular}{|c|} 
Single/ \\
Multiple \\
(year) $^{b}$
\end{tabular} & $\begin{array}{c}\mathbf{N} \text { ferti- } \\
\text { lizing } \\
(\mathrm{kg} \text { N/ha) }\end{array}$ & $\begin{array}{c}\text { Biochar } \\
\text { added } \\
\text { (Mg/ha) }\end{array}$ & $\begin{array}{c}\text { Yield } \\
\text { (Mg/ha) }\end{array}$ & $\begin{array}{l}\text { Rel. } \\
\text { Yield }\end{array}$ & $\begin{array}{l}\text { Signifi- } \\
\text { cance }^{d}\end{array}$ & $\begin{array}{c}\text { Yield } \\
\text { change }^{\mathrm{e}} \\
\text { (Mg/ha) }\end{array}$ & $\begin{array}{c}\text { Revenue }^{\text {e }} \\
\text { (USD/ } \\
\text { Mg BC) }\end{array}$ \\
\hline \multirow{12}{*}{$\begin{array}{c}\mathbf{4} \\
\text { conttd }\end{array}$} & Rice & $\mathrm{C}$ & LO2_Apo & $\mathrm{S}$ & 0 & & 4.20 & & & & \\
\hline & Rice & $\mathrm{BC}$ & LO2_Apo; BC8 & S & 0 & 8.0 & 4.70 & 1.12 & $*$ & 0.50 & 20.63 \\
\hline & Rice & $\mathrm{C}$ & HK3_Apo & S & 0 & & 1.80 & & & & \\
\hline & Rice & $\mathrm{BC}$ & HK3_Apo; BC8 & S & 0 & 8.0 & 2.20 & 1.22 & $*$ & 0.40 & 16.50 \\
\hline & Rice & C & LO3_Apo & S & 0 & & 2.40 & & & & \\
\hline & Rice & $\mathrm{BC}$ & LO3_Apo; BC8 & S & 0 & 8.0 & 2.70 & 1.13 & n.s. & 0.30 & 12.38 \\
\hline & Rice & $\mathrm{C}$ & SO-Apo & S & 0 & & 1.90 & & & & \\
\hline & Rice & $\mathrm{BC}$ & SO-Apo; BC8 & $S$ & 0 & 8.0 & 1.70 & 0.89 & n.s. & -0.20 & -8.25 \\
\hline & Rice & C & SN_Apo & S & 0 & & 3.20 & & & & \\
\hline & Rice & $\mathrm{BC}$ & SN_Apo; BC8 & $S$ & 0 & 8.0 & 3.50 & 1.09 & n.s. & 0.30 & 12.38 \\
\hline & Rice & $\mathrm{C}$ & LS_Apo & S & 0 & & 2.20 & & & & \\
\hline & Rice & $\mathrm{BC}$ & LS_Apo; BC8 & $\mathrm{S}$ & 0 & 8.0 & 1.80 & 0.82 & n.s. & -0.40 & -16.50 \\
\hline \multirow[t]{5}{*}{5} & Durum wheat & MF & & $S$ & 114 & & 2.8 & & & & \\
\hline & Durum wheat & $\mathrm{MF}+\mathrm{BC}$ & BC10 & $S$ & 114 & 10 & 3.1 & 1.11 & n.s. & 0.30 & 2.84 \\
\hline & Maize & MF & & S & 413 & & 9.7 & & & & \\
\hline & Maize & $\mathrm{MF}+\mathrm{BC}$ & $\mathrm{BC} 10(\mathrm{CR})$ & $\mathrm{S}$ & 413 & 10 & 12.1 & 1.25 & $*$ & 2.40 & 14.20 \\
\hline & Maize & $\mathrm{MF}+\mathrm{BC}$ & $\mathrm{BC} 10(\mathrm{C}+)$ & $S$ & 413 & 10 & 10.3 & 1.06 & n.s. & 0.60 & 3.55 \\
\hline \multirow[t]{4}{*}{6} & Banana & $\mathrm{MF}$ & & $S$ & 400 & & 33.19 & & & & \\
\hline & Banana & $\mathrm{MF}+\mathrm{BC}$ & & S & 400 & 10.0 & 27.06 & 0.82 & n.s. & -6.13 & \\
\hline & Banana & Compost & & S & 400 & & 28.96 & & & & \\
\hline & Banana & COMBI & & $\mathrm{S}$ & 400 & 10.0 & 25.43 & 0.88 & n.s. & -3.53 & \\
\hline \multirow{8}{*}{$\begin{array}{c}7 \\
\text { (f) }\end{array}$} & Wheat & $\mathrm{C}$ & Walkaway & $\bar{S}$ & 0 & & 1.76 & & & & \\
\hline & Wheat & $\mathrm{BC}$ & Walkaway & S & 0 & 0.1 & 1.60 & 0.91 & n.s. & -0.16 & -216.54 \\
\hline & Wheat & $\mathrm{BC}$ & Walkaway & $S$ & 0 & 0.3 & 1.87 & 1.06 & n.s. & 0.11 & 41.68 \\
\hline & Wheat & $\mathrm{BC}$ & Walkaway & S & 0 & 0.5 & 1.84 & 1.05 & n.s. & 0.08 & 15.16 \\
\hline & Wheat & $\mathrm{BC}$ & Walkaway & S & 0 & 1.0 & 1.85 & 1.05 & n.s. & 0.09 & 8.53 \\
\hline & Wheat & $\mathrm{BC}$ & Walkaway & $S$ & 0 & 2.0 & 2.14 & 1.22 & $*$ & 0.38 & 18.00 \\
\hline & Wheat & BC & Walkaway & $S$ & 0 & 3.3 & 2.02 & 1.15 & $*$ & 0.26 & 7.40 \\
\hline & Wheat & MF & Walkaway; MF50 & $S$ & 50 & & 1.84 & & & & \\
\hline
\end{tabular}




\begin{tabular}{|c|c|c|c|c|c|c|c|c|c|c|c|}
\hline $\begin{array}{l}\text { Ref. } \\
\text { No. }\end{array}$ & Crop & Variant $^{\mathrm{a}}$ & $\begin{array}{l}\text { Treatment, plot, site, } \\
\text { year }\end{array}$ & 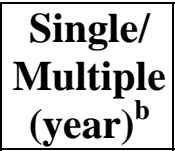 & $\begin{array}{c}\text { N ferti- } \\
\text { lizing }^{c} \\
(\text { kg N/ha) }\end{array}$ & $\begin{array}{c}\text { Biochar } \\
\text { added } \\
\text { (Mg/ha) }\end{array}$ & $\begin{array}{c}\text { Yield } \\
\text { (Mg/ha) }\end{array}$ & $\begin{array}{l}\text { Rel. } \\
\text { Yield }\end{array}$ & $\begin{array}{l}\text { Signifi- } \\
\text { cance }^{d}\end{array}$ & $\begin{array}{c}\text { Yield } \\
\text { change }^{\mathrm{e}} \\
(\mathrm{Mg} / \mathrm{ha})\end{array}$ & $\begin{array}{c}\text { Revenue }^{\mathrm{e}} \\
\text { (USD/ } \\
\text { Mg BC) }\end{array}$ \\
\hline \multirow{29}{*}{$\begin{array}{c}7 \\
\text { cont'd }^{6}\end{array}$} & Wheat & $\mathrm{BC}+\mathrm{MF}$ & Walkaway; MF50 & $\mathrm{S}$ & 50 & 0.1 & 1.71 & 0.93 & n.s. & -0.13 & -175.94 \\
\hline & Wheat & $\mathrm{BC}+\mathrm{MF}$ & Walkaway; MF50 & $\mathrm{S}$ & 50 & 0.3 & 2.04 & 1.11 & n.s. & 0.20 & 75.79 \\
\hline & Wheat & $\mathrm{BC}+\mathrm{MF}$ & Walkaway; MF50 & $\mathrm{S}$ & 50 & 0.5 & 2.10 & 1.14 & $*$ & 0.26 & 49.26 \\
\hline & Wheat & $\mathrm{BC}+\mathrm{MF}$ & Walkaway; MF50 & $\mathrm{S}$ & 50 & 1.0 & 2.16 & 1.17 & $*$ & 0.32 & 30.32 \\
\hline & Wheat & $\mathrm{BC}+\mathrm{MF}$ & Walkaway; MF50 & $\mathrm{S}$ & 50 & 2.0 & 2.27 & 1.23 & $*$ & 0.43 & 20.37 \\
\hline & Wheat & $\mathrm{BC}+\mathrm{MF}$ & Walkaway; MF50 & $\mathrm{S}$ & 50 & 3.3 & 1.95 & 1.06 & n.s. & 0.11 & 3.13 \\
\hline & Wheat & MF & Walkaway; MF100 & $\mathrm{S}$ & 0 & & 2.14 & & & & \\
\hline & Wheat & $\mathrm{BC}+\mathrm{MF}$ & Walkaway; MF100 & $\mathrm{S}$ & 100 & 0.1 & 1.92 & 0.90 & n.s. & -0.22 & -297.74 \\
\hline & Wheat & $\mathrm{BC}+\mathrm{MF}$ & Walkaway; MF100 & $\mathrm{S}$ & 100 & 0.3 & 1.99 & 0.93 & n.s. & -0.15 & -56.84 \\
\hline & Wheat & $\mathrm{BC}+\mathrm{MF}$ & Walkaway; MF100 & $\mathrm{S}$ & 100 & 0.5 & 2.20 & 1.03 & n.s. & 0.06 & 11.37 \\
\hline & Wheat & $\mathrm{BC}+\mathrm{MF}$ & Walkaway; MF100 & $\mathrm{S}$ & 100 & 1.0 & 2.18 & 1.02 & n.s. & 0.04 & 3.79 \\
\hline & Wheat & $\mathrm{BC}+\mathrm{MF}$ & Walkaway; MF100 & $\mathrm{S}$ & 100 & 2.0 & 2.25 & 1.05 & n.s. & 0.11 & 5.21 \\
\hline & Wheat & $\mathrm{BC}+\mathrm{MF}$ & Walkaway; MF100 & $\mathrm{S}$ & 100 & 3.3 & 2.02 & 0.94 & n.s. & -0.12 & -3.41 \\
\hline & Wheat & $\mathrm{C}$ & Moora & S & 0 & & 0.37 & & & & \\
\hline & Wheat & $\mathrm{BC}$ & Moora & $\mathrm{S}$ & 0 & 1.0 & 0.33 & 0.90 & n.s. & -0.04 & -3.32 \\
\hline & Wheat & MF & Moora; soluble P7 & $\mathrm{S}$ & 0 & & 0.57 & & & & \\
\hline & Wheat & $\mathrm{MF}+\mathrm{BC}$ & Moora; soluble P7 & $\mathrm{S}$ & 0 & 1.0 & 0.80 & 1.40 & $*$ & 0.23 & 21.51 \\
\hline & Wheat & MF & Moora; soluble P20 & $\mathrm{S}$ & 0 & & 0.76 & & & & \\
\hline & Wheat & $\mathrm{MF}+\mathrm{BC}$ & Moora; soluble P20 & $\mathrm{S}$ & 0 & 1.0 & 0.87 & 1.15 & $*$ & 0.11 & 10.61 \\
\hline & Wheat & $\mathrm{MF}$ & Moora; rock P7 & S & 0 & & 0.38 & & & & \\
\hline & Wheat & $\mathrm{MF}+\mathrm{BC}$ & Moora; rock P7 & $\mathrm{S}$ & 0 & 1.0 & 0.37 & 0.99 & n.s. & 0.00 & -0.38 \\
\hline & Wheat & MF & Moora; P- & $\mathrm{S}$ & 0 & & 0.38 & & & & \\
\hline & Wheat & $\mathrm{MF}+\mathrm{BC}$ & Moora; P- & $\mathrm{S}$ & 0 & 1.0 & 0.43 & 1.16 & n.s. & 0.06 & 5.59 \\
\hline & Wheat & $\mathrm{MF}+\mathrm{Mic}$ & Moora; P+; Mic & $\mathrm{S}$ & 0 & & 0.44 & & & & \\
\hline & Wheat & $\mathrm{MF}+\mathrm{BC}+\mathrm{Mic}$ & Moora; P+; Mic & $\mathrm{S}$ & 0 & 1.0 & 0.39 & 0.87 & n.s. & -0.06 & -5.31 \\
\hline & Wheat & MF & Moora & S & 0 & & 0.72 & & & & \\
\hline & Wheat & $\mathrm{BC}+\mathrm{MF}$ & Moora & $\mathrm{S}$ & 0 & 1.0 & 0.74 & 1.02 & n.s. & 0.02 & 1.71 \\
\hline & Wheat & $\mathrm{MF}$ & Shea-Oak Log, DAP & $\mathrm{S}$ & 0 & & 2.72 & & & & \\
\hline & Wheat & $\mathrm{MF}+\mathrm{BC}$ & Shea-Oak Log; DAP & $\mathrm{S}$ & 0 & 0.8 & 2.59 & 0.95 & n.s. & -0.13 & -16.42 \\
\hline
\end{tabular}




\begin{tabular}{|c|c|c|c|c|c|c|c|c|c|c|c|}
\hline $\begin{array}{l}\text { Ref. } \\
\text { No. }\end{array}$ & Crop & Variant $^{\mathrm{a}}$ & $\begin{array}{l}\text { Treatment, plot, site, } \\
\text { year }\end{array}$ & $\begin{array}{c}\text { Single/ } \\
\text { Multiple } \\
\text { (year) }^{b}\end{array}$ & $\begin{array}{c}\text { N ferti- } \\
\text { lizing }^{c} \\
\text { (kg N/ha) }\end{array}$ & $\begin{array}{c}\text { Biochar } \\
\text { added } \\
\text { (Mg/ha) }\end{array}$ & $\begin{array}{c}\text { Yield } \\
\text { (Mg/ha) }\end{array}$ & $\begin{array}{l}\text { Rel. } \\
\text { Yield }\end{array}$ & $\begin{array}{l}\text { Signifi- } \\
\text { cance }^{d}\end{array}$ & $\begin{array}{c}\text { Yield } \\
\text { change }^{\mathrm{e}} \\
(\mathrm{Mg} / \mathrm{ha})\end{array}$ & $\begin{array}{c}\text { Revenue }^{\mathrm{e}} \\
\text { (USD/ } \\
\text { Mg BC) }\end{array}$ \\
\hline \multirow{2}{*}{$\begin{array}{c}7 \\
\text { cont }^{6} \mathrm{~d}\end{array}$} & Wheat & $\mathrm{MF}$ & Templers; DAP & $\mathrm{S}$ & 0 & & 3.33 & & & & \\
\hline & Wheat & $\mathrm{MF}+\mathrm{BC}$ & Templers; DAP & S & 0 & 0.8 & 3.33 & 1.00 & n.s. & 0.00 & 0.00 \\
\hline \multirow{6}{*}{$\begin{array}{c}8 \\
(g)\end{array}$} & Maize & $\mathrm{C}$ & 2008 & Y1 & yes & & 8.56 & & & & \\
\hline & Maize & BC45 & 2008 & Y1 & yes & 45.0 & 4.49 & 0.52 & $*$ & -4.07 & -5.35 \\
\hline & Maize & $\mathrm{C}$ & 2009 & Y2 & yes & & 12.93 & & & & \\
\hline & Maize & BC45 & 2009 & Y2 & yes & 0 & 6.59 & 0.51 & $*$ & -6.34 & -8.34 \\
\hline & Maize & $\mathrm{C}$ & 2010 & Y3 & yes & & 1.13 & & & & \\
\hline & Maize & BC45 & 2010 & Y3 & yes & 0 & 1.34 & 1.19 & n.s. & 0.21 & 0.28 \\
\hline \multirow{21}{*}{$\begin{array}{c}9 \\
(\mathrm{~h})\end{array}$} & Maize & $\mathrm{MF}$ & Kaomo (2011) & Y1 & 77 & & 0.8 & & & & \\
\hline & Maize & $\mathrm{MF}+\mathrm{BC} 0.8($ maize $)$ & Kaomo (2011) & Y1 & 77 & 6.0 & 1.0 & 1.25 & n.s. & 0.20 & 1.97 \\
\hline & Maize & MF+BC4(maize) & Kaomo (2011) & Y1 & 77 & 30.0 & 4.2 & 5.25 & $*$ & 3.40 & 6.71 \\
\hline & Maize & $\mathrm{MF}+\mathrm{BC} 0.8($ wood $)$ & Kaomo (2011) & Y1 & 77 & 6.0 & 1.2 & 1.50 & n.s. & 0.40 & 3.94 \\
\hline & Maize & MF+BC4(wood) & Kaomo (2011) & Y1 & 77 & 30.0 & 3.6 & 4.50 & $*$ & 2.80 & 5.52 \\
\hline & Maize & $\mathrm{MF}$ & Kaomo (2012) & Y2 & 77 & & 0.96 & & & & \\
\hline & Maize & $\mathrm{MF}+\mathrm{BC} 0.8($ maize$)$ & Kaomo (2012) & Y2 & 77 & 0 & 1.32 & 1.38 & n.s. & 0.36 & 3.55 \\
\hline & Maize & MF+BC4(maize) & Kaomo (2012) & Y2 & 77 & 0 & 3.49 & 3.64 & $*$ & 2.53 & 4.99 \\
\hline & Maize & $\mathrm{MF}+\mathrm{BC} 0.8($ wood $)$ & Kaomo (2012) & Y2 & 77 & 0 & 1.13 & 1.18 & n.s. & 0.17 & 1.68 \\
\hline & Maize & $\mathrm{MF}+\mathrm{BC} 4($ wood) & Kaomo (2012) & Y2 & 77 & 0 & 2.43 & 2.53 & $*$ & 1.47 & 2.90 \\
\hline & Maize & MF & UNZA (2011) & S & 154 & & 2.6 & & & & \\
\hline & Maize & MF+BC5(maize) & UNZA (2011) & S & 154 & 30.0 & 3.7 & 1.42 & n.s. & 1.10 & 2.17 \\
\hline & Maize & $\mathrm{MF}+\mathrm{BC} 1$ (wood) & UNZA (2011) & $\mathrm{S}$ & 154 & 6.0 & 2.8 & 1.08 & n.s. & 0.20 & 1.97 \\
\hline & Maize & $\mathrm{MF}+\mathrm{BC} 5$ (wood) & UNZA (2011) & $\mathrm{S}$ & 154 & 30.0 & 3.4 & 1.31 & n.s. & 0.80 & 1.58 \\
\hline & Maize & $\mathrm{MF}$ & Mkushi (2011) & Y1 & 77 & & 4.8 & & & & \\
\hline & Maize & $\mathrm{MF}+\mathrm{BC} 0.8$ (maize) & Mkushi (2011) & Y1 & 77 & 6.0 & 4.2 & 0.88 & n.s. & -0.60 & -5.92 \\
\hline & Maize & MF+BC4(maize) & Mkushi (2011) & Y1 & 77 & 30.0 & 3.5 & 0.73 & n.s. & -1.30 & -2.56 \\
\hline & Maize & $\mathrm{MF}+\mathrm{BC} 0.8($ wood $)$ & Mkushi (2011) & Y1 & 77 & 6.0 & 4.5 & 0.94 & n.s. & -0.30 & -2.96 \\
\hline & Maize & MF+BC4(wood) & Mkushi (2011) & Y1 & 77 & 30.0 & 5.6 & 1.17 & n.s. & 0.80 & 1.58 \\
\hline & Maize & MF & Mkushi (2012) & Y2 & 77 & & 5.84 & & & & \\
\hline & Maize & $\mathrm{MF}+\mathrm{BC} 0.8$ (maize) & Mkushi (2012) & Y2 & 77 & 0 & 6.32 & 1.08 & n.s. & 0.48 & 4.73 \\
\hline
\end{tabular}




\begin{tabular}{|c|c|c|c|c|c|c|c|c|c|c|c|}
\hline $\begin{array}{l}\text { Ref. } \\
\text { No. }\end{array}$ & Crop & Variant $^{\mathrm{a}}$ & $\begin{array}{l}\text { Treatment, plot, site, } \\
\text { year }\end{array}$ & $\begin{array}{c}\text { Single/ } \\
\text { Multiple } \\
\text { (year) }^{b}\end{array}$ & $\begin{array}{c}\text { N ferti- } \\
\text { lizing } \\
(\mathrm{kg} N / h a)\end{array}$ & $\begin{array}{c}\text { Biochar } \\
\text { added } \\
\text { (Mg/ha) }\end{array}$ & $\begin{array}{c}\text { Yield } \\
\text { (Mg/ha) }\end{array}$ & $\begin{array}{l}\text { Rel. } \\
\text { Yield }\end{array}$ & $\begin{array}{l}\text { Signifi- }^{\text {cance }^{d}} \\
\end{array}$ & $\begin{array}{c}\text { Yield } \\
\text { change }^{\mathrm{e}} \\
(\mathrm{Mg} / \mathrm{ha})\end{array}$ & $\begin{array}{c}\text { Revenue }^{\mathrm{e}} \\
\text { (USD/ } \\
\text { Mg BC) }\end{array}$ \\
\hline \multirow{13}{*}{$\begin{array}{c}9 \\
\text { cont }^{6} \mathrm{~d}\end{array}$} & Maize & MF+BC4(maize) & Mkushi (2012) & $\mathrm{Y} 2$ & 77 & 0 & 4.80 & 0.82 & n.s. & -1.04 & -2.05 \\
\hline & Maize & $\mathrm{MF}+\mathrm{BC} 0.8($ wood $)$ & Mkushi (2012) & Y2 & 77 & 0 & 5.84 & 1.00 & n.s. & 0.00 & 0.00 \\
\hline & Maize & MF+BC4(wood) & Mkushi (2012) & Y2 & 77 & 0 & 6.4 & 1.10 & n.s. & 0.56 & 1.10 \\
\hline & Maize & $\mathrm{MF}$ & Lusaka (2011) & S & 154 & & 9.1 & & & & \\
\hline & Maize & $\mathrm{MF}+\mathrm{BC} 0.8$ (maize) & Lusaka (2011) & S & 154 & 6.0 & 9.0 & 0.99 & n.s. & -0.10 & -0.99 \\
\hline & Maize & MF+BC4(maize) & Lusaka (2011) & $\mathrm{S}$ & 154 & 30.0 & 8.2 & 0.90 & n.s. & -0.90 & -1.78 \\
\hline & Maize & $\mathrm{MF}+\mathrm{BC} 0.8($ wood $)$ & Lusaka (2011) & $S$ & 154 & 6.0 & 6.8 & 0.75 & n.s. & -2.30 & -22.68 \\
\hline & Maize & MF+BC4(wood) & Lusaka (2011) & S & 154 & 30.0 & 6.9 & 0.76 & n.s. & -2.20 & -4.34 \\
\hline & Maize & $\mathrm{MF}$ & Shim (2011) & S & 154 & & 8.6 & & & & \\
\hline & Maize & $\mathrm{MF}+\mathrm{BC} 0.8$ (maize) & Shim (2011) & S & 154 & 6.0 & 11.0 & 1.28 & n.s. & 2.40 & 23.67 \\
\hline & Maize & MF+BC4(maize) & Shim (2011) & $S$ & 154 & 30.0 & 6.7 & 0.78 & n.s. & -1.90 & -3.75 \\
\hline & Maize & $\mathrm{MF}+\mathrm{BC} 0.8($ wood $)$ & Shim (2011) & $\mathrm{S}$ & 154 & 6.0 & 8.1 & 0.94 & n.s. & -0.50 & -4.93 \\
\hline & Maize & $\mathrm{MF}+\mathrm{BC} 4($ wood $)$ & Shim (2011) & $\mathrm{S}$ & 154 & 30.0 & 8.1 & 0.94 & n.s. & -0.50 & -0.99 \\
\hline \multirow[t]{8}{*}{10} & Wheat & $\mathrm{C}$ & Year 1 & Y1 & 120 & & 2.45 & & & & \\
\hline & Wheat & $\mathrm{MF}+\mathrm{BC}$ & Year 1 & Y1 & 120 & 10 & 2.00 & 0.82 & n.s. & -0.45 & -4.26 \\
\hline & Wheat & $\mathrm{MF}+\mathrm{BC}$ & Year 1 & Y1 & 120 & 20 & 2.49 & 1.02 & n.s. & 0.04 & 0.19 \\
\hline & Wheat & $\mathrm{MF}+\mathrm{BC}$ & Year 1 & Y1 & 120 & 40 & 2.53 & 1.03 & n.s. & 0.08 & 0.19 \\
\hline & Wheat & $\mathrm{C}$ & Year 2 & Y2 & 120 & & 5.31 & & & & \\
\hline & Wheat & $\mathrm{MF}+\mathrm{BC}$ & Year 2 & Y2 & 120 & 0 & 5.62 & 1.06 & n.s. & 0.31 & 2.94 \\
\hline & Wheat & $\mathrm{MF}+\mathrm{BC}$ & Year 2 & Y2 & 120 & 0 & 5.66 & 1.07 & n.s. & 0.35 & 1.66 \\
\hline & Wheat & $\mathrm{MF}+\mathrm{BC}$ & Year 2 & Y2 & 120 & 0 & 5.91 & 1.11 & n.s. & 0.60 & 1.42 \\
\hline \multirow[t]{8}{*}{11} & Barley & $\mathrm{MF}$ & Inceptosol & S & 70 & & 27.1 & & & & \\
\hline & Barley & $\mathrm{MF}+\mathrm{BC} 5$ & Inceptosol & $S$ & 70 & 5.0 & 23.8 & 0.88 & n.s. & -3.30 & -55.79 \\
\hline & Barley & $\mathrm{MF}+\mathrm{BC} 10$ & Inceptosol & $\mathrm{S}$ & 70 & 10.0 & 25.3 & 0.93 & n.s. & -1.80 & -15.22 \\
\hline & Barley & $\mathrm{MF}+\mathrm{BC} 20$ & Inceptosol & S & 70 & 20.0 & 28.5 & 1.05 & $*$ & 1.40 & 5.92 \\
\hline & Barley & $\mathrm{MF}$ & Ultisol & S & 60 & & 2.35 & & & & \\
\hline & Barley & $\mathrm{MF}+\mathrm{BC} 5$ & Ultisol & $\mathrm{S}$ & 60 & 5.0 & 2.59 & 1.10 & n.s. & 0.24 & 4.06 \\
\hline & Barley & $\mathrm{MF}+\mathrm{BC} 10$ & Ultisol & $\mathrm{S}$ & 60 & 10.0 & 2.75 & 1.17 & n.s. & 0.40 & 3.38 \\
\hline & Barley & $\mathrm{MF}+\mathrm{BC} 20$ & Ultisol & $\mathrm{S}$ & 60 & 20.0 & 2.87 & 1.22 & $*$ & 0.52 & 2.20 \\
\hline
\end{tabular}




\begin{tabular}{|c|c|c|c|c|c|c|c|c|c|c|c|}
\hline $\begin{array}{l}\text { Ref. } \\
\text { No. }\end{array}$ & Crop & Variant $^{\mathrm{a}}$ & $\begin{array}{l}\text { Treatment, plot, site, } \\
\text { year }\end{array}$ & $\begin{array}{c}\text { Single/ } \\
\text { Multiple } \\
\text { (year) }^{b}\end{array}$ & $\begin{array}{c}\text { N ferti- } \\
\text { lizing }^{c} \\
\text { (kg N/ha) }\end{array}$ & $\begin{array}{c}\text { Biochar } \\
\text { added } \\
\text { (Mg/ha) }\end{array}$ & $\begin{array}{c}\text { Yield } \\
\text { (Mg/ha) }\end{array}$ & $\begin{array}{l}\text { Rel. } \\
\text { Yield }\end{array}$ & $\begin{array}{l}\text { Signifi- } \\
\text { cance }^{d}\end{array}$ & $\begin{array}{c}\text { Yield } \\
\text { change }^{\mathrm{e}} \\
\text { (Mg/ha) }\end{array}$ & $\begin{array}{c}\text { Revenue }^{\mathrm{e}} \\
\text { (USD/ } \\
\text { Mg BC) }\end{array}$ \\
\hline \multirow[t]{24}{*}{12} & Wheat & $\mathrm{C}$ & 2010 & Y1 & 0 & & 2.48 & & & & \\
\hline & Wheat & BC0.04 & 2010 & Y1 & 0 & 0.035 & 2.33 & 0.94 & n.s. & -0.15 & -407.34 \\
\hline & Wheat & BC 0.18 & 2010 & Y1 & 0 & 0.175 & 2.36 & 0.95 & n.s. & -0.13 & -68.43 \\
\hline & Wheat & BC5 & 2010 & Y1 & 0 & 5.0 & 2.38 & 0.96 & & -0.10 & -1.96 \\
\hline & Wheat & MF(DAP low) & 2010 & Y1 & 6 & & 2.63 & & & & \\
\hline & Wheat & $\mathrm{MF}($ low $)+\mathrm{BC}$ & 2010 & Y1 & 6 & 0.035 & 2.60 & 0.99 & n.s. & -0.02 & -65.17 \\
\hline & Wheat & $\mathrm{MF}($ low $)+\mathrm{BC}$ & 2010 & Y1 & 6 & 0.175 & 2.55 & 0.97 & n.s. & -0.08 & -43.45 \\
\hline & Wheat & $\mathrm{MF}($ low $)+\mathrm{BC}$ & 2010 & Y1 & 6 & 5.0 & 2.63 & 1.00 & & 0.01 & 0.11 \\
\hline & Wheat & MF(DAP high) & 2010 & Y1 & 13 & & 2.68 & & & & \\
\hline & Wheat & $\mathrm{MF}($ high $)+\mathrm{BC}$ & 2010 & Y1 & 13 & 0.035 & 2.72 & 1.01 & n.s. & 0.04 & 103.19 \\
\hline & Wheat & $\mathrm{MF}($ high $)+\mathrm{BC}$ & 2010 & Y1 & 13 & 0.175 & 2.73 & 1.02 & n.s. & 0.04 & 23.90 \\
\hline & Wheat & $\mathrm{MF}($ high $)+\mathrm{BC}$ & 2010 & Y1 & 13 & 5.0 & 2.87 & 1.07 & & 0.18 & 3.48 \\
\hline & Wheat & $\mathrm{C}$ & 2011 & Y2 & 0 & & 2.07 & & & & \\
\hline & Wheat & $\mathrm{BC}$ & 2011 & Y2 & 0 & 0 & 1.70 & 0.82 & n.s. & -0.37 & -1001.50 \\
\hline & Wheat & $\mathrm{BC}$ & 2011 & Y2 & 0 & 0 & 1.72 & 0.83 & n.s. & -0.35 & -189.47 \\
\hline & Wheat & $\mathrm{BC}$ & 2011 & Y2 & 0 & 0 & 1.85 & 0.89 & & -0.22 & -4.17 \\
\hline & Wheat & MF(DAP low) & 2011 & Y2 & 6 & & 2.01 & & & & \\
\hline & Wheat & $\mathrm{MF}($ low $)+\mathrm{BC}$ & 2011 & Y2 & 6 & 0 & 2.05 & 1.02 & n.s. & 0.04 & 108.27 \\
\hline & Wheat & $\mathrm{MF}($ low $)+\mathrm{BC}$ & 2011 & Y2 & 6 & 0 & 1.83 & 0.91 & n.s. & -0.18 & -95.64 \\
\hline & Wheat & $\mathrm{MF}($ low $)+\mathrm{BC}$ & 2011 & Y2 & 6 & 0 & 2.04 & 1.01 & & 0.03 & 0.51 \\
\hline & Wheat & MF(DAP high) & 2011 & Y2 & 13 & & 2.05 & & & & \\
\hline & Wheat & $\mathrm{MF}($ high$)+\mathrm{BC}$ & 2011 & Y2 & 13 & 0 & 2.22 & 1.08 & n.s. & 0.17 & 451.13 \\
\hline & Wheat & $\mathrm{MF}($ high $)+\mathrm{BC}$ & 2011 & Y2 & 13 & 0 & 2.03 & 0.99 & n.s. & -0.02 & -10.83 \\
\hline & Wheat & $\mathrm{MF}$ (high)+BC & 2011 & $\mathrm{Y} 2$ & 13 & 0 & 2.25 & 1.10 & n.s. & 0.20 & 3.79 \\
\hline \multirow[t]{5}{*}{13} & Sugar beet & $\mathrm{C}$ & & $\mathrm{S}$ & 0 & & 40.3 & & & & \\
\hline & Sugar beet & BC(HSP) & & $\mathrm{S}$ & 0 & 10 & 10.2 & 0.25 & $*$ & -30.06 & -435.87 \\
\hline & Sugar beet & BC(HBD) & & $\mathrm{S}$ & 0 & 10 & 26.9 & 0.67 & $*$ & -13.32 & -193.14 \\
\hline & Sugar beet & $\mathrm{C}+\mathrm{MF} 50$ & & S & 50 & & 45.8 & & & & \\
\hline & Sugar beet & $\mathrm{BC}(\mathrm{HSP})+\mathrm{MF} 50$ & & $\mathrm{~S}$ & 50 & 10 & 25.0 & 0.55 & $*$ & -20.76 & -301.02 \\
\hline
\end{tabular}




\begin{tabular}{|c|c|c|c|c|c|c|c|c|c|c|c|}
\hline $\begin{array}{l}\text { Ref. } \\
\text { No. }\end{array}$ & Crop & Variant $^{\mathrm{a}}$ & $\begin{array}{l}\text { Treatment, plot, site, } \\
\text { year }\end{array}$ & $\begin{array}{c}\text { Single/ } \\
\text { Multiple } \\
\text { (year) }^{b}\end{array}$ & $\begin{array}{c}\text { N ferti- } \\
\text { lizing } \\
\text { (kg N/ha) }\end{array}$ & $\begin{array}{c}\text { Biochar } \\
\text { added } \\
\text { (Mg/ha) }\end{array}$ & $\begin{array}{c}\text { Yield } \\
\text { (Mg/ha) }\end{array}$ & $\begin{array}{l}\text { Rel. } \\
\text { Yield }\end{array}$ & $\begin{array}{l}\text { Signifi- } \\
\text { cance }^{d}\end{array}$ & $\begin{array}{c}\text { Yield } \\
\text { change }^{\mathrm{e}} \\
(\mathrm{Mg} / \mathrm{ha})\end{array}$ & $\begin{array}{c}\text { Revenue }^{\text {e }} \\
\text { (USD/ } \\
\text { Mg BC) }\end{array}$ \\
\hline \multirow{4}{*}{$\begin{array}{c}13 \\
\text { cont }^{t} \mathrm{~d}\end{array}$} & Sugar beet & $\mathrm{BC}(\mathrm{HBD})+\mathrm{MF} 50$ & & $\mathrm{~S}$ & 50 & 10 & 31.0 & 0.68 & $*$ & -14.82 & -214.89 \\
\hline & Sugar beet & C+MF100 & & S & 100 & & 49.8 & & & & \\
\hline & Sugar beet & BC(HSP)+MF100 & & S & 100 & 10 & 32.5 & 0.65 & $*$ & -17.34 & -251.43 \\
\hline & Sugar beet & $\mathrm{BC}(\mathrm{HBD})+\mathrm{MF} 100$ & & $S$ & 100 & 10 & 32.2 & 0.65 & $*$ & -17.58 & -254.91 \\
\hline \multirow[t]{24}{*}{14} & Maize & $\mathrm{C}$ & Peanut hull (2006) & $\mathrm{Y} 1$ & 26 & & 4.70 & & & & \\
\hline & Maize & $\mathrm{BC}$ & Peanut hull (2006) & Y1 & 26 & 11.0 & 3.05 & 0.65 & n.s. & -1.65 & -8.88 \\
\hline & Maize & $\mathrm{BC}$ & Peanut hull (2006) & Y1 & 26 & 22.0 & 6.07 & 1.29 & n.s. & 1.37 & 3.68 \\
\hline & Maize & MF & Peanut hull (2006) & Y1 & 192 & & 13.00 & & & & \\
\hline & Maize & $\mathrm{MB}+\mathrm{BC}$ & Peanut hull (2006) & Y1 & 192 & 11.0 & 13.42 & 1.03 & n.s. & 0.42 & 2.26 \\
\hline & Maize & $\mathrm{MB}+\mathrm{BC}$ & Peanut hull (2006) & Y1 & 192 & 22.0 & 11.68 & 0.90 & n.s. & -1.32 & -3.55 \\
\hline & Maize & C & Peanut hull (2007) & Y2 & 22 & & 0.92 & & & & \\
\hline & Maize & $\mathrm{BC}$ & Peanut hull (2007) & $\mathrm{Y} 2$ & 22 & 0 & 0.92 & 1.00 & n.s. & 0.00 & 0.00 \\
\hline & Maize & $\mathrm{BC}$ & Peanut hull (2007) & $\mathrm{Y} 2$ & 22 & 0 & 1.36 & 1.48 & n.s. & 0.44 & 1.18 \\
\hline & Maize & MF & Peanut hull (2007) & $\mathrm{Y} 2$ & 246 & & 8.61 & & & & \\
\hline & Maize & $\mathrm{MB}+\mathrm{BC}$ & Peanut hull (2007) & $\mathrm{Y} 2$ & 246 & 0 & 8.55 & 0.99 & n.s. & -0.06 & -0.32 \\
\hline & Maize & $\mathrm{MB}+\mathrm{BC}$ & Peanut hull (2007) & $\mathrm{Y} 2$ & 246 & 0 & 8.09 & 0.94 & n.s. & -0.52 & -1.40 \\
\hline & Maize & $\mathrm{C}$ & Pine chip (2006) & Y1 & 26 & & 4.45 & & & & \\
\hline & Maize & $\mathrm{BC}$ & Pine chip (2006) & Y1 & 26 & 11.0 & 3.41 & 0.77 & n.s. & -1.04 & -5.59 \\
\hline & Maize & $\mathrm{BC}$ & Pine chip (2006) & Y1 & 26 & 22.0 & 3.12 & 0.70 & n.s. & -1.33 & -3.58 \\
\hline & Maize & MF & Pine chip (2006) & Y1 & 192 & & 15.13 & & & & \\
\hline & Maize & $\mathrm{MB}+\mathrm{BC}$ & Pine chip (2006) & Y1 & 192 & 11.0 & 14.52 & 0.96 & n.s. & -0.61 & -3.28 \\
\hline & Maize & $\mathrm{MB}+\mathrm{BC}$ & Pine chip (2006) & Y1 & 192 & 22.0 & 13.65 & 0.90 & n.s. & -1.48 & -3.98 \\
\hline & Maize & $\mathrm{C}$ & Pine chip (2007) & Y2 & 22 & & 0.52 & & & & \\
\hline & Maize & $\mathrm{BC}$ & Pine chip (2007) & Y2 & 22 & 0 & 0.74 & 1.42 & n.s. & 0.22 & 1.18 \\
\hline & Maize & $\mathrm{BC}$ & Pine chip (2007) & $\mathrm{Y} 2$ & 22 & 0 & 0.81 & 1.56 & n.s. & 0.29 & 0.78 \\
\hline & Maize & MF & Pine chip (2007) & Y2 & 246 & & 8.70 & & & & \\
\hline & Maize & $\mathrm{MB}+\mathrm{BC}$ & Pine chip (2007) & $\mathrm{Y} 2$ & 246 & 0 & 10.69 & 1.23 & n.s. & 1.99 & 10.70 \\
\hline & Maize & $\mathrm{MB}+\mathrm{BC}$ & Pine chip (2007) & $\mathrm{Y} 2$ & 246 & 0 & 8.87 & 1.02 & n.s. & 0.17 & 0.46 \\
\hline
\end{tabular}




\begin{tabular}{|c|c|c|c|c|c|c|c|c|c|c|c|}
\hline $\begin{array}{l}\text { Ref. } \\
\text { No. }\end{array}$ & Crop & Variant $^{\mathrm{a}}$ & $\begin{array}{l}\text { Treatment, plot, site, } \\
\text { year }\end{array}$ & $\begin{array}{c}\text { Single/ } \\
\text { Multiple } \\
\text { (year) }^{b}\end{array}$ & $\begin{array}{c}\text { N ferti- } \\
\text { lizing } \\
\text { (kg N/ha) }\end{array}$ & $\begin{array}{c}\text { Biochar } \\
\text { added } \\
(\mathrm{Mg} / \mathrm{ha})\end{array}$ & $\begin{array}{c}\text { Yield } \\
\text { (Mg/ha) } \\
\end{array}$ & $\begin{array}{l}\text { Rel. } \\
\text { Yield }\end{array}$ & $\begin{array}{l}\text { Signifi- } \\
\text { cance }^{d}\end{array}$ & $\begin{array}{c}\text { Yield } \\
\text { change }^{\mathrm{e}} \\
(\mathrm{Mg} / \mathrm{ha})\end{array}$ & $\begin{array}{c}\text { Revenue }^{\mathrm{e}} \\
\text { (USD/ } \\
\text { Mg BC) } \\
\end{array}$ \\
\hline \multirow[t]{10}{*}{15} & Silage maize & $\mathrm{MF}$ & & $\bar{S}$ & 200 & & 5.43 & & & & \\
\hline & Silage maize & $\mathrm{MF}+\mathrm{BC}$ & & S & 200 & 1.0 & 7.03 & 1.30 & n.s. & 1.60 & \\
\hline & Silage maize & $\mathrm{MF}+\mathrm{BC}$ & & S & 200 & 40.0 & 5.50 & 1.01 & n.s. & 0.07 & \\
\hline & Silage maize & MF+Dig & & S & 200 & & 5.56 & & & & \\
\hline & Silage maize & \multirow{2}{*}{\multicolumn{2}{|c|}{$\begin{array}{l}\mathrm{MF}+\mathrm{Dig}+\mathrm{BC} \\
\mathrm{MF}+\mathrm{Dig}+\mathrm{BC}\end{array}$}} & S & 200 & 1,0 & 6.67 & 1.20 & n.s. & 1.10 & \\
\hline & Silage maize & & & $S$ & 200 & 40,0 & 7.89 & 1.42 & $*$ & 2.32 & \\
\hline & Silage maize & MF+Dig(ferm) & & S & 200 & & 8.90 & & & & \\
\hline & Silage maize & \multicolumn{2}{|l|}{$\mathrm{MF}+\operatorname{Dig}(\mathrm{f})+\mathrm{BC}$} & S & 200 & 40,0 & 4.55 & 0.51 & $*$ & -4.35 & \\
\hline & Silage maize & $\mathrm{MF}+\mathrm{Co}$ & & $\mathrm{S}$ & 200 & & 5.58 & & & & \\
\hline & Silage maize & $\mathrm{MF}+\mathrm{Co}+\mathrm{BC}$ & & S & 200 & 10.0 & 7.03 & 1.26 & & 1.45 & \\
\hline \multirow[t]{15}{*}{16} & Maize & $\mathrm{C}$ & $\mathrm{T} 1$ & $\bar{S}$ & 0 & & 2.54 & & & & \\
\hline & Maize & $\mathrm{BC}$ & $\mathrm{T} 2$ & S & 0 & 5.0 & 4.60 & 1.81 & $*$ & 2.06 & 24.38 \\
\hline & Maize & $\mathrm{BC}$ & $\mathrm{T} 3$ & S & 0 & 7.5 & 4.85 & 1.91 & $*$ & 2.31 & 18.22 \\
\hline & Maize & MF75\% & $\mathrm{T} 4$ & S & 101 & & 4.85 & & & & \\
\hline & Maize & MF75\%+BC & T8 & $\mathrm{S}$ & 101 & 5.0 & 7.15 & 1.47 & $*$ & 2.30 & 27.22 \\
\hline & Maize & MF75\%+BC & $\mathrm{T} 10$ & S & 101 & 7.5 & 7.45 & 1.54 & $*$ & 2.60 & 20.51 \\
\hline & Maize & MF100\% & T5 & S & 135 & & 5.08 & & & & \\
\hline & Maize & MF100\%+BC & T9 & S & 135 & 5.0 & 7.28 & 1.43 & $*$ & 2.20 & 26.04 \\
\hline & Maize & $\mathrm{MF} 100 \%+\mathrm{BC}$ & $\mathrm{T} 11$ & $\mathrm{~S}$ & 135 & 7.5 & 7.65 & 1.51 & $*$ & 2.57 & 20.28 \\
\hline & Maize & MF75\%+biofert & T6 & S & 101 & & 5.00 & & & & \\
\hline & Maize & MF75\%+biofert+BC & $\mathrm{T} 12$ & S & 101 & 5.0 & 7.70 & 1.54 & $*$ & 2.70 & 31.95 \\
\hline & Maize & MF75\%+biofert+BC & $\mathrm{T} 14$ & $S$ & 101 & 7.5 & 7.60 & 1.52 & * & 2.60 & 20.51 \\
\hline & Maize & MF100\%+biofert & $\mathrm{T} 7$ & S & 135 & & 5.13 & & & & \\
\hline & Maize & MF100\%+biofert+BC & $\mathrm{T} 13$ & S & 135 & 5.0 & 8.10 & 1.58 & $*$ & 2.97 & 35.15 \\
\hline & Maize & MF100\%+biofert+BC & $\mathrm{T} 15$ & $S$ & 135 & 7.5 & 7.73 & 1.51 & $*$ & 2.60 & 20.51 \\
\hline \multirow{4}{*}{$\begin{array}{l}17 \\
\text { (i) }\end{array}$} & Maize & $\mathrm{C}(90 \% \mathrm{~N})$ & Year 1 & $\overline{\mathrm{Y} 1}$ & 109 & & 4.74 & & & & \\
\hline & Maize & BC & Year 1 & Y1 & 109 & 3 & 4.11 & 0.87 & n.s. & -0.63 & -12.43 \\
\hline & Maize & $\mathrm{BC}$ & Year 1 & Y1 & 109 & 12 & 4.19 & 0.88 & n.s. & -0.55 & -2.71 \\
\hline & Maize & BC & Year 1 & Y1 & 109 & 30 & 4.02 & 0.85 & n.s. & -0.72 & -1.42 \\
\hline
\end{tabular}




\begin{tabular}{|c|c|c|c|c|c|c|c|c|c|c|c|}
\hline $\begin{array}{l}\text { Ref. } \\
\text { No. }\end{array}$ & Crop & Variant $^{\mathrm{a}}$ & $\begin{array}{l}\text { Treatment, plot, site, } \\
\text { year }\end{array}$ & $\begin{array}{c}\text { Single/ } \\
\text { Multiple } \\
\text { (year) }\end{array}$ & $\begin{array}{c}\text { N ferti- } \\
\text { lizing }^{c} \\
\text { (kg N/ha) }\end{array}$ & $\begin{array}{c}\text { Biochar } \\
\text { added } \\
\text { (Mg/ha) }\end{array}$ & $\begin{array}{c}\text { Yield } \\
\text { (Mg/ha) }\end{array}$ & $\begin{array}{l}\text { Rel. } \\
\text { Yield }\end{array}$ & $\begin{array}{l}\text { Signifi- } \\
\text { cance }^{d}\end{array}$ & $\begin{array}{c}\text { Yield } \\
\text { change }^{\mathrm{e}} \\
\text { (Mg/ha) }\end{array}$ & $\begin{array}{c}\text { Revenue }^{\mathrm{e}} \\
\text { (USD/ } \\
\text { Mg BC) }\end{array}$ \\
\hline \multirow{29}{*}{$\begin{array}{c}17 \\
\text { cont }^{6} \mathrm{~d}\end{array}$} & Maize & $\mathrm{C}(90 \% \mathrm{~N})$ & Year 2 & $\mathrm{Y} 2$ & 109 & & 9.26 & & & & \\
\hline & Maize & $\mathrm{BC}$ & Year 2 & Y2 & 109 & 0 & 7.8 & 0.84 & n.s. & -1.46 & -28.80 \\
\hline & Maize & $\mathrm{BC}$ & Year 2 & Y2 & 109 & 0 & 8.14 & 0.88 & n.s. & -1.12 & -5.52 \\
\hline & Maize & $\mathrm{BC}$ & Year 2 & Y2 & 109 & 0 & 7.77 & 0.84 & n.s. & -1.49 & -2.94 \\
\hline & Maize & $\mathrm{C}(90 \% \mathrm{~N})$ & Year 3 & Y3 & 109 & & 8.5 & & & & \\
\hline & Maize & $\mathrm{BC}$ & Year 3 & Y3 & 109 & 0 & 8.51 & 1.00 & n.s. & 0.01 & 0.20 \\
\hline & Maize & $\mathrm{BC}$ & Year 3 & Y3 & 109 & 0 & 7.93 & 0.93 & n.s. & -0.57 & -2.81 \\
\hline & Maize & $\mathrm{BC}$ & Year 3 & Y3 & 109 & 0 & 6.59 & 0.78 & n.s. & -1.91 & -3.77 \\
\hline & Maize & $\mathrm{C}(90 \% \mathrm{~N})$ & Year 4 & Y4 & 109 & & 8.69 & & & & \\
\hline & Maize & $\mathrm{BC}$ & Year 4 & Y4 & 109 & 0 & 8.6 & 0.99 & n.s. & -0.09 & -1.78 \\
\hline & Maize & $\mathrm{BC}$ & Year 4 & Y4 & 109 & 0 & 8.62 & 0.99 & n.s. & -0.07 & -0.35 \\
\hline & Maize & $\mathrm{BC}$ & Year 4 & Y4 & 109 & 0 & 7.81 & 0.90 & n.s. & -0.88 & -1.74 \\
\hline & Maize & $\mathrm{C}$ & Year $1(50 \% \mathrm{~N})$ & Y1 & 66 & & 5.66 & & & & \\
\hline & Maize & $\mathrm{BC}$ & Year 1: BC $(50 \% \mathrm{~N})$ & Y1 & 66 & 12 & 3.38 & 0.60 & n.s. & -2.28 & -11.24 \\
\hline & Maize & $\mathrm{C}$ & Year $1(70 \% \mathrm{~N})$ & Y1 & 87 & & 4.27 & & & & \\
\hline & Maize & $\mathrm{BC}$ & Year 1: BC (70\% N) & Y1 & 87 & 12 & 3.38 & 0.79 & n.s. & -0.89 & -4.39 \\
\hline & Maize & $\mathrm{C}$ & Year $1(90 \% \mathrm{~N})$ & Y1 & 109 & & 4.74 & & & & \\
\hline & Maize & $\mathrm{BC}$ & Year 1: BC (90\% N) & Y1 & 109 & 12 & 4.2 & 0.89 & n.s. & -0.54 & -2.66 \\
\hline & Maize & $\mathrm{C}$ & Year $1(100 \% \mathrm{~N})$ & Y1 & 120 & & 4.5 & & & & \\
\hline & Maize & $\mathrm{BC}$ & Year 1: BC $(100 \% \mathrm{~N})$ & Y1 & 120 & 12 & 4.66 & 1.04 & n.s. & 0.16 & 0.79 \\
\hline & Maize & $\mathrm{C}$ & Year $2(50 \% \mathrm{~N})$ & Y2 & 66 & & 6.99 & & & & \\
\hline & Maize & $\mathrm{BC}$ & Year 2: BC (50\% N) & Y2 & 66 & 0 & 6.83 & 0.98 & n.s. & -0.16 & -0.79 \\
\hline & Maize & $\mathrm{C}$ & Year $2(70 \% \mathrm{~N})$ & Y2 & 87 & & 7.37 & & & & \\
\hline & Maize & $\mathrm{BC}$ & Year 2: BC (70\% N) & Y2 & 87 & 0 & 8.28 & 1.12 & n.s. & 0.91 & 4.49 \\
\hline & Maize & $\mathrm{C}$ & Year $2(90 \% \mathrm{~N})$ & Y2 & 109 & & 9.26 & & & & \\
\hline & Maize & $\mathrm{BC}$ & Year 2: BC (90\% N) & Y2 & 109 & 0 & 8.14 & 0.88 & n.s. & -1.12 & -5.52 \\
\hline & Maize & $\mathrm{C}$ & Year $2(100 \% \mathrm{~N})$ & Y2 & 120 & & 10.41 & & & & \\
\hline & Maize & $\mathrm{BC}$ & Year 2: BC (100\% N) & Y2 & 120 & 0 & 11.38 & 1.09 & n.s. & 0.97 & 4.78 \\
\hline & Maize & $\mathrm{C}$ & Year $3(50 \% \mathrm{~N})$ & Y3 & 66 & & 6.5 & & & & \\
\hline
\end{tabular}




\begin{tabular}{|c|c|c|c|c|c|c|c|c|c|c|c|}
\hline $\begin{array}{l}\text { Ref. } \\
\text { No. }\end{array}$ & Crop & Variant $^{\mathrm{a}}$ & $\begin{array}{l}\text { Treatment, plot, site, } \\
\text { year }\end{array}$ & $\begin{array}{c}\text { Single/ } \\
\text { Multiple } \\
\text { (year) }^{b}\end{array}$ & $\begin{array}{c}\text { N ferti- } \\
\text { lizing }^{c} \\
\text { (kg N/ha) }\end{array}$ & $\begin{array}{c}\text { Biochar } \\
\text { added } \\
\text { (Mg/ha) }\end{array}$ & $\begin{array}{c}\text { Yield } \\
\text { (Mg/ha) }\end{array}$ & $\begin{array}{l}\text { Rel. } \\
\text { Yield }\end{array}$ & $\begin{array}{l}\text { Signifi- } \\
\text { cance }^{d}\end{array}$ & $\begin{array}{c}\text { Yield } \\
\text { change }^{\mathrm{e}} \\
(\mathrm{Mg} / \mathrm{ha})\end{array}$ & $\begin{array}{c}\text { Revenue }^{\mathrm{e}} \\
\text { (USD/ } \\
\text { Mg BC) }\end{array}$ \\
\hline \multirow{15}{*}{$\begin{array}{c}17 \\
\text { cont }^{6} \mathrm{~d}\end{array}$} & Maize & $\mathrm{BC}$ & Year 3: BC $(50 \% \mathrm{~N})$ & Y3 & 66 & 0 & 6.64 & 1.02 & n.s. & 0.14 & 0.69 \\
\hline & Maize & $\mathrm{C}$ & Year $3(70 \% \mathrm{~N})$ & Y3 & 87 & & 8.01 & & & & \\
\hline & Maize & $\mathrm{BC}$ & Year 3: BC (70\% N) & Y3 & 87 & 0 & 7.75 & 0.97 & n.s. & -0.26 & -1.28 \\
\hline & Maize & $\mathrm{C}$ & Year $3(90 \% \mathrm{~N})$ & Y3 & 109 & & 8.5 & & & & \\
\hline & Maize & $\mathrm{BC}$ & Year 3: BC $(90 \%$ N) & Y3 & 109 & 0 & 7.89 & 0.93 & n.s. & -0.61 & -3.01 \\
\hline & Maize & $\mathrm{C}$ & Year $3(100 \% \mathrm{~N})$ & Y3 & 120 & & 8.59 & & & & \\
\hline & Maize & $\mathrm{BC}$ & Year 3: BC (100\% N) & Y3 & 120 & 0 & 10.91 & 1.27 & n.s. & 2.32 & 11.44 \\
\hline & Maize & $\mathrm{C}$ & Year $4(50 \% \mathrm{~N})$ & Y4 & 66 & & 7.29 & & & & \\
\hline & Maize & $\mathrm{BC}$ & Year 4: BC (50\% N) & Y4 & 66 & 0 & 7.41 & 1.02 & n.s. & 0.12 & 0.59 \\
\hline & Maize & $\mathrm{C}$ & Year $4(70 \% \mathrm{~N})$ & Y4 & 87 & & 8.42 & & & & \\
\hline & Maize & $\mathrm{BC}$ & Year 4: BC (70\% N) & Y4 & 87 & 0 & 8.23 & 0.98 & n.s. & -0.19 & -0.94 \\
\hline & Maize & $\mathrm{C}$ & Year $4(90 \% \mathrm{~N})$ & Y4 & 109 & & 8.69 & & & & \\
\hline & Maize & $\mathrm{BC}$ & Year 4: BC $(90 \% \mathrm{~N})$ & Y4 & 109 & 0 & 8.62 & 0.99 & n.s. & -0.07 & -0.35 \\
\hline & Maize & $\mathrm{C}$ & Year $4(100 \% \mathrm{~N})$ & Y4 & 120 & & 8.94 & & & & \\
\hline & Maize & $\mathrm{BC}$ & Year 4: BC (100\% N) & Y4 & 120 & 0 & 8.94 & 1.00 & n.s. & 0.00 & 0.00 \\
\hline \multirow[t]{14}{*}{18} & Rice & $\mathrm{C}$ & IRRI, year 1 & Y1 & 0 & & 3.84 & & & & \\
\hline & Rice & $\mathrm{BC}$ & IRRI, year 1 & Y1 & 0 & 41.3 & 3.81 & 0.99 & n.s & -0.03 & -0.24 \\
\hline & Rice & $\mathrm{MF}$ & IRRI, year 1 & Y1 & 60 & & 5.22 & & & & \\
\hline & Rice & $\mathrm{MF}+\mathrm{BC}$ & IRRI, year 1 & Y1 & 60 & 41.3 & 4.72 & 0.90 & n.s & -0.50 & -4.00 \\
\hline & Rice & $\mathrm{C}$ & IRRI, year 2 & Y2 & 0 & & 3.02 & & & & \\
\hline & Rice & $\mathrm{BC}$ & IRRI, year 2 & Y2 & 0 & 0 & 2.89 & 0.96 & n.s & -0.13 & -1.04 \\
\hline & Rice & MF & IRRI, year 2 & Y2 & 60 & & 4.94 & & & & \\
\hline & Rice & $\mathrm{MF}+\mathrm{BC}$ & IRRI, year 2 & Y2 & 60 & 0 & 4.32 & 0.87 & $*$ & -0.62 & -4.95 \\
\hline & Rice & $\mathrm{C}$ & IRRI, year 3 & Y3 & 0 & & 3.2 & & & & \\
\hline & Rice & $\mathrm{BC}$ & IRRI, year 3 & Y3 & 0 & 0 & 3.16 & 0.99 & n.s & -0.04 & -0.32 \\
\hline & Rice & $\mathrm{MF}$ & IRRI, year 3 & Y3 & 60 & & 4.06 & & & & \\
\hline & Rice & $\mathrm{MF}+\mathrm{BC}$ & IRRI, year 3 & Y3 & 60 & 0 & 4.07 & 1.00 & n.s & 0.01 & 0.08 \\
\hline & Rice & $\mathrm{C}$ & IRRI, year 4 & Y4 & 0 & & 3.23 & & & & \\
\hline & Rice & $\mathrm{BC}$ & IRRI, year 4 & Y4 & 0 & 0 & 3.11 & 0.96 & n.s & -0.12 & -0.96 \\
\hline
\end{tabular}




\begin{tabular}{|c|c|c|c|c|c|c|c|c|c|c|c|}
\hline $\begin{array}{l}\text { Ref. } \\
\text { No. }\end{array}$ & Crop & Variant $^{\mathrm{a}}$ & $\begin{array}{l}\text { Treatment, plot, site, } \\
\text { year }\end{array}$ & $\begin{array}{c}\text { Single/ } \\
\text { Multiple } \\
\text { (year)b }^{\text {be }}\end{array}$ & $\begin{array}{c}\text { N ferti- } \\
\text { lizing } \\
(\mathrm{kg} \mathrm{N} / \mathrm{ha})\end{array}$ & $\begin{array}{c}\text { Biochar } \\
\text { added } \\
\text { (Mg/ha) }\end{array}$ & $\begin{array}{c}\text { Yield } \\
\text { (Mg/ha) }\end{array}$ & $\begin{array}{c}\text { Rel. } \\
\text { Yield }\end{array}$ & $\begin{array}{l}\text { Signifi- } \\
\text { cance }\end{array}$ & $\begin{array}{c}\text { Yield } \\
\text { change } \\
\text { (Mg/ha) }\end{array}$ & $\begin{array}{c}\text { Revenue }^{\mathrm{e}} \\
\text { (USD/ } \\
\text { Mg BC) }\end{array}$ \\
\hline \multirow{29}{*}{$\begin{array}{c}\mathbf{1 8} \\
\text { cont'd }\end{array}$} & Rice & MF & IRRI, year 4 & Y4 & 60 & & 4.24 & & & & \\
\hline & Rice & $\mathrm{MF}+\mathrm{BC}$ & IRRI, year 4 & Y4 & 60 & 0 & 3.9 & 0.92 & n.s & -0.34 & -2.72 \\
\hline & Rice & $\mathrm{C}$ & Siniloan, year 1 & Y1 & 0 & & 3.01 & & & & \\
\hline & Rice & $\mathrm{BC}$ & Siniloan, year 1 & Y1 & 0 & 41.3 & 3.52 & 1.17 & n.s & 0.51 & 4.08 \\
\hline & Rice & MF & Siniloan, year 1 & Y1 & 60 & & 2.56 & & & & \\
\hline & Rice & $\mathrm{MF}+\mathrm{BC}$ & Siniloan, year 1 & Y1 & 60 & 41.3 & 2.83 & 1.11 & n.s & 0.27 & 2.16 \\
\hline & Rice & $\mathrm{C}$ & Siniloan, year 2 & $\mathrm{Y} 2$ & 0 & & 3.01 & & & & \\
\hline & Rice & $\mathrm{BC}$ & Siniloan, year 2 & $\mathrm{Y} 2$ & 0 & 0 & 3.3 & 1.10 & n.s & 0.29 & 2.32 \\
\hline & Rice & MF & Siniloan, year 2 & $\mathrm{Y} 2$ & 60 & & 3.19 & & & & \\
\hline & Rice & $\mathrm{MF}+\mathrm{BC}$ & Siniloan, year 2 & $\mathrm{Y} 2$ & 60 & 0 & 3.29 & 1.03 & n.s & 0.10 & 0.80 \\
\hline & Rice & $\mathrm{C}$ & Siniloan, year 3 & $\mathrm{Y} 3$ & 0 & & 3.09 & & & & \\
\hline & Rice & $\mathrm{BC}$ & Siniloan, year 3 & Y3 & 0 & 0 & 3.13 & 1.01 & n.s & 0.04 & 0.32 \\
\hline & Rice & MF & Siniloan, year 3 & $\mathrm{Y} 3$ & 60 & & 3.33 & & & & \\
\hline & Rice & $\mathrm{MF}+\mathrm{BC}$ & Siniloan, year 3 & $\mathrm{Y} 3$ & 60 & 0 & 3.54 & 1.06 & n.s & 0.21 & 1.68 \\
\hline & Rice & C & Ubon, year 1 & Y1 & 0 & & 2.63 & & & & \\
\hline & Rice & $\mathrm{BC}$ & Ubon, year 1 & Y1 & 0 & 41.3 & 2.73 & 1.04 & n.s & 0.10 & 0.80 \\
\hline & Rice & MF & Ubon, year 1 & Y1 & 40 & & 3.31 & & & & \\
\hline & Rice & $\mathrm{MF}+\mathrm{BC}$ & Ubon, year 1 & Y1 & 40 & 41.3 & 3.71 & 1.12 & n.s & 0.40 & 3.20 \\
\hline & Rice & $\mathrm{C}$ & Ubon, year 2 & $\mathrm{Y} 2$ & 0 & & 2.18 & & & & \\
\hline & Rice & $\mathrm{BC}$ & Ubon, year 2 & Y2 & 0 & 0 & 2.76 & 1.27 & $*$ & 0.58 & 4.63 \\
\hline & Rice & MF & Ubon, year 2 & Y2 & 40 & & 2.63 & & & & \\
\hline & Rice & $\mathrm{MF}+\mathrm{BC}$ & Ubon, year 2 & $\mathrm{Y} 2$ & 40 & 0 & 3.33 & 1.27 & $*$ & 0.70 & 5.59 \\
\hline & Rice & $\mathrm{C}$ & Ubon, year 3 & Y3 & 0 & & 1.97 & & & & \\
\hline & Rice & $\mathrm{BC}$ & Ubon, year 3 & Y3 & 0 & 0 & 1.75 & 0.89 & n.s & -0.22 & -1.76 \\
\hline & Rice & MF & Ubon, year 3 & Y3 & 40 & & 2.00 & & & & \\
\hline & Rice & $\mathrm{MF}+\mathrm{BC}$ & Ubon, year 3 & Y3 & 40 & 0 & 2.64 & 1.32 & $*$ & 0.64 & 5.11 \\
\hline & Rice & $\mathrm{C}$ & Ubon, year 4 & Y4 & 0 & & 1.51 & & & & \\
\hline & Rice & $\mathrm{BC}$ & Ubon, year 4 & Y4 & 0 & 0 & 1.76 & 1.17 & n.s & 0.25 & 2.00 \\
\hline & Rice & MF & Ubon, year 4 & Y4 & 40 & & 2.01 & & & & \\
\hline
\end{tabular}




\begin{tabular}{|c|c|c|c|c|c|c|c|c|c|c|c|}
\hline $\begin{array}{l}\text { Ref. } \\
\text { No. }\end{array}$ & Crop & Variant $^{\mathrm{a}}$ & $\begin{array}{l}\text { Treatment, plot, site, } \\
\text { year }\end{array}$ & $\begin{array}{c}\text { Single/ } \\
\text { Multiple } \\
\text { (year) }^{b}\end{array}$ & $\begin{array}{c}\text { N ferti- } \\
\text { lizing }^{c} \\
\text { (kg N/ha) }\end{array}$ & $\begin{array}{c}\text { Biochar } \\
\text { added } \\
\text { (Mg/ha) }\end{array}$ & $\begin{array}{c}\text { Yield } \\
\text { (Mg/ha) }\end{array}$ & $\begin{array}{l}\text { Rel. } \\
\text { Yield }\end{array}$ & $\begin{array}{l}\text { Signifi- } \\
\text { cance }^{d}\end{array}$ & $\begin{array}{c}\text { Yield } \\
\text { change }^{\mathrm{e}} \\
\text { (Mg/ha) }\end{array}$ & $\begin{array}{c}\text { Revenue }^{\mathrm{e}} \\
\text { (USD/ } \\
\text { Mg BC) }\end{array}$ \\
\hline cont'd & Rice & $\mathrm{MF}+\mathrm{BC}$ & Ubon, year 4 & Y4 & 40 & 0 & 2.72 & 1.35 & n.s & 0.71 & 5.67 \\
\hline \multirow{28}{*}{$\begin{array}{l}19 \\
(\mathrm{k})\end{array}$} & Wheat & $(+\mathrm{P})$ & & Y1 & 92 & & 5.16 & & & & \\
\hline & Wheat & $\mathrm{BC}(\mathrm{WS})+\mathrm{P}$ & & Y1 & 92 & 5.0 & 5.23 & 1.01 & n.s. & 0.06 & 1.18 \\
\hline & Wheat & $\mathrm{BC}(\mathrm{CM})+\mathrm{P}$ & & Y1 & 92 & 5.0 & 5.34 & 1.03 & n.s. & 0.17 & 3.28 \\
\hline & Wheat & no $\mathrm{P}$ & & Y1 & 92 & & 4.51 & & & & \\
\hline & Wheat & $\mathrm{BC}(\mathrm{WS})$ no $\mathrm{P}$ & & Y1 & 92 & 5.0 & 5.06 & 1.12 & $*$ & 0.54 & 10.27 \\
\hline & Wheat & $\mathrm{BC}(\mathrm{CM})$ no $\mathrm{P}$ & & Y1 & 92 & 5.0 & 5.11 & 1.13 & $*$ & 0.59 & 11.23 \\
\hline & Sorghum & $(+\mathrm{P})$ & Catch crop & Y1-2 & 0 & & 1.19 & & & & \\
\hline & Sorghum & $\mathrm{BC}(\mathrm{WS})+\mathrm{P}$ & Catch crop & Y1-2 & 0 & 0 & 1.53 & 1.29 & $*$ & 0.34 & \\
\hline & Sorghum & $\mathrm{BC}(\mathrm{CM})+\mathrm{P}$ & Catch crop & Y1-2 & 0 & 0 & 1.44 & 1.21 & n.s. & 0.25 & \\
\hline & Sorghum & no $\mathrm{P}$ & Catch crop & Y1-2 & 0 & & 1.13 & & & & \\
\hline & Sorghum & $\mathrm{BC}(\mathrm{WS})$ no $\mathrm{P}$ & Catch crop & Y1-2 & 0 & 0 & 1.54 & 1.37 & $*$ & 0.41 & \\
\hline & Sorghum & $\mathrm{BC}(\mathrm{CM})$ no $\mathrm{P}$ & Catch crop & Y1-2 & 0 & 0 & 1.29 & 1.14 & n.s. & 0.16 & \\
\hline & Lupin & $(+\mathrm{P})$ & & $\mathrm{Y} 2$ & 4 & & 2.76 & & & & \\
\hline & Lupin & $\mathrm{BC}(\mathrm{WS})+\mathrm{P}$ & & Y2 & 4 & 0 & 2.70 & 0.98 & n.s. & -0.07 & -2.42 \\
\hline & Lupin & $\mathrm{BC}(\mathrm{CM})+\mathrm{P}$ & & Y2 & 4 & 0 & 3.27 & 1.18 & n.s. & 0.51 & 18.23 \\
\hline & Lupin & no $\mathrm{P}$ & & Y2 & 4 & & 2.60 & & & & \\
\hline & Lupin & $\mathrm{BC}(\mathrm{WS})$ no $\mathrm{P}$ & & Y2 & 4 & 0 & 2.31 & 0.89 & n.s. & -0.29 & -10.49 \\
\hline & Lupin & $\mathrm{BC}(\mathrm{CM})$ no $\mathrm{P}$ & & Y2 & 4 & 0 & 2.85 & 1.10 & n.s. & 0.25 & 8.93 \\
\hline & Sorghum & $(+\mathrm{P})$ & Catch crop & Y2-3 & 0 & & 3.69 & & & & \\
\hline & Sorghum & $\mathrm{BC}(\mathrm{WS})+\mathrm{P}$ & Catch crop & Y2-3 & 0 & 0 & 3.79 & 1.03 & n.s. & 0.11 & \\
\hline & Sorghum & $\mathrm{BC}(\mathrm{CM})+\mathrm{P}$ & Catch crop & Y2-3 & 0 & 0 & 4.80 & 1.30 & n.s. & 1.11 & \\
\hline & Sorghum & no $\mathrm{P}$ & Catch crop & Y2-3 & 0 & & 3.48 & & & & \\
\hline & Sorghum & $\mathrm{BC}(\mathrm{WS})$ no $\mathrm{P}$ & Catch crop & Y2-3 & 0 & 0 & 3.95 & 1.13 & n.s. & 0.47 & \\
\hline & Sorghum & $\mathrm{BC}(\mathrm{CM})$ no $\mathrm{P}$ & Catch crop & Y2-3 & 0 & 0 & 4.17 & 1.20 & n.s. & 0.69 & \\
\hline & Wheat & $(+\mathrm{P})$ & & Y3 & 92 & & 3.81 & & & & \\
\hline & Wheat & $\mathrm{BC}(\mathrm{WS})+\mathrm{P}$ & & Y3 & 92 & 0 & 3.71 & 0.98 & n.s. & -0.09 & -1.78 \\
\hline & Wheat & $\mathrm{BC}(\mathrm{CM})+\mathrm{P}$ & & Y3 & 92 & 0 & 3.81 & 1.00 & n.s. & 0.00 & -0.03 \\
\hline & Wheat & no $\mathrm{P}$ & & Y3 & 92 & & 3.52 & & & & \\
\hline
\end{tabular}




\begin{tabular}{|c|c|c|c|c|c|c|c|c|c|c|c|}
\hline $\begin{array}{l}\text { Ref. } \\
\text { No. }\end{array}$ & Crop & Variant $^{\mathrm{a}}$ & $\begin{array}{l}\text { Treatment, plot, site, } \\
\text { year }\end{array}$ & \begin{tabular}{|c|}
$\begin{array}{c}\text { Single/ } \\
\text { Multiple } \\
\text { (year) }^{b}\end{array}$ \\
\end{tabular} & $\begin{array}{c}\text { N ferti- } \\
\text { lizing }^{c} \\
\text { (kg N/ha) }\end{array}$ & $\begin{array}{c}\text { Biochar } \\
\text { added } \\
\text { (Mg/ha) }\end{array}$ & $\begin{array}{c}\text { Yield } \\
\text { (Mg/ha) }\end{array}$ & $\begin{array}{l}\text { Rel. } \\
\text { Yield }\end{array}$ & $\begin{array}{l}\text { Signifi- } \\
\text { cance }^{d}\end{array}$ & $\begin{array}{c}\text { Yield } \\
\text { change }^{\mathrm{e}} \\
(\mathrm{Mg} / \mathrm{ha})\end{array}$ & $\begin{array}{c}\text { Revenue }^{\mathrm{e}} \\
\text { (USD/ } \\
\text { Mg BC) }\end{array}$ \\
\hline $\begin{array}{c}19 \\
\text { cont'd } \\
\end{array}$ & $\begin{array}{l}\text { Wheat } \\
\text { Wheat }\end{array}$ & $\begin{array}{l}\mathrm{BC}(\mathrm{WS}) \text { no } \mathrm{P} \\
\mathrm{BC}(\mathrm{CM}) \text { no } \mathrm{P}\end{array}$ & & $\begin{array}{l}\text { Y3 } \\
\text { Y3 }\end{array}$ & $\begin{array}{l}92 \\
92 \\
\end{array}$ & $\begin{array}{l}0 \\
0 \\
\end{array}$ & $\begin{array}{l}3.52 \\
3.45 \\
\end{array}$ & $\begin{array}{l}1.00 \\
0.98 \\
\end{array}$ & $\begin{array}{l}\text { n.s. } \\
\text { n.s. }\end{array}$ & $\begin{array}{c}0.00 \\
-0.06 \\
\end{array}$ & $\begin{array}{r}0.02 \\
-1.23 \\
\end{array}$ \\
\hline \multirow{27}{*}{$\begin{array}{c}\mathbf{2 0} \\
(1) \\
(\mathrm{m})\end{array}$} & Barley & $\mathrm{C}$ & Year 1 & Y1 & n.d. & & 7.88 & & & & \\
\hline & Barley & $\mathrm{BC}$ & Year 1 & Y1 & n.d. & 10 & 8.43 & 1.07 & n.s. & 0.55 & 4.65 \\
\hline & Rapeseed & $\mathrm{C}$ & Year 2 & $\mathrm{Y} 2$ & n.d. & & 3.39 & & & & \\
\hline & Rapeseed & $\mathrm{BC}$ & Year 2 & Y2 & n.d. & 0 & 3.71 & 1.09 & n.s. & 0.32 & 16.74 \\
\hline & Wheat & $\mathrm{C}$ & Year 3 & Y3 & n.d. & & 8.81 & & & & \\
\hline & Wheat & $\mathrm{BC}$ & Year 3 & Y3 & n.d. & 0 & 9.28 & 1.05 & $*$ & 0.47 & 4.45 \\
\hline & Rapeseed & $\mathrm{C}$ & Year 1 & Y1 & n.d. & & 3.36 & & & & \\
\hline & Rapeseed & $\mathrm{BC}$ & Year 1 & Y1 & n.d. & 10 & 4.12 & 1.23 & n.s. & 0.76 & 39.76 \\
\hline & Rapeseed & $\mathrm{BC}$ & Year 1 & Y1 & n.d. & 20 & 3.72 & 1.11 & n.s. & 0.36 & 9.42 \\
\hline & Rapeseed & $\mathrm{BC}$ & Year 10 & Y1 & n.d. & 40 & 3.52 & 1.05 & n.s. & 0.16 & 2.09 \\
\hline & Wheat & $\mathrm{C}$ & Year 2 & Y2 & n.d. & & 8.78 & & & & \\
\hline & Wheat & $\mathrm{BC}$ & Year 2 & Y2 & n.d. & 0 & 9.31 & 1.06 & $*$ & 0.53 & 5.02 \\
\hline & Wheat & $\mathrm{BC}$ & Year 2 & Y2 & n.d. & 0 & 8.88 & 1.01 & n.s. & 0.10 & 0.47 \\
\hline & Wheat & $\mathrm{BC}$ & Year 2 & Y2 & n.d. & 0 & 8.51 & 0.97 & $*$ & -0.27 & -0.64 \\
\hline & Sugar beet & MF & Year 1 & $\mathrm{~S}$ & 125 & & 8.86 & & & & \\
\hline & Sugar beet & $\mathrm{MF}+\mathrm{BC}$ & Year 1 & $\mathrm{~S}$ & 125 & 10 & 8.64 & 0.98 & n.s. & -0.22 & -3.19 \\
\hline & Sugar beet & $\mathrm{MF}+\mathrm{BC}$ & Year 1 & $\mathrm{~S}$ & 125 & 20 & 10.35 & 1.17 & n.s. & 1.49 & 10.80 \\
\hline & Sugar beet & $\mathrm{MF}+\mathrm{BC}$ & Year 1 & $\mathrm{~S}$ & 125 & 40 & 8.8 & 0.99 & n.s. & -0.06 & -0.22 \\
\hline & Carrots & $\mathrm{C}$ & Year 1 & $\mathrm{~S}$ & 75 & & 16.55 & & & & \\
\hline & Carrots & $\mathrm{BC}$ & Year 1 & $\mathrm{~S}$ & 75 & 10 & 18.8 & 1.14 & n.s. & 2.25 & \\
\hline & Carrots & $\mathrm{BC}$ & Year 1 & $\mathrm{~S}$ & 75 & 20 & 17.2 & 1.04 & n.s. & 0.65 & \\
\hline & Carrots & $\mathrm{BC}$ & Year 1 & $\mathrm{~S}$ & 75 & 40 & 18.33 & 1.11 & n.s. & 1.78 & \\
\hline & Barley & $\mathrm{C}$ & & $\mathrm{S}$ & n.d. & & 8.87 & & & & \\
\hline & Barley & $\mathrm{BC}$ & BC10 & $\mathrm{S}$ & n.d. & 10 & 8.9 & 1.00 & n.s. & 0.03 & 0.25 \\
\hline & Barley & $\mathrm{BC}$ & BC20 & $\mathrm{S}$ & n.d. & 20 & 9.09 & 1.02 & n.s. & 0.22 & 0.93 \\
\hline & Barley & $\mathrm{BC}$ & BC40 & $\mathrm{S}$ & n.d. & 40 & 8.87 & 1.00 & n.s. & 0.00 & 0.00 \\
\hline & Spinach & $\mathrm{C}$ & & $\mathrm{S}$ & n.d. & & 30.61 & & & & \\
\hline
\end{tabular}




\begin{tabular}{|c|c|c|c|c|c|c|c|c|c|c|c|}
\hline $\begin{array}{l}\text { Ref. } \\
\text { No. }\end{array}$ & Crop & Variant $^{\mathbf{a}}$ & $\begin{array}{l}\text { Treatment, plot, site, } \\
\text { year }\end{array}$ & \begin{tabular}{|c|}
$\begin{array}{c}\text { Single/ } \\
\text { Multiple } \\
\text { (year) }^{b}\end{array}$ \\
\end{tabular} & $\begin{array}{c}\text { N ferti- } \\
\text { lizing }^{c} \\
\text { (kg N/ha) }\end{array}$ & $\begin{array}{c}\text { Biochar } \\
\text { added } \\
\text { (Mg/ha) }\end{array}$ & $\begin{array}{c}\text { Yield } \\
\text { (Mg/ha) }\end{array}$ & $\begin{array}{l}\text { Rel. } \\
\text { Yield }\end{array}$ & $\begin{array}{l}\text { Signifi- } \\
\text { cance }^{d}\end{array}$ & $\begin{array}{c}\text { Yield } \\
\text { change }^{\mathrm{e}} \\
(\mathrm{Mg} / \mathrm{ha})\end{array}$ & $\begin{array}{c}\text { Revenue }^{\mathrm{e}} \\
\text { (USD/ } \\
\text { Mg BC) }\end{array}$ \\
\hline \multirow{26}{*}{$\begin{array}{c}\mathbf{2 0} \\
\text { cont'd }\end{array}$} & Spinach & $\mathrm{BC}$ & BC6 & $\mathrm{S}$ & n.d. & 6 & 29.96 & 0.98 & n.s. & -0.65 & \\
\hline & Spinach & $\mathrm{BC}$ & BC12 & $S$ & n.d. & 12 & 29.23 & 0.95 & n.s. & -1.38 & \\
\hline & Spinach & $\mathrm{BC}$ & BC24 & $\mathrm{S}$ & n.d. & 24 & 29.38 & 0.96 & n.s. & -1.23 & \\
\hline & Spinach & $\mathrm{BC}$ & BC40 & $\mathrm{S}$ & n.d. & 40 & 29.34 & 0.96 & n.s. & -1.27 & \\
\hline & Spinach & $\mathrm{C}$ & & $\mathrm{S}$ & n.d. & & 31.74 & & & & \\
\hline & Spinach & $\mathrm{BC}$ & BC12: deep banded & $\mathrm{S}$ & n.d. & 12 & 26.51 & 0.87 & $*$ & -5.23 & \\
\hline & Spinach & $\mathrm{BC}$ & BC24: deep banded & $\mathrm{S}$ & n.d. & 24 & 30.71 & 1.00 & n.s. & -1.03 & \\
\hline & Spinach & $\mathrm{BC}$ & BC40: deep banded & $\mathrm{S}$ & n.d. & 40 & 28.44 & 0.93 & n.s. & -3.30 & \\
\hline & Spinach & $\mathrm{BC}$ & BC80: deep banded & $\mathrm{S}$ & n.d. & 80 & 30.86 & 1.01 & n.s. & -0.88 & \\
\hline & Wheat & $\mathrm{C}$ & & S & n.d. & & 8.316 & & & & \\
\hline & Wheat & $\mathrm{BC}$ & $\mathrm{BC}: 10$ & $\mathrm{~S}$ & n.d. & 10 & 8.696 & 1.05 & n.s. & 0.38 & 3.60 \\
\hline & Vicia faba & $\mathrm{C}$ & & $\mathrm{S}$ & n.d. & & 5.31 & & & & \\
\hline & Vicia faba & $\mathrm{BC}$ & BC: 10 & $\mathrm{~S}$ & n.d. & 10 & 7.51 & 1.41 & n.s. & 2.20 & \\
\hline & Vicia faba & $\mathrm{BC}$ & $\mathrm{BC}: 30$ & $\mathrm{~S}$ & n.d. & 30 & 5.41 & 1.02 & n.s. & 0.10 & \\
\hline & Vicia faba & $\mathrm{C}$ & & $\mathrm{S}$ & n.d. & & 5.38 & & & & \\
\hline & Vicia faba & $\mathrm{BC}$ & BC: 10 & S & n.d. & 10 & 5.28 & 1.02 & n.s. & -0.10 & \\
\hline & Vicia faba & $\mathrm{BC}$ & $\mathrm{BC}: 30$ & $\mathrm{~S}$ & n.d. & 30 & 4.65 & 1.00 & n.s. & -0.73 & \\
\hline & Pisum sativum & $\mathrm{C}$ & & $\mathrm{S}$ & n.d. & & 1.79 & & & & \\
\hline & Pisum sativum & $\mathrm{BC}$ & BC: 10 & $\mathrm{~S}$ & n.d. & 10 & 1.41 & 0.79 & n.s. & -0.38 & \\
\hline & Pisum sativum & $\mathrm{BC}$ & $\mathrm{BC}: 30$ & $\mathrm{~S}$ & n.d. & 30 & 1.35 & 0.75 & $*$ & -0.44 & \\
\hline & Pisum sativum & $\mathrm{C}$ & & S & n.d. & & 7.28 & & & & \\
\hline & Pisum sativum & $\mathrm{BC}$ & $\mathrm{BC}: 10$ & $\mathrm{~S}$ & n.d. & 10 & 7.36 & 1.01 & n.s. & 0.08 & \\
\hline & Pisum sativum & $\mathrm{BC}$ & BC: 30 & $\mathrm{~S}$ & n.d. & 30 & 8.76 & 1.20 & n.s. & 1.48 & \\
\hline & Barley & $\mathrm{C}$ & & $\mathrm{S}$ & 144 & & 4.429 & & & & \\
\hline & Barley & $\mathrm{MF}+\mathrm{BC}$ & BC: 10 & $\mathrm{~S}$ & 144 & 10 & 9.595 & 2.17 & * & 5.17 & \\
\hline & Barley & $\mathrm{MF}+\mathrm{BC}$ & $\mathrm{BC}: 30$ & $\mathrm{~S}$ & 144 & 30 & 9.111 & 2.06 & $*$ & 4.68 & \\
\hline \multirow[t]{3}{*}{21} & Maize & $\mathrm{MF}$ & Maize+Manioc - Maize & Y1 & 180 & & 3.06 & & & & \\
\hline & Maize & $\mathrm{MF}+\mathrm{BC}$ & Maize+Manioc - Maize & Y1 & 180 & 15.0 & 4.06 & 1.33 & $*$ & 1.00 & 3.94 \\
\hline & Manioc & MF & Maize+Manioc - Manioc & Y1 & 180 & & 16.44 & & & & \\
\hline
\end{tabular}




\begin{tabular}{|c|c|c|c|c|c|c|c|c|c|c|c|}
\hline $\begin{array}{l}\text { Ref. } \\
\text { No. }\end{array}$ & Crop & Variant $^{\mathrm{a}}$ & $\begin{array}{l}\text { Treatment, plot, site, } \\
\text { year }\end{array}$ & $\begin{array}{c}\text { Single/ } \\
\text { Multiple } \\
\text { (year) }^{b}\end{array}$ & $\begin{array}{c}\text { N ferti- } \\
\text { lizing }^{c} \\
\text { (kg N/ha) }\end{array}$ & $\begin{array}{c}\text { Biochar } \\
\text { added } \\
\text { (Mg/ha) }\end{array}$ & $\begin{array}{c}\text { Yield } \\
\text { (Mg/ha) }\end{array}$ & $\begin{array}{l}\text { Rel. } \\
\text { Yield }\end{array}$ & $\begin{array}{l}\text { Signifi- } \\
\text { cance }^{d}\end{array}$ & $\begin{array}{c}\text { Yield } \\
\text { change }^{\mathrm{e}} \\
(\mathrm{Mg} / \mathrm{ha})\end{array}$ & $\begin{array}{c}\text { Revenue }^{\mathrm{e}} \\
\text { (USD/ } \\
\text { Mg BC) }\end{array}$ \\
\hline \multirow{17}{*}{$\begin{array}{c}21 \\
\text { cont'd }^{6}\end{array}$} & Manihoc & $\mathrm{MF}+\mathrm{BC}$ & Maize+Manioc - Manioc & $\mathrm{Y} 1$ & 180 & 15.0 & 18.33 & 1.11 & * & 1.89 & \\
\hline & Maize & $\mathrm{MF}$ & Maize & Y1 & 180 & & 4.09 & & & & \\
\hline & Maize & $\mathrm{MF}+\mathrm{BC}$ & Maize & Y1 & 180 & 15.0 & 4.17 & 1.02 & n.s. & 0.08 & 0.32 \\
\hline & Manioc & $\mathrm{MF}$ & Manioc & Y1 & 180 & & 30.67 & & & & \\
\hline & Manihoc & $\mathrm{MF}+\mathrm{BC}$ & Manioc & Y1 & 180 & 15.0 & 32.66 & 1.06 & n.s. & 1.99 & \\
\hline & Maize & $\mathrm{MF}$ & Maize+Manioc - Maize & Y2 & 180 & & 2.73 & & & & \\
\hline & Maize & $\mathrm{MF}+\mathrm{BC}$ & Maize+Manioc - Maize & Y2 & 180 & 0 & 4.13 & 1.51 & $*$ & 1.40 & 5.52 \\
\hline & Manioc & $\mathrm{MF}$ & Maize+Manioc - Manioc & Y2 & 180 & & 14.40 & & & & \\
\hline & Manihoc & $\mathrm{MF}+\mathrm{BC}$ & Maize+Manioc - Manioc & Y2 & 180 & 0 & 23.20 & 1.61 & $*$ & 8.80 & \\
\hline & Maize & $\mathrm{MF}$ & Maize & Y2 & 180 & & 3.62 & & & & \\
\hline & Maize & $\mathrm{MF}+\mathrm{BC}$ & Maize & Y2 & 180 & 0 & 3.99 & 1.10 & $*$ & 0.37 & 1.46 \\
\hline & Manioc & $\mathrm{MF}$ & Manioc & Y2 & 180 & & 22.35 & & & & \\
\hline & Manihoc & $\mathrm{MF}+\mathrm{BC}$ & Manioc & Y2 & 180 & 0 & 32.06 & 1.43 & $*$ & 9.71 & \\
\hline & Maize & $\mathrm{MF}$ & Maize+Manioc - Maize & Y3 & 180 & & 2.06 & & & & \\
\hline & Maize & $\mathrm{MF}+\mathrm{BC}$ & Maize+Manioc - Maize & Y3 & 180 & 0 & 3.89 & 1.89 & $*$ & 1.83 & 7.22 \\
\hline & Manioc & $\mathrm{MF}$ & Maize & Y3 & 180 & & 3.06 & & & & \\
\hline & Manioc & $\mathrm{MF}+\mathrm{BC}$ & Maize & Y3 & 180 & 0 & 4.06 & 1.33 & $*$ & 1.00 & 3.94 \\
\hline \multirow{6}{*}{$\begin{array}{c}22 \\
(\mathrm{~m})\end{array}$} & Spring barley & $\mathrm{MF}$ & & $\mathrm{S}$ & 81 & & 6.69 & & & & \\
\hline & Spring barley & $\mathrm{MF}+\mathrm{BC} 20$ & & $S$ & 81 & 20.0 & 6.47 & 0.97 & n.s. & -0.22 & -0.93 \\
\hline & Spring barley & $\mathrm{MF}+\mathrm{BC} 50$ & & S & 81 & 50.0 & 5.77 & 0.86 & n.s. & -0.92 & -1.56 \\
\hline & Potatoes & $\mathrm{MF}$ & & S & 250 & & 72.0 & & & & \\
\hline & Potatoes & $\mathrm{MF}+\mathrm{BC} 20$ & & $\mathrm{~S}$ & 250 & 20.0 & 85.6 & 1.19 & n.s. & 13.60 & 123.08 \\
\hline & Potatoes & $\mathrm{MF}+\mathrm{BC} 50$ & & $\mathrm{~S}$ & 250 & 50.0 & 74.0 & 1.03 & n.s. & 2.00 & 7.24 \\
\hline \multirow[t]{6}{*}{23} & Silage maize & $\mathrm{MF}$ & Year 1; Maize & Y1 & 100 & - & 26.00 & & & & \\
\hline & Silage maize & $\mathrm{MF}+\mathrm{BC}$ & Year 1; Maisze & Y1 & 100 & 25.0 & 25.00 & 0.96 & n.s. & & \\
\hline & Silage maize & $\mathrm{MF}+\mathrm{BC}$ & Year 1; Maize & Y1 & 100 & 50.0 & 26.00 & 1.00 & n.s. & & \\
\hline & Ryegrass & $\mathrm{MF}$ & Year 2, grass & Y2 & 100 & & 4.30 & & & & \\
\hline & Ryegrass & $\mathrm{MF}+\mathrm{BC}$ & Year 2; grass & $\mathrm{Y} 2$ & 100 & 0 & 4.50 & 1.05 & n.s. & & \\
\hline & Ryegrass & $\mathrm{MF}+\mathrm{BC}$ & Year 2; grass & Y2 & 100 & 0 & 5.80 & 1.35 & n.s. & & \\
\hline
\end{tabular}




\begin{tabular}{|c|c|c|c|c|c|c|c|c|c|c|c|}
\hline $\begin{array}{l}\text { Ref. } \\
\text { No. }\end{array}$ & Crop & Variant $^{\mathrm{a}}$ & $\begin{array}{l}\text { Treatment, plot, site, } \\
\text { year }\end{array}$ & $\begin{array}{c}\text { Single/ } \\
\text { Multiple } \\
\text { (year) }^{b}\end{array}$ & $\begin{array}{c}\text { N ferti- } \\
\text { lizing } \\
(\mathrm{kg} N / h a)\end{array}$ & $\begin{array}{c}\text { Biochar } \\
\text { added } \\
\text { (Mg/ha) }\end{array}$ & $\begin{array}{c}\text { Yield } \\
\text { (Mg/ha) }\end{array}$ & $\begin{array}{l}\text { Rel. } \\
\text { Yield }\end{array}$ & $\begin{array}{l}\text { Signifi- }^{\text {cance }^{d}} \\
\end{array}$ & $\begin{array}{c}\text { Yield } \\
\text { change }^{\mathrm{e}} \\
(\mathrm{Mg} / \mathrm{ha})\end{array}$ & $\begin{array}{c}\text { Revenue }^{\mathrm{e}} \\
\text { (USD/ } \\
\text { Mg BC) }\end{array}$ \\
\hline \multirow{2}{*}{$\begin{array}{c}22 \\
\text { cont'd }\end{array}$} & Ryegrass & $\mathrm{C}$ & Year 3; grass & Y3 & 0 & & 3.80 & & & & \\
\hline & $\begin{array}{l}\text { Ryegrass } \\
\text { Ryegrass }\end{array}$ & $\begin{array}{l}\mathrm{BC} \\
\mathrm{BC}\end{array}$ & $\begin{array}{l}\text { Year 3; grass } \\
\text { Year 3; grass }\end{array}$ & $\begin{array}{l}\text { Y3 } \\
\text { Y3 }\end{array}$ & $\begin{array}{l}0 \\
0\end{array}$ & $\begin{array}{l}0 \\
0\end{array}$ & $\begin{array}{l}6.50 \\
6.80\end{array}$ & $\begin{array}{l}1.71 \\
1.79\end{array}$ & * & & \\
\hline \multirow[t]{8}{*}{24} & Barley & $\mathrm{MF}$ & Year 1 & Y1 & 120 & & 5.4 & & & & \\
\hline & $\begin{array}{l}\text { Barley } \\
\text { Barley } \\
\text { Barley }\end{array}$ & $\begin{array}{l}\mathrm{MF}+\mathrm{BC} \\
\mathrm{MF}+\mathrm{BC} \\
\mathrm{BC}\end{array}$ & $\begin{array}{l}\text { Year } 1 \\
\text { Year } 1 \\
\text { Year } 1\end{array}$ & $\begin{array}{l}\text { Y1 } \\
\text { Y1 } \\
\text { Y1 }\end{array}$ & $\begin{array}{c}120 \\
120 \\
0\end{array}$ & $\begin{array}{l}24 \\
72 \\
72\end{array}$ & $\begin{array}{l}5.2 \\
5.9 \\
4.7\end{array}$ & $\begin{array}{l}0.96 \\
1.09 \\
0.87\end{array}$ & $\begin{array}{c}\text { n.s. } \\
* \\
*\end{array}$ & $\begin{array}{l}-0.20 \\
0.50 \\
-0.70\end{array}$ & $\begin{array}{l}-0.70 \\
0.59 \\
-0.82\end{array}$ \\
\hline & Sunflower & $\mathrm{MF}$ & Year 2 & Y2 & 120 & & 3.7 & & & & \\
\hline & $\begin{array}{l}\text { Sunflower } \\
\text { Sunflower } \\
\text { Sunflower }\end{array}$ & $\begin{array}{l}\mathrm{MF}+\mathrm{BC} \\
\mathrm{MF}+\mathrm{BC} \\
\mathrm{BC}\end{array}$ & $\begin{array}{l}\text { Year } 2 \\
\text { Year } 2 \\
\text { Year } 2\end{array}$ & $\begin{array}{l}\mathrm{Y} 2 \\
\mathrm{Y} 2 \\
\mathrm{Y} 2\end{array}$ & $\begin{array}{c}120 \\
120 \\
0\end{array}$ & $\begin{array}{l}0 \\
0 \\
0\end{array}$ & $\begin{array}{c}4 \\
3.5 \\
3.4\end{array}$ & $\begin{array}{l}1.08 \\
0.95 \\
0.92\end{array}$ & $\begin{array}{l}* \\
\text { n.s. } \\
\text { n.s. }\end{array}$ & $\begin{array}{l}0.30 \\
-0.20 \\
-0.30\end{array}$ & $\begin{array}{l}5.70 \\
-1.27 \\
-1.90\end{array}$ \\
\hline & Maize & $\mathrm{C}$ & Year 1 & Y1 & 75 & & 7.1 & & & & \\
\hline & $\begin{array}{l}\text { Maize } \\
\text { Maize } \\
\text { Naize }\end{array}$ & $\begin{array}{l}\mathrm{MF}+\mathrm{BC} \\
\mathrm{MF}+\mathrm{BC} \\
\mathrm{BC}\end{array}$ & $\begin{array}{l}\text { Year } 1 \\
\text { Year } 1 \\
\text { Year } 1\end{array}$ & $\begin{array}{l}\text { Y1 } \\
\text { Y1 } \\
\text { Y1 }\end{array}$ & $\begin{array}{c}75 \\
75 \\
0\end{array}$ & $\begin{array}{l}24 \\
72 \\
72\end{array}$ & $\begin{array}{c}8.1 \\
7.8 \\
4\end{array}$ & $\begin{array}{l}1.14 \\
1.10 \\
0.56\end{array}$ & $\begin{array}{l}\text { n.s. } \\
\text { n.s. } \\
*\end{array}$ & $\begin{array}{l}1.00 \\
0.70 \\
-3.10\end{array}$ & $\begin{array}{l}2.47 \\
0.58 \\
-2.55\end{array}$ \\
\hline & Wheat & $\mathrm{C}$ & Year 2 & Y2 & 120 & & 5.3 & & & & \\
\hline & $\begin{array}{l}\text { Wheat } \\
\text { Wheat } \\
\text { Wheat }\end{array}$ & $\begin{array}{l}\mathrm{MF}+\mathrm{BC} \\
\mathrm{MF}+\mathrm{BC} \\
\mathrm{BC}\end{array}$ & $\begin{array}{l}\text { Year } 2 \\
\text { Year } 2 \\
\text { Year } 2 \\
\end{array}$ & $\begin{array}{l}\mathrm{Y} 2 \\
\mathrm{Y} 2 \\
\mathrm{Y} 2 \\
\end{array}$ & $\begin{array}{c}120 \\
120 \\
0 \\
\end{array}$ & $\begin{array}{l}0 \\
0 \\
0 \\
\end{array}$ & $\begin{array}{l}5.1 \\
5.5 \\
1.5 \\
\end{array}$ & $\begin{array}{l}0.96 \\
1.04 \\
0.28 \\
\end{array}$ & $\begin{array}{c}\text { n.s. } \\
\text { n.s. } \\
*\end{array}$ & $\begin{array}{c}-0.20 \\
0.20 \\
-3.80 \\
\end{array}$ & $\begin{array}{c}-0.79 \\
0.26 \\
-5.00 \\
\end{array}$ \\
\hline \multirow[t]{4}{*}{25} & Maize & $\mathrm{MF}$ & Clayey soil & $\mathrm{S}$ & 120 & & 6.03 & & & & \\
\hline & Maize & $\mathrm{MF}+\mathrm{BC}$ & Sandy soil & $\mathrm{S}$ & 120 & 6.0 & 6.21 & 1.03 & n.s. & 0.18 & 1.78 \\
\hline & Maize & $\mathrm{MF}$ & Clayey soil & S & 120 & & 8.53 & & & & \\
\hline & Maize & $\mathrm{MF}+\mathrm{BC}$ & Sandy soil & $\mathrm{S}$ & 120 & 6.0 & 6.64 & 0.78 & $*$ & -1.89 & -18.64 \\
\hline \multirow[t]{6}{*}{26} & Silage maize & $\mathrm{C}$ & Year 1 & Y1 & 200 & & 18.1 & & & & \\
\hline & Silage maize & $\mathrm{BC}$ & Year 1 & Y1 & 200 & 22.4 & 19.45 & 1.07 & n.s. & 1.35 & \\
\hline & Silage maize & Mic & Year 1 & Y1 & 0 & & 18 & & & & \\
\hline & Silage maize & $\mathrm{BC}+\mathrm{Mic}$ & Year 1 & Y1 & 0 & 22.4 & 18.6 & 1.03 & n.s. & 0.60 & \\
\hline & Silage maize & $\mathrm{C}$ & Year 2 & Y2 & 224 & & 23.3 & & & & \\
\hline & Silage maize & $\mathrm{BC}$ & Year 2 & Y2 & 224 & 0 & 16.1 & 0.69 & $*$ & -7.20 & \\
\hline
\end{tabular}




\begin{tabular}{|c|c|c|c|c|c|c|c|c|c|c|c|}
\hline $\begin{array}{l}\text { Ref. } \\
\text { No. }\end{array}$ & Crop & Variant $^{\mathrm{a}}$ & $\begin{array}{l}\text { Treatment, plot, site, } \\
\text { year }\end{array}$ & $\begin{array}{c}\text { Single/ } \\
\text { Multiple } \\
\text { (year) }^{b}\end{array}$ & $\begin{array}{c}\text { N ferti- } \\
\text { lizing }^{c} \\
\text { (kg N/ha) }\end{array}$ & $\begin{array}{c}\text { Biochar } \\
\text { added } \\
\text { (Mg/ha) }\end{array}$ & $\begin{array}{c}\text { Yield } \\
\text { (Mg/ha) }\end{array}$ & $\begin{array}{l}\text { Rel. } \\
\text { Yield }\end{array}$ & $\begin{array}{l}\text { Signifi- } \\
\text { cance }^{d}\end{array}$ & $\begin{array}{c}\text { Yield } \\
\text { change }^{\mathrm{e}} \\
(\mathrm{Mg} / \mathrm{ha})\end{array}$ & $\begin{array}{c}\text { Revenue }^{\mathrm{e}} \\
\text { (USD/ } \\
\text { Mg BC) }\end{array}$ \\
\hline \multirow{2}{*}{$\begin{array}{c}26 \\
\text { cont'd }\end{array}$} & Silage maize & Mic & Year 2 & Y2 & 67 & & 25.9 & & & & \\
\hline & Silage maize & $\mathrm{BC}+\mathrm{Mic}$ & Year 2 & Y2 & 67 & 0 & 26.4 & 1.13 & n.s. & 0.50 & \\
\hline \multirow[t]{18}{*}{27} & Rice & $\mathrm{C}$ & Site GH & $S$ & 0 & & 8.38 & & & & \\
\hline & Rice & BC20 & Site GH & $S$ & 0 & 20.0 & 8.18 & 0.98 & n.s. & -0.20 & -3.30 \\
\hline & Rice & BC40 & Site GH & S & 0 & 40.0 & 8.29 & 0.99 & n.s. & -0.09 & -0.74 \\
\hline & Rice & $\mathrm{MF}$ & Site GH & S & 240 & & 9.04 & & & & \\
\hline & Rice & $\mathrm{MF}+\mathrm{BC} 20$ & Site GH & $S$ & 240 & 20.0 & 9.00 & 1.00 & n.s. & -0.04 & -0.66 \\
\hline & Rice & $\mathrm{MF}+\mathrm{BC} 40$ & Site GH & S & 240 & 40.0 & 9.10 & 1.01 & n.s. & 0.06 & 0.50 \\
\hline & Rice & $\mathrm{C}$ & Site CS & S & 0 & & 6.38 & & & & \\
\hline & Rice & BC20 & Site CS & S & 0 & 20.0 & 6.36 & 1.00 & n.s. & -0.02 & -0.33 \\
\hline & Rice & BC40 & Site CS & S & 0 & 40.0 & 6.36 & 1.00 & n.s. & -0.02 & -0.17 \\
\hline & Rice & $\mathrm{MF}$ & Site CS & S & 240 & & 7.85 & & & & \\
\hline & Rice & $\mathrm{MF}+\mathrm{BC} 20$ & Site CS & S & 240 & 20.0 & 7.74 & 0.99 & n.s. & -0.11 & -1.82 \\
\hline & Rice & $\mathrm{MF}+\mathrm{BC} 40$ & Site CS & S & 240 & 40.0 & 8.17 & 1.04 & n.s. & 0.32 & 2.64 \\
\hline & Rice & $\mathrm{C}$ & Site JX & S & 0 & & 6.67 & & & & \\
\hline & Rice & BC20 & Site JX & $\mathrm{S}$ & 0 & 20.0 & 6.63 & 0.99 & n.s. & -0.04 & -0.66 \\
\hline & Rice & BC40 & Site JX & S & 0 & 40.0 & 6.80 & 1.02 & n.s. & 0.13 & 1.07 \\
\hline & Rice & $\mathrm{MF}$ & Site JX & S & 300 & & 7.92 & & & & \\
\hline & Rice & $\mathrm{MF}+\mathrm{BC} 20$ & Site JX & $S$ & 300 & 20.0 & 8.33 & 1.05 & $*$ & 0.41 & 6.77 \\
\hline & Rice & $\mathrm{MF}+\mathrm{BC} 40$ & Site JX & S & 300 & 40.0 & 8.55 & 1.08 & $*$ & 0.63 & 5.20 \\
\hline \multirow{9}{*}{$\begin{array}{l}\mathbf{2 8} \\
(\mathrm{n})\end{array}$} & Maize & $\mathrm{MF}$ & & Y1 & 300 & & 6.65 & & & & \\
\hline & Maize & $\mathrm{MF}+\mathrm{BC} 20$ & & Y1 & 300 & 20.0 & 7.86 & 1.18 & $*$ & 1.21 & 3.58 \\
\hline & Maize & $\mathrm{MF}+\mathrm{BC} 40$ & & Y1 & 300 & 40.0 & 7.42 & 1.12 & $*$ & 0.77 & 1.14 \\
\hline & Wheat & $\mathrm{MF}$ & & Y2 & 300 & & 7.62 & & & & \\
\hline & Wheat & $\mathrm{MF}+\mathrm{BC} 20$ & & Y2 & 300 & 0 & 7.45 & 0.98 & n.s. & -0.17 & -0.81 \\
\hline & Wheat & $\mathrm{MF}+\mathrm{BC} 40$ & & Y2 & 300 & 0 & 7.87 & 1.03 & n.s. & 0.25 & 0.59 \\
\hline & Wheat & MF & & Y4 & 300 & & 7.81 & & & & \\
\hline & Wheat & $\mathrm{MF}+\mathrm{BC} 20$ & & Y4 & 300 & 0 & 7.83 & 1.00 & n.s. & 0.02 & 0.09 \\
\hline & Wheat & $\mathrm{MF}+\mathrm{BC} 40$ & & Y4 & 300 & 0 & 8.07 & 1.03 & n.s. & 0.26 & 0.62 \\
\hline
\end{tabular}




\begin{tabular}{|c|c|c|c|c|c|c|c|c|c|c|c|}
\hline $\begin{array}{l}\text { Ref. } \\
\text { No. }\end{array}$ & Crop & Variant $^{\mathrm{a}}$ & $\begin{array}{l}\text { Treatment, plot, site, } \\
\text { year }\end{array}$ & $\begin{array}{c}\text { Single/ } \\
\text { Multiple } \\
\text { (year) }^{b}\end{array}$ & $\begin{array}{c}\text { N ferti- } \\
\text { lizing } \\
(\mathrm{kg} N / h a)\end{array}$ & $\begin{array}{c}\text { Biochar } \\
\text { added } \\
\text { (Mg/ha) }\end{array}$ & $\begin{array}{c}\text { Yield } \\
\text { (Mg/ha) }\end{array}$ & $\begin{array}{l}\text { Rel. } \\
\text { Yield }\end{array}$ & $\begin{array}{l}\text { Signifi- }^{\text {cance }^{d}} \\
\end{array}$ & $\begin{array}{c}\text { Yield } \\
\text { change }^{\mathrm{e}} \\
(\mathrm{Mg} / \mathrm{ha})\end{array}$ & $\begin{array}{c}\text { Revenue }^{\mathrm{e}} \\
\text { (USD/ } \\
\text { Mg BC) }\end{array}$ \\
\hline \multirow{2}{*}{$\begin{array}{c}28 \\
\text { cont'd }\end{array}$} & Maize & $\mathrm{MF}$ & & Y5 & 300 & & 8.31 & & & & \\
\hline & $\begin{array}{l}\text { Maize } \\
\text { Maize }\end{array}$ & $\begin{array}{l}\mathrm{MF}+\mathrm{BC} 20 \\
\mathrm{MF}+\mathrm{BC} 40\end{array}$ & & $\begin{array}{l}\text { Y5 } \\
\text { Y5 }\end{array}$ & $\begin{array}{l}300 \\
300\end{array}$ & $\begin{array}{l}0 \\
0\end{array}$ & $\begin{array}{l}8.35 \\
9.11\end{array}$ & $\begin{array}{l}1.00 \\
1.10\end{array}$ & $\begin{array}{l}\text { n.s. } \\
\text { n.s. }\end{array}$ & $\begin{array}{l}0.04 \\
0.80\end{array}$ & $\begin{array}{l}0.12 \\
1.18\end{array}$ \\
\hline \multirow[t]{4}{*}{29} & Rapeseed & $\mathrm{MF}$ & & Y1 & 90 & & 1.38 & & & & \\
\hline & $\begin{array}{l}\text { Rapeseed } \\
\text { Rapeseed } \\
\text { Rapeseed } \\
\text { Rapeseed } \\
\text { Rapeseed } \\
\text { Rapeseed }\end{array}$ & $\begin{array}{l}\mathrm{MF}+\mathrm{BC} 2.5 \\
\mathrm{MF}+\mathrm{BC} 5 \\
\mathrm{MF}+\mathrm{BC} 10 \\
\mathrm{MF}+\mathrm{BC} 20 \\
\mathrm{MF}+\mathrm{BC} 30 \\
\mathrm{MF}+\mathrm{BC} 40\end{array}$ & & $\begin{array}{l}\text { Y1 } \\
\text { Y1 } \\
\text { Y1 } \\
\text { Y1 } \\
\text { Y1 } \\
\text { Y1 }\end{array}$ & $\begin{array}{l}90 \\
90 \\
90 \\
90 \\
90 \\
90\end{array}$ & $\begin{array}{c}2.5 \\
5 \\
10 \\
20 \\
30 \\
40\end{array}$ & $\begin{array}{l}1.67 \\
1.68 \\
1.91 \\
1.85 \\
2.07 \\
2.19\end{array}$ & $\begin{array}{l}1.21 \\
1.22 \\
1.38 \\
1.34 \\
1.50 \\
1.59\end{array}$ & $\begin{array}{l}* \\
* \\
* \\
* \\
* \\
*\end{array}$ & $\begin{array}{l}0.29 \\
0.30 \\
0.53 \\
0.47 \\
0.69 \\
0.81\end{array}$ & $\begin{array}{l}60.67 \\
31.38 \\
27.72 \\
12.29 \\
12.03 \\
10.59\end{array}$ \\
\hline & sweet potato & $\mathrm{MF}$ & & Y2 & 90 & & 2.07 & & & & \\
\hline & $\begin{array}{l}\text { sweet potato } \\
\text { sweet potato } \\
\text { sweet potato } \\
\text { sweet potato } \\
\text { sweet potato } \\
\text { sweet potato }\end{array}$ & $\begin{array}{l}\mathrm{MF}+\mathrm{BC} 2.5 \\
\mathrm{MF}+\mathrm{BC} 5 \\
\mathrm{MF}+\mathrm{BC} 10 \\
\mathrm{MF}+\mathrm{BC} 20 \\
\mathrm{MF}+\mathrm{BC} 30 \\
\mathrm{MF}+\mathrm{BC} 40\end{array}$ & & $\begin{array}{l}\mathrm{Y} 2 \\
\mathrm{Y} 2 \\
\mathrm{Y} 2 \\
\mathrm{Y} 2 \\
\mathrm{Y} 2 \\
\mathrm{Y} 2\end{array}$ & $\begin{array}{l}90 \\
90 \\
90 \\
90 \\
90 \\
90 \\
\end{array}$ & $\begin{array}{l}0 \\
0 \\
0 \\
0 \\
0 \\
0\end{array}$ & $\begin{array}{l}2.00 \\
2.29 \\
3.53 \\
3.78 \\
4.15 \\
4.47 \\
\end{array}$ & $\begin{array}{l}0.97 \\
1.11 \\
1.71 \\
1.83 \\
2.00 \\
2.16 \\
\end{array}$ & $\begin{array}{l}* \\
* \\
* \\
* \\
* \\
*\end{array}$ & $\begin{array}{l}-0.07 \\
0.22 \\
1.46 \\
1.71 \\
2.08 \\
2.40 \\
\end{array}$ & \\
\hline \multirow{8}{*}{$\begin{array}{l}30 \\
\text { (o) }\end{array}$} & Maize & $\mathrm{MF}$ & Year 1 & Y1 & 165 & & 4.94 & & & & \\
\hline & $\begin{array}{l}\text { Maize } \\
\text { Maize }\end{array}$ & $\begin{array}{l}\mathrm{MF}+\mathrm{BC} \\
\mathrm{MF}+\mathrm{BC}\end{array}$ & $\begin{array}{l}\text { Year } 1 \\
\text { Year } 1\end{array}$ & $\begin{array}{l}\text { Y1 } \\
\text { Y1 }\end{array}$ & $\begin{array}{l}165 \\
165\end{array}$ & $\begin{array}{c}8.0 \\
20.0\end{array}$ & $\begin{array}{l}4.88 \\
4.82\end{array}$ & $\begin{array}{l}0.99 \\
0.98\end{array}$ & $\begin{array}{l}\text { n.s. } \\
\text { n.s. }\end{array}$ & $\begin{array}{l}-0.06 \\
-0.12\end{array}$ & $\begin{array}{l}-0.44 \\
-0.36\end{array}$ \\
\hline & Maize & MF & Year 2 & Y2 & 170 & & 5.00 & & & & \\
\hline & $\begin{array}{l}\text { Maize } \\
\text { Maize }\end{array}$ & $\begin{array}{l}\mathrm{MF}+\mathrm{BC} \\
\mathrm{MF}+\mathrm{BC}\end{array}$ & $\begin{array}{l}\text { Year } 2 \\
\text { Year } 2\end{array}$ & $\begin{array}{l}\mathrm{Y} 2 \\
\mathrm{Y} 2\end{array}$ & $\begin{array}{l}170 \\
170\end{array}$ & $\begin{array}{l}0 \\
0\end{array}$ & $\begin{array}{l}5.94 \\
6.44\end{array}$ & $\begin{array}{l}1.19 \\
1.29\end{array}$ & * & $\begin{array}{l}0.94 \\
1.44\end{array}$ & $\begin{array}{l}6.95 \\
4.26\end{array}$ \\
\hline & Maize & $\mathrm{MF}$ & Year 3 & Y3 & 156 & & 5.76 & & & & \\
\hline & $\begin{array}{l}\text { Maize } \\
\text { Maize }\end{array}$ & $\begin{array}{l}\mathrm{MF}+\mathrm{BC} \\
\mathrm{MF}+\mathrm{BC}\end{array}$ & $\begin{array}{l}\text { Year } 3 \\
\text { Year } 3\end{array}$ & $\begin{array}{l}\text { Y3 } \\
\text { Y3 }\end{array}$ & $\begin{array}{l}156 \\
156\end{array}$ & $\begin{array}{l}0 \\
0\end{array}$ & $\begin{array}{l}6.59 \\
7.50\end{array}$ & $\begin{array}{l}1.14 \\
1.30\end{array}$ & $\begin{array}{c}\text { n.s. } \\
*\end{array}$ & $\begin{array}{l}0.83 \\
1.74\end{array}$ & $\begin{array}{l}6.14 \\
5.15\end{array}$ \\
\hline & Maize & $\mathrm{MF}$ & Year 4 & Y4 & 159 & & 1.85 & & & & \\
\hline & $\begin{array}{l}\text { Maize } \\
\text { Maize }\end{array}$ & $\begin{array}{l}\mathrm{MF}+\mathrm{BC} \\
\mathrm{MF}+\mathrm{BC}\end{array}$ & $\begin{array}{l}\text { Year } 4 \\
\text { Year } 4\end{array}$ & $\begin{array}{l}\text { Y4 } \\
\text { Y4 }\end{array}$ & $\begin{array}{l}159 \\
159\end{array}$ & $\begin{array}{l}0 \\
0\end{array}$ & $\begin{array}{l}3.12 \\
4.41\end{array}$ & $\begin{array}{l}1.69 \\
2.38\end{array}$ & * & $\begin{array}{l}1.27 \\
2.56\end{array}$ & $\begin{array}{l}9.39 \\
7.57\end{array}$ \\
\hline
\end{tabular}




\begin{tabular}{|c|c|c|c|c|c|c|c|c|c|c|c|}
\hline $\begin{array}{l}\text { Ref. } \\
\text { No. }\end{array}$ & Crop & Variant $^{\mathrm{a}}$ & $\begin{array}{l}\text { Treatment, plot, site, } \\
\text { year }\end{array}$ & $\begin{array}{c}\text { Single/ } \\
\text { Multiple } \\
\text { (year) }^{b}\end{array}$ & $\begin{array}{c}\text { N ferti- } \\
\text { lizing }^{c} \\
\text { (kg N/ha) }\end{array}$ & $\begin{array}{c}\text { Biochar } \\
\text { added } \\
\text { (Mg/ha) }\end{array}$ & $\begin{array}{c}\text { Yield } \\
\text { (Mg/ha) }\end{array}$ & $\begin{array}{l}\text { Rel. } \\
\text { Yield }\end{array}$ & $\begin{array}{l}\text { Signifi- } \\
\text { cance }^{d}\end{array}$ & $\begin{array}{c}\text { Yield } \\
\text { change }^{\mathrm{e}} \\
\text { (Mg/ha) }\end{array}$ & $\begin{array}{c}\text { Revenue }^{\mathrm{e}} \\
\text { (USD/ } \\
\text { Mg BC) }\end{array}$ \\
\hline \multirow[t]{29}{*}{31} & Maize & $\mathrm{Co}(\mathrm{C})$ & Forchheim (2011) & Y1 & 140 & & 12.30 & & & & \\
\hline & Maize & $\mathrm{Co}+\mathrm{HTC}$ & Forchheim (2011) & Y1 & 140 & 20 & 11.12 & 0.90 & n.s. & -1.19 & -3.52 \\
\hline & Maize & $\mathrm{Co}+$ Pyro & Forchheim (2011) & Y1 & 140 & 20 & 12.04 & 0.98 & n.s. & -0.27 & -0.79 \\
\hline & Maize & $\operatorname{Dig}(\mathrm{C})$ & Forchheim (2011) & Y1 & 110 & & 11.89 & & & & \\
\hline & Maize & Dig+HTC & Forchheim (2011) & Y1 & 110 & 20 & 10.64 & 0.90 & n.s. & -1.24 & -3.67 \\
\hline & Maize & Dig+Pyro & Forchheim (2011) & Y1 & 110 & 20 & 11.82 & 0.99 & n.s. & -0.06 & -0.19 \\
\hline & Maize & $\mathrm{MF}(\mathrm{C})$ & Forchheim (2011) & Y1 & 160 & & 11.52 & & & & \\
\hline & Maize & $\mathrm{MF}+\mathrm{HTC}$ & Forchheim (2011) & Y1 & 160 & 20 & 12.41 & 1.08 & n.s. & 0.88 & 2.61 \\
\hline & Maize & MF+Pyro & Forchheim (2011) & Y1 & 160 & 20 & 12.03 & 1.04 & n.s. & 0.50 & 1.49 \\
\hline & Wheat & $\mathrm{Co}(\mathrm{C})$ & Forchheim (2012) & Y2 & 150 & & 8.38 & & & & \\
\hline & Wheat & $\mathrm{Co}+\mathrm{HTC}$ & Forchheim (2012) & Y2 & 150 & 0 & 8.18 & 0.98 & n.s. & -0.20 & -0.95 \\
\hline & Wheat & Co+Pyro & Forchheim (2012) & Y2 & 150 & 0 & 8.61 & 1.03 & n.s. & 0.23 & 1.08 \\
\hline & Wheat & $\operatorname{Dig}(\mathrm{C})$ & Forchheim (2012) & Y2 & 150 & & 7.18 & & & & \\
\hline & Wheat & Dig+HTC & Forchheim (2012) & Y2 & 150 & 0 & 8.07 & 1.12 & n.s. & 0.89 & 4.20 \\
\hline & Wheat & Dig+Pyro & Forchheim (2012) & Y2 & 150 & 0 & 7.99 & 1.11 & n.s. & 0.81 & 3.86 \\
\hline & Wheat & $\mathrm{MF}(\mathrm{C})$ & Forchheim (2012) & Y2 & 150 & & 8.23 & & & & \\
\hline & Wheat & $\mathrm{MF}+\mathrm{HTC}$ & Forchheim (2012) & Y2 & 150 & 0 & 8.39 & 1.02 & n.s. & 0.16 & 0.75 \\
\hline & Wheat & MF+Pyro & Forchheim (2012) & Y2 & 150 & 0 & 7.68 & 0.93 & n.s. & -0.56 & -2.64 \\
\hline & Barley & $\operatorname{Co}(\mathrm{C})$ & Forchheim (2013) & Y3 & 105 & & 6.34 & & & & \\
\hline & Barley & $\mathrm{Co}+\mathrm{HTC}$ & Forchheim (2013) & Y3 & 105 & 0 & 5.98 & 0.94 & n.s. & -0.36 & -1.51 \\
\hline & Barley & Co+Pyro & Forchheim (2013) & Y3 & 105 & 0 & 6.31 & 1.00 & n.s. & -0.03 & -0.12 \\
\hline & Barley & $\operatorname{Dig}(\mathrm{C})$ & Forchheim (2013) & Y3 & 105 & & 5.94 & & & & \\
\hline & Barley & Dig+HTC & Forchheim (2013) & Y3 & 105 & 0 & 5.84 & 0.98 & n.s. & -0.10 & -0.42 \\
\hline & Barley & Dig+Pyro & Forchheim (2013) & Y3 & 105 & 0 & 4.76 & 0.80 & n.s. & -1.18 & -4.97 \\
\hline & Barley & $\mathrm{MF}(\mathrm{C})$ & Forchheim (2013) & Y3 & 105 & & 4.86 & & & & \\
\hline & Barley & $\mathrm{MF}+\mathrm{HTC}$ & Forchheim (2013) & Y3 & 105 & 0 & 5.45 & 1.12 & n.s. & 0.59 & 2.49 \\
\hline & Barley & MF+Pyro & Forchheim (2013) & Y3 & 105 & 0 & 5.11 & 1.05 & n.s. & 0.25 & 1.06 \\
\hline & Maize & $\mathrm{Co}(\mathrm{C})$ & March (2011) & Y1 & 140 & & 17.42 & & & & \\
\hline & Maize & $\mathrm{Co}+\mathrm{HTC}$ & March (2011) & Y1 & 140 & 20 & 17.71 & 1.02 & n.s. & 0.29 & 0.86 \\
\hline
\end{tabular}




\begin{tabular}{|c|c|c|c|c|c|c|c|c|c|c|c|}
\hline $\begin{array}{l}\text { Ref. } \\
\text { No. }\end{array}$ & Crop & Variant $^{\mathrm{a}}$ & $\begin{array}{l}\text { Treatment, plot, site, } \\
\text { year }\end{array}$ & $\begin{array}{c}\text { Single/ } \\
\text { Multiple } \\
\text { (year) }^{b}\end{array}$ & $\begin{array}{c}\text { N ferti- } \\
\text { lizing }^{c} \\
\text { (kg N/ha) }\end{array}$ & $\begin{array}{c}\text { Biochar } \\
\text { added } \\
\text { (Mg/ha) }\end{array}$ & $\begin{array}{c}\text { Yield } \\
\text { (Mg/ha) }\end{array}$ & $\begin{array}{l}\text { Rel. } \\
\text { Yield }\end{array}$ & $\begin{array}{l}\text { Signifi- } \\
\text { cance }^{d}\end{array}$ & $\begin{array}{c}\text { Yield } \\
\text { change }^{\mathrm{e}} \\
\text { (Mg/ha) }\end{array}$ & $\begin{array}{c}\text { Revenue }^{\mathrm{e}} \\
\text { (USD/ } \\
\text { Mg BC) }\end{array}$ \\
\hline \multirow{25}{*}{$\begin{array}{c}31 \\
\text { cont'd }\end{array}$} & Maize & Co+Pyro & March (2011) & Y1 & 140 & 20 & 19.14 & 1.10 & n.s. & 1.72 & 5.10 \\
\hline & Maize & $\operatorname{Dig}(\mathrm{C})$ & March (2011) & Y1 & 110 & & 17.84 & & & & \\
\hline & Maize & Dig+HTC & March (2011) & Y1 & 110 & 20 & 17.99 & 1.01 & n.s. & 0.16 & 0.46 \\
\hline & Maize & Dig+Pyro & March (2011) & Y1 & 110 & 20 & 17.39 & 0.97 & n.s. & -0.45 & -1.33 \\
\hline & Maize & $\mathrm{MF}(\mathrm{C})$ & March (2011) & Y1 & 160 & & 17.32 & & & & \\
\hline & Maize & $\mathrm{MF}+\mathrm{HTC}$ & March (2011) & Y1 & 160 & 20 & 18.39 & 1.06 & n.s. & 1.07 & 3.15 \\
\hline & Maize & MF+Pyro & March (2011) & Y1 & 160 & 20 & 17.64 & 1.02 & n.s. & 0.32 & 0.94 \\
\hline & Maize & $\mathrm{Co}(\mathrm{C})$ & March (2012) & Y2 & 160 & & 16.34 & & & & \\
\hline & Maize & $\mathrm{Co}+\mathrm{HTC}$ & March (2012) & Y2 & 160 & 0 & 16.69 & 1.02 & n.s. & 0.35 & 1.02 \\
\hline & Maize & Co+Pyro & March (2012) & Y2 & 160 & 0 & 18.30 & 1.12 & n.s. & 1.95 & 5.78 \\
\hline & Maize & $\operatorname{Dig}(\mathrm{C})$ & March (2012) & Y2 & 160 & & 15.97 & & & & \\
\hline & Maize & Dig+HTC & March (2012) & Y2 & 160 & 0 & 16.81 & 1.05 & n.s. & 0.84 & 2.49 \\
\hline & Maize & Dig+Pyro & March (2012) & Y2 & 160 & 0 & 16.07 & 1.01 & n.s. & 0.09 & 0.28 \\
\hline & Maize & $\mathrm{MF}(\mathrm{C})$ & March (2012) & Y2 & 160 & & 16.74 & & & & \\
\hline & Maize & $\mathrm{MF}+\mathrm{HTC}$ & March (2012) & Y2 & 160 & 0 & 15.99 & 0.96 & n.s. & -0.75 & -2.21 \\
\hline & Maize & MF+Pyro & March (2012) & Y2 & 160 & 0 & 16.79 & 1.00 & n.s. & 0.06 & 0.16 \\
\hline & Maize & $\operatorname{Co}(\mathrm{C})$ & March (2013) & Y3 & 130 & & 12.11 & & & & \\
\hline & Maize & $\mathrm{Co}+\mathrm{HTC}$ & March (2013) & Y3 & 130 & 0 & 11.27 & 0.93 & n.s. & -0.84 & -2.49 \\
\hline & Maize & Co+Pyro & March (2013) & Y3 & 130 & 0 & 10.76 & 0.89 & n.s. & -1.35 & -4.00 \\
\hline & Maize & $\operatorname{Dig}(\mathrm{C})$ & March (2013) & Y3 & 130 & & 12.00 & & & & \\
\hline & Maize & Dig+HTC & March (2013) & Y3 & 130 & 0 & 11.62 & 0.97 & n.s. & -0.38 & -1.13 \\
\hline & Maize & Dig+Pyro & March (2013) & Y3 & 130 & 0 & 12.06 & 1.00 & n.s. & 0.05 & 0.15 \\
\hline & Maize & $\mathrm{MF}(\mathrm{C})$ & March (2013) & Y3 & 130 & & 12.58 & & & & \\
\hline & Maize & $\mathrm{MF}+\mathrm{HTC}$ & March (2013) & Y3 & 130 & 0 & 12.27 & 0.98 & n.s. & -0.31 & -0.92 \\
\hline & Maize & $\mathrm{MF}+\mathrm{Pyro}$ & March (2013) & Y3 & 130 & 0 & 12.60 & 1.00 & n.s. & 0.02 & 0.05 \\
\hline \multirow[t]{4}{*}{32} & Rice & $\mathrm{C}$ & & $\mathrm{S}$ & 0 & & 0.02 & & & & \\
\hline & Rice & $\mathrm{BC}$ & & $\mathrm{S}$ & 0 & 8.3 & 0.10 & 6.67 & $*$ & 0.09 & 3.38 \\
\hline & Rice & $\mathrm{MF}+\mathrm{Ca}$ & & $\mathrm{S}$ & 30 & & 1.36 & & & & \\
\hline & Rice & $\mathrm{MF}+\mathrm{BC}+\mathrm{Ca}$ & & $\mathrm{S}$ & 30 & 8.3 & 1.79 & 1.31 & n.s. & 0.43 & 17.02 \\
\hline
\end{tabular}




\begin{tabular}{|c|c|c|c|c|c|c|c|c|c|c|c|}
\hline $\begin{array}{l}\text { Ref. } \\
\text { No. }\end{array}$ & Crop & Variant $^{\mathrm{a}}$ & $\begin{array}{l}\text { Treatment, plot, site, } \\
\text { year }\end{array}$ & $\begin{array}{c}\text { Single/ } \\
\text { Multiple } \\
\text { (year) }^{b}\end{array}$ & $\begin{array}{c}\text { N ferti- } \\
\text { lizing } \\
(\mathrm{kg} N / h a)\end{array}$ & $\begin{array}{c}\text { Biochar } \\
\text { added } \\
\text { (Mg/ha) }\end{array}$ & $\begin{array}{c}\text { Yield } \\
\text { (Mg/ha) }\end{array}$ & $\begin{array}{l}\text { Rel. } \\
\text { Yield }\end{array}$ & $\begin{array}{l}\text { Signifi- } \\
\text { cance }^{d}\end{array}$ & $\begin{array}{c}\text { Yield } \\
\text { change }^{\mathrm{e}} \\
(\mathrm{Mg} / \mathrm{ha})\end{array}$ & $\begin{array}{c}\text { Revenue }^{\mathrm{e}} \\
\text { (USD/ } \\
\text { Mg BC) }\end{array}$ \\
\hline \multirow{6}{*}{$\begin{array}{c}32 \\
\text { cont'd }\end{array}$} & Rice & $\mathrm{Co}$ & & $\mathrm{S}$ & 0 & & 5.33 & & & & \\
\hline & Rice & $\mathrm{BC}+\mathrm{Co}$ & & S & 0 & 4.2 & 2.87 & 0.54 & $*$ & -2.45 & -192.83 \\
\hline & Rice & $\mathrm{BC}+\mathrm{Co}$ & & S & 0 & 8.3 & 3.72 & 0.70 & n.s. & -1.61 & -63.94 \\
\hline & Rice & $\mathrm{Co}+\mathrm{MF}+\mathrm{Ca}$ & & S & 30 & & 7.26 & & & & \\
\hline & Rice & $\mathrm{MF}+\mathrm{BC}+\mathrm{Co}+\mathrm{Ca}$ & & S & 30 & 4.2 & 5.21 & 0.72 & n.s. & -2.05 & -161.32 \\
\hline & Rice & $\mathrm{MF}+\mathrm{BC}+\mathrm{Co}+\mathrm{Ca}$ & & $\mathrm{S}$ & 30 & 8.3 & 4.18 & 0.58 & n.s. & -3.08 & -122.63 \\
\hline \multirow[t]{4}{*}{33} & Spring barley & $\mathrm{MF}$ & 2012 & Y1 & 70 & & 5.90 & & & & \\
\hline & Spring barley & $\mathrm{MF}+\mathrm{BC} 20$ & 2012 & Y1 & 70 & 20.0 & 5.87 & 0.99 & n.s. & -0.03 & -0.13 \\
\hline & Rye & $\mathrm{MF}$ & 2013 & Y2 & 70 & & 4.87 & & & & \\
\hline & Rye & $\mathrm{MF}+\mathrm{BC} 20$ & 2013 & Y2 & 70 & 0 & 4.48 & 0.92 & n.s. & -0.39 & -6.44 \\
\hline \multirow[t]{12}{*}{34} & Maize & $\mathrm{MF}$ & 2011 & Y1 & 377 & & 6.92 & & & & \\
\hline & Maize & $\mathrm{MF}+\mathrm{BC} 19$ & 2011 & Y1 & 377 & 19.2 & 7.66 & 1.11 & n.s. & 0.74 & 2.28 \\
\hline & Maize & $\mathrm{MF}+\mathrm{BC} 38$ & 2011 & Y1 & 377 & 38.3 & 8.11 & 1.17 & n.s. & 1.19 & 1.84 \\
\hline & Maize & $\mathrm{MF}+\mathrm{BC} 57$ & 2011 & Y1 & 377 & 57.5 & 10.30 & 1.49 & $*$ & 3.38 & 3.48 \\
\hline & Maize & $\mathrm{MF}+\mathrm{BC} 77$ & 2011 & Y1 & 377 & 76.6 & 10.15 & 1.47 & $*$ & 3.23 & 2.50 \\
\hline & Maize & $\mathrm{MF}+\mathrm{BC} 96$ & 2011 & Y1 & 377 & 95.8 & 10.68 & 1.54 & $*$ & 3.76 & 2.32 \\
\hline & Maize & $\mathrm{MF}$ & 2012 & Y2 & 264 & & 7.65 & & & & \\
\hline & Maize & $\mathrm{MF}+\mathrm{BC} 19$ & 2012 & Y2 & 264 & 0 & 9.09 & 1.19 & n.s. & 1.44 & 4.44 \\
\hline & Maize & $\mathrm{MF}+\mathrm{BC} 38$ & 2012 & Y2 & 264 & 0 & 10.39 & 1.36 & n.s. & 2.74 & 4.23 \\
\hline & Maize & $\mathrm{MF}+\mathrm{BC} 57$ & 2012 & Y2 & 264 & 0 & 7.82 & 1.02 & n.s. & 0.17 & 0.17 \\
\hline & Maize & $\mathrm{MF}+\mathrm{BC} 77$ & 2012 & Y2 & 264 & 0 & 7.81 & 1.02 & n.s. & 0.16 & 0.12 \\
\hline & Maize & $\mathrm{MF}+\mathrm{BC} 96$ & 2012 & Y2 & 264 & 0 & 7.18 & 0.94 & n.s. & -0.47 & -0.29 \\
\hline \multirow[t]{7}{*}{35} & Ryegrass & $\mathrm{C}$ & & Y1 & 0 & & 3.68 & & & & \\
\hline & Ryegrass & $\mathrm{BC}$ & $\mathrm{BC}($ manure $)$ & Y1 & 0 & 10.0 & 4.58 & 1.25 & n.s. & 0.91 & \\
\hline & Ryegrass & $\mathrm{BC}$ & BC(slurry) & Y1 & 0 & 10.0 & 3.68 & 1.00 & n.s. & 0.01 & \\
\hline & Ryegrass & $\mathrm{MF}$ & & Y1 & 368 & & 7.44 & & & & \\
\hline & Ryegrass & $\mathrm{MF}+\mathrm{BC}$ & $\mathrm{BC}($ manure $)$ & Y1 & 368 & 10.0 & 8.59 & 1.15 & n.s. & 1.15 & \\
\hline & Ryegrass & $\mathrm{MF}+\mathrm{BC}$ & BC(slurry) & Y1 & 368 & 10.0 & 7.34 & 0.99 & n.s. & -0.11 & \\
\hline & Ryegrass & $\mathrm{Ca}$ & & Y2 & 0 & & 3.65 & & & & \\
\hline
\end{tabular}




\begin{tabular}{|c|c|c|c|c|c|c|c|c|c|c|c|}
\hline $\begin{array}{l}\text { Ref. } \\
\text { No. }\end{array}$ & Crop & Variant $^{\mathrm{a}}$ & $\begin{array}{l}\text { Treatment, plot, site, } \\
\text { year }\end{array}$ & $\begin{array}{c}\text { Single/ } \\
\text { Multiple } \\
\text { (year) }^{b}\end{array}$ & $\begin{array}{c}\text { N ferti- } \\
\text { lizing }^{c} \\
\text { (kg N/ha) }\end{array}$ & $\begin{array}{c}\text { Biochar } \\
\text { added } \\
\text { (Mg/ha) }\end{array}$ & $\begin{array}{c}\text { Yield } \\
\text { (Mg/ha) }\end{array}$ & $\begin{array}{l}\text { Rel. } \\
\text { Yield }\end{array}$ & $\begin{array}{l}\text { Signifi- }^{\text {cance }^{d}} \\
\end{array}$ & $\begin{array}{c}\text { Yield } \\
\text { change }^{\mathrm{e}} \\
(\mathrm{Mg} / \mathrm{ha})\end{array}$ & $\begin{array}{c}\text { Revenue }^{\mathrm{e}} \\
\text { (USD/ } \\
\text { Mg BC) }\end{array}$ \\
\hline \multirow{5}{*}{$\begin{array}{l}35 \\
\text { cont'd }\end{array}$} & Ryegrass & $\mathrm{BC}+\mathrm{Ca}$ & $\mathrm{BC}($ manure $)$ & Y2 & 0 & 0 & 4.06 & 1.11 & n.s. & 0.42 & \\
\hline & Ryegrass & $\mathrm{BC}+\mathrm{Ca}$ & BC(slurry) & Y2 & 0 & 0 & 3.54 & 0.87 & n.s. & -0.11 & \\
\hline & Ryegrass & $\mathrm{MF}+\mathrm{Ca}$ & & Y2 & 368 & & 6.79 & & & & \\
\hline & Ryegrass & $\mathrm{MF}+\mathrm{BC}+\mathrm{Ca}$ & BC(manure) & Y2 & 368 & 0 & 8.50 & 1.25 & n.s. & 1.71 & \\
\hline & Ryegrass & $\mathrm{MF}+\mathrm{BC}+\mathrm{Ca}$ & BC(slurry) & Y2 & 368 & 0 & 7.49 & 1.10 & n.s. & 0.70 & \\
\hline \multirow[t]{16}{*}{36} & Wheat & $\mathrm{MF}$ & MF55 soluble & $\mathrm{S}$ & 15 & & 1.79 & & & & \\
\hline & Wheat & $\mathrm{MF}+\mathrm{BC}$ & MF55 soluble & $\mathrm{S}$ & 15 & 1.5 & 1.79 & 1.00 & n.s. & -0.01 & -0.32 \\
\hline & Wheat & $\mathrm{MF}$ & MF55 soluble & S & 15 & & 1.87 & & & & \\
\hline & Wheat & $\mathrm{MF}+\mathrm{BC}$ & MF55 soluble & $\mathrm{S}$ & 15 & 6.0 & 1.81 & 0.97 & n.s. & -0.06 & -0.99 \\
\hline & Wheat & $\mathrm{MF}$ & MF30 soluble & S & 13 & & 1.88 & & & & \\
\hline & Wheat & $\mathrm{MF}+\mathrm{BC}$ & MF30 soluble & $\mathrm{S}$ & 13 & 1.5 & 1.89 & 1.01 & n.s. & 0.01 & 0.63 \\
\hline & Wheat & $\mathrm{MF}$ & MF30 soluble & S & 13 & & 1.96 & & & & \\
\hline & Wheat & $\mathrm{MF}+\mathrm{BC}$ & MF30 soluble & $\mathrm{S}$ & 13 & 6.0 & 2.31 & 1.18 & $*$ & 0.34 & 5.43 \\
\hline & Wheat & $\mathrm{MF}$ & MF100 solid & S & 16 & & 1.35 & & & & \\
\hline & Wheat & $\mathrm{MF}+\mathrm{BC}$ & MF100 solid & S & 16 & 1.5 & 2.03 & 1.51 & $*$ & 0.68 & 43.07 \\
\hline & Wheat & $\mathrm{MF}$ & MF100 solid & S & 16 & & 1.41 & & & & \\
\hline & Wheat & $\mathrm{MF}+\mathrm{BC}$ & MF100 solid & S & 16 & 6.0 & 2.01 & 1.43 & $*$ & 0.60 & 9.51 \\
\hline & Wheat & $\mathrm{MF}$ & MF55 soluble & S & 12 & & 0.87 & & & & \\
\hline & Wheat & $\mathrm{MF}+\mathrm{BC}$ & MF55 soluble & $\mathrm{S}$ & 12 & 1.5 & 0.93 & 1.07 & $*$ & 0.06 & 3.60 \\
\hline & Wheat & $\mathrm{MF}$ & MF55 soluble & S & 12 & & 0.87 & & & & \\
\hline & Wheat & $\mathrm{MF}+\mathrm{BC}$ & MF55 soluble & $\mathrm{S}$ & 12 & 6.0 & 0.94 & 1.09 & $*$ & 0.08 & 1.20 \\
\hline \multirow[t]{4}{*}{37} & Sorghum & $\mathrm{MF}$ & First harvest & Y1 & 60 & & 0.28 & & & & \\
\hline & Sorghum & $\mathrm{MF}+\mathrm{BC}$ & First harvest & Y1 & 60 & 11.0 & 0.42 & 1.50 & n.s. & 0.14 & 0.81 \\
\hline & Sorghum & $\mathrm{MF}$ & Second harvest & Y2 & 55 & & 0.14 & & & & \\
\hline & Sorghum & $\mathrm{MF}+\mathrm{BC}$ & Second harvest & Y2 & 55 & 0 & 0.28 & 2.00 & n.s. & 0.14 & 0.81 \\
\hline \multirow[t]{4}{*}{38} & Lettuce & $\mathrm{MF}$ & & $\mathrm{S}$ & 113 & & 7.4 & & & & \\
\hline & Lettuce & $\mathrm{MF}+\mathrm{BC}$ & & $\mathrm{S}$ & 113 & 5.0 & 7.3 & 0.99 & n.s. & -0.10 & \\
\hline & Cover crop & $\mathrm{MF}$ & Winter cover crop & S & 0 & & 12.6 & & & & \\
\hline & Cover crop & $\mathrm{MF}+\mathrm{BC}$ & Winter cover crop & $\mathrm{S}$ & 0 & 10.0 & 11.3 & 0.90 & n.s. & -1.30 & \\
\hline
\end{tabular}




\begin{tabular}{|c|c|c|c|c|c|c|c|c|c|c|c|}
\hline $\begin{array}{l}\text { Ref. } \\
\text { No. }\end{array}$ & Crop & Variant $^{\mathrm{a}}$ & $\begin{array}{l}\text { Treatment, plot, site, } \\
\text { year }\end{array}$ & $\begin{array}{c}\text { Single/ } \\
\text { Multiple } \\
\text { (year) }^{b}\end{array}$ & $\begin{array}{c}\text { N ferti- } \\
\text { lizing }^{c} \\
\text { (kg N/ha) }\end{array}$ & $\begin{array}{c}\text { Biochar } \\
\text { added } \\
\text { (Mg/ha) }\end{array}$ & $\begin{array}{c}\text { Yield } \\
\text { (Mg/ha) }\end{array}$ & $\begin{array}{l}\text { Rel. } \\
\text { Yield }\end{array}$ & $\begin{array}{l}\text { Signifi- } \\
\text { cance }^{d}\end{array}$ & $\begin{array}{c}\text { Yield } \\
\text { change }^{\mathrm{e}} \\
(\mathrm{Mg} / \mathrm{ha})\end{array}$ & $\begin{array}{c}\text { Revenue }^{\mathrm{e}} \\
\text { (USD/ } \\
\text { Mg BC) }\end{array}$ \\
\hline \multirow{2}{*}{$\begin{array}{c}38 \\
\text { cont'd }\end{array}$} & lettuce & $\mathrm{MF}$ & & $\mathrm{S}$ & 113 & & 15.9 & & & & \\
\hline & lettuce & $\mathrm{MF}+\mathrm{BC}$ & & $\mathrm{S}$ & 113 & 10.0 & 9.3 & 0.58 & $*$ & -6.60 & \\
\hline \multirow[t]{6}{*}{39} & Maize & $\mathrm{MF}$ & Year 1 & Y1 & 135 & & 5.27 & & & & \\
\hline & Maize & $\mathrm{MF}+\mathrm{BC}$ & $\begin{array}{l}\text { Year 1; BC(coconut } \\
\text { shell) }\end{array}$ & Y1 & 135 & 15.0 & 5.71 & 1.08 & n.s. & 0.44 & 1.74 \\
\hline & Maize & $\mathrm{MF}+\mathrm{BC}$ & Year 1; BC(cattll dung) & Y1 & 135 & 15.0 & 5.81 & 1.10 & $*$ & 0.54 & 2.13 \\
\hline & & MF & Year 2 & Y2 & 135 & & 4.67 & & & & \\
\hline & Maize & $\mathrm{MF}+\mathrm{BC}$ & $\begin{array}{l}\text { Year 2; BC(coconut } \\
\text { shell) }\end{array}$ & Y2 & 135 & 0 & 5.65 & 1.21 & $*$ & 0.98 & 3.87 \\
\hline & Maize & $\mathrm{MF}+\mathrm{BC}$ & Year 2; BC(cattll dung) & Y2 & 135 & 0 & 5.87 & 1.26 & $*$ & 1.20 & 4.73 \\
\hline \multirow[t]{19}{*}{40} & Maize & $\mathrm{C}$ & 2010 & $\mathrm{Y} 1$ & 0 & & 6.67 & & & & \\
\hline & Wheat & B5 & 2010 & Y1 & 0 & 5 & 6.33 & 0.95 & n.s. & -0.34 & -6.44 \\
\hline & Wheat & B10 & 2010 & Y1 & 0 & 10 & 6.43 & 0.96 & n.s. & -0.24 & -2.27 \\
\hline & Wheat & $\mathrm{C}$ & 2011 & $\mathrm{Y} 2$ & 0 & & 6.58 & & & & \\
\hline & Wheat & B5 & 2011 & Y2 & 0 & 0 & 6.57 & 1.00 & n.s. & -0.01 & -0.19 \\
\hline & Wheat & B10 & 2011 & Y2 & 0 & 0 & 6.57 & 1.00 & n.s. & -0.01 & -0.09 \\
\hline & Wheat & $\mathrm{C}$ & 2012 & Y3 & 0 & & 6.07 & & & & \\
\hline & Wheat & B5 & 2012 & Y3 & 0 & 0 & 5.62 & 0.93 & n.s. & -0.45 & -8.53 \\
\hline & Wheat & B10 & 2012 & Y3 & 0 & 0 & 5.89 & 0.97 & n.s. & -0.18 & -1.71 \\
\hline & Rapeseed & $\mathrm{C}$ & 2010 & Y1 & 0 & & 1.2 & & & & \\
\hline & Rapeseed & B5 & 2010 & Y1 & 0 & 5 & 1.01 & 0.84 & n.s. & -0.19 & -21.47 \\
\hline & Rapeseed & B10 & 2010 & Y1 & 0 & 10 & 1.13 & 0.94 & n.s. & -0.07 & -3.96 \\
\hline & Rapeseed & $\mathrm{C}$ & 2011 & Y2 & 0 & & 1.05 & & & & \\
\hline & Rapeseed & B5 & 2011 & Y2 & 0 & 0 & 1.02 & 0.97 & n.s. & -0.03 & -3.39 \\
\hline & Rapeseed & B10 & 2011 & Y2 & 0 & 0 & 1.07 & 1.02 & n.s. & 0.02 & 1.13 \\
\hline & Rapeseed & $\mathrm{C}$ & 2012 & Y3 & 0 & & 0.76 & & & & \\
\hline & Rapeseed & B5 & 2012 & Y3 & 0 & 0 & 0.67 & 0.88 & n.s. & -0.09 & -10.17 \\
\hline & Rapeseed & B10 & 2012 & Y3 & 0 & 0 & 0.79 & 1.04 & n.s. & 0.03 & 1.70 \\
\hline & Vicia faba & $\mathrm{C}$ & 2010 & Y1 & 0 & & 3.32 & & & & \\
\hline
\end{tabular}




\begin{tabular}{|c|c|c|c|c|c|c|c|c|c|c|c|}
\hline $\begin{array}{l}\text { Ref. } \\
\text { No. }\end{array}$ & Crop & Variant $^{\mathbf{a}}$ & $\begin{array}{l}\text { Treatment, plot, site, } \\
\text { year }\end{array}$ & $\begin{array}{c}\text { Single/ } \\
\text { Multiple } \\
\text { (year) }^{b}\end{array}$ & $\begin{array}{c}\text { N ferti- } \\
\text { lizing }^{c} \\
(\mathrm{~kg} N / \mathbf{h a})\end{array}$ & $\begin{array}{c}\text { Biochar } \\
\text { added } \\
\text { (Mg/ha) }\end{array}$ & $\begin{array}{c}\text { Yield } \\
\text { (Mg/ha) }\end{array}$ & $\begin{array}{l}\text { Rel. } \\
\text { Yield }\end{array}$ & $\begin{array}{l}\text { Signifi- } \\
\text { cance }^{d}\end{array}$ & $\begin{array}{c}\text { Yield } \\
\text { change }^{\mathrm{e}} \\
(\mathrm{Mg} / \mathrm{ha})\end{array}$ & $\begin{array}{c}\text { Revenue }^{\mathrm{e}} \\
\text { (USD/ } \\
\text { Mg BC) }\end{array}$ \\
\hline \multirow{8}{*}{$\begin{array}{c}\mathbf{4 0} \\
\text { cont'd }\end{array}$} & Vicia faba & B5 & 2010 & Y1 & 0 & 5 & 3.27 & 0.98 & n.s. & -0.05 & -2.61 \\
\hline & Vicia faba & B10 & 2010 & Y1 & 0 & 10 & 3.28 & 0.99 & n.s. & -0.04 & -1.04 \\
\hline & Vicia faba & $\mathrm{C}$ & 2011 & Y2 & 0 & & 3.77 & & & & \\
\hline & Vicia faba & B5 & 2011 & Y2 & 0 & 0 & 3.87 & 1.03 & n.s. & 0.1 & 5.22 \\
\hline & Vicia faba & B10 & 2011 & Y2 & 0 & 0 & 3.83 & 1.02 & n.s. & 0.06 & 1.57 \\
\hline & Vicia faba & $\mathrm{C}$ & 2012 & Y3 & 0 & & 4.77 & & & & \\
\hline & Vicia faba & B5 & 2012 & Y3 & 0 & 0 & 4.40 & 0.92 & n.s. & -0.37 & -19.31 \\
\hline & Vicia faba & B10 & 2012 & Y3 & 0 & 0 & 4.66 & 0.98 & n.s. & -0.11 & -2.87 \\
\hline \multirow[t]{10}{*}{41} & Spring wheat & $\mathrm{C}$ & 2011 & Y1 & 0 & & 3.80 & & & & \\
\hline & Spring wheat & B5 & 2011 & Y1 & 0 & 5 & 3.50 & 0.92 & n.s. & -0.30 & -5.68 \\
\hline & Spring wheat & B10 & 2011 & Y1 & 0 & 10 & 3.60 & 0.95 & n.s. & -0.20 & -1.89 \\
\hline & Spring wheat & B20 & 2011 & Y1 & 0 & 20 & 3.40 & 0.89 & n.s. & -0.40 & -1.89 \\
\hline & Spring wheat & B30 & 2011 & Y1 & 0 & 30 & 4.10 & 1.08 & n.s. & 0.30 & 0.95 \\
\hline & Spring wheat & C & 2012 & $\mathrm{Y} 2$ & 0 & & 2.00 & & & & \\
\hline & Spring wheat & B5 & 2012 & Y2 & 0 & 0 & 1.90 & 0.95 & n.s. & -0.10 & -1.89 \\
\hline & Spring wheat & B10 & 2012 & Y2 & 0 & 0 & 1.80 & 0.90 & n.s. & -0.20 & -1.89 \\
\hline & Spring wheat & B20 & 2012 & Y2 & 0 & 0 & 1.60 & 0.80 & n.s. & -0.40 & -1.89 \\
\hline & Spring wheat & B30 & 2012 & $\mathrm{Y} 2$ & 0 & 0 & 1.90 & 0.95 & n.s. & -0.10 & -0.32 \\
\hline \multirow[t]{9}{*}{42} & Durum wheat & $\mathrm{MF}$ & Year 1 & Y1 & 122 & & 2.28 & & & & \\
\hline & Durum wheat & $\mathrm{MF}+\mathrm{BC}$ & Year 1 & Y1 & 122 & 30.0 & 2.92 & 1.28 & $*$ & 0.64 & 2.02 \\
\hline & Durum wheat & $\mathrm{MF}+\mathrm{BC}$ & Year 1 & Y1 & 122 & 60.0 & 2.93 & 1.29 & $*$ & 0.65 & 1.03 \\
\hline & Durum wheat & MF & Year 2 & Y2 & 122 & & 2.18 & & & & \\
\hline & Durum wheat & $\mathrm{MF}+\mathrm{BC}$ & Year 2 & Y2 & 122 & 0 & 2.90 & 1.33 & n.s. & 0.72 & 2.27 \\
\hline & Durum wheat & $\mathrm{MF}+\mathrm{BC}$ & Year 2 & Y2 & 122 & 0 & 2.71 & 1.24 & n.s. & 0.53 & 0.84 \\
\hline & Durum wheat & MF & $2^{\text {nd }}$ trial (single year) & $\mathrm{S}$ & 122 & & 2.40 & & & & \\
\hline & Durum wheat & $\mathrm{MF}+\mathrm{BC}$ & $2^{\text {nd }}$ trial (single year) & $\mathrm{S}$ & 122 & 30.0 & 3.19 & 1.33 & $*$ & 0.79 & 2.49 \\
\hline & Durum wheat & $\mathrm{MF}+\mathrm{BC}$ & $2^{\text {nd }}$ trial (single year) & $\mathrm{S}$ & 122 & 60.0 & 3.34 & 1.39 & $*$ & 0.94 & 1.48 \\
\hline \multirow{2}{*}{$\begin{array}{l}43 \\
(\mathrm{p})\end{array}$} & Tomato & MF & & S & 110 & & 88.33 & & & & \\
\hline & Tomato & $\mathrm{MF}+\mathrm{BC}$ & Slow pyrolysis & $\mathrm{S}$ & 110 & 14.0 & 86.20 & 0.98 & n.s. & -2.13 & \\
\hline
\end{tabular}




\begin{tabular}{|c|c|c|c|c|c|c|c|c|c|c|c|}
\hline $\begin{array}{l}\text { Ref. } \\
\text { No. }\end{array}$ & Crop & Variant $^{\mathrm{a}}$ & $\begin{array}{l}\text { Treatment, plot, site, } \\
\text { year }\end{array}$ & $\begin{array}{c}\text { Single/ } \\
\text { Multiple } \\
\text { (year) }^{b}\end{array}$ & $\begin{array}{c}\text { N ferti- } \\
\text { lizing }^{c} \\
\text { (kg N/ha) }\end{array}$ & $\begin{array}{c}\text { Biochar } \\
\text { added } \\
\text { (Mg/ha) }\end{array}$ & $\begin{array}{c}\text { Yield } \\
\text { (Mg/ha) }\end{array}$ & $\begin{array}{l}\text { Rel. } \\
\text { Yield }\end{array}$ & $\begin{array}{l}\text { Signifi- } \\
\text { cance }^{d}\end{array}$ & $\begin{array}{c}\text { Yield } \\
\text { change }^{\mathrm{e}} \\
(\mathrm{Mg} / \mathrm{ha})\end{array}$ & $\begin{array}{c}\text { Revenue }^{\mathrm{e}} \\
\text { (USD/ } \\
\text { Mg BC) }\end{array}$ \\
\hline & Tomato & $\mathrm{MF}+\mathrm{BC}$ & Fast pyrolysis & $\mathrm{S}$ & 110 & 14.0 & 92.29 & 1.04 & n.s. & 3.96 & \\
\hline \multirow{16}{*}{$\begin{array}{l}44 \\
(q)\end{array}$} & Maize & $\mathrm{MF}$ & Field A & $S$ & 75 & & 7.55 & & & & \\
\hline & Maize & $\mathrm{MF}+\mathrm{BC}$ & Field A & S & 75 & 10.0 & 14.71 & 1.95 & $*$ & 7.16 & \\
\hline & Cowpea & $\mathrm{MF}$ & Field A & S & 75 & & 12.04 & & & & \\
\hline & Cowpea & $\mathrm{MF}+\mathrm{BC}$ & Field A & S & 75 & 10.0 & 13.76 & 1.14 & n.s. & 1.72 & \\
\hline & Groundnut & $\mathrm{MF}$ & Field A & S & 75 & & 4.30 & & & & \\
\hline & Groundnut & $\mathrm{MF}+\mathrm{BC}$ & Field A & $\mathrm{S}$ & 75 & 10.0 & 5.76 & 1.34 & $*$ & 1.46 & \\
\hline & Maize & $\mathrm{MF}$ & Field B & S & 75 & & 10.45 & & & & \\
\hline & Maize & $\mathrm{MF}+\mathrm{BC}$ & Field B & S & 75 & 10.0 & 10.06 & 0.96 & n.s. & -0.39 & \\
\hline & Cowpea & $\mathrm{MF}$ & Field B & S & 75 & & 11.35 & & & & \\
\hline & Cowpea & $\mathrm{MF}+\mathrm{BC}$ & Field B & S & 75 & 10.0 & 12.56 & 1.11 & n.s. & 1.21 & \\
\hline & Groundnut & $\mathrm{MF}$ & Field B & S & 75 & & 5.85 & & & & \\
\hline & Groundnut & $\mathrm{MF}+\mathrm{BC}$ & Field B & S & 75 & 10.0 & 6.54 & 1.12 & n.s. & 0.69 & \\
\hline & Maize & $\mathrm{MF}$ & Field C & S & 75 & & 10.36 & & & & \\
\hline & Maize & $\mathrm{MF}+\mathrm{BC}$ & Field C & $\mathrm{S}$ & 75 & 10.0 & 15.30 & 1.48 & $*$ & 4.94 & \\
\hline & Maize & $\mathrm{C}$ & Field C & S & 0 & & 9.23 & & & & \\
\hline & Maize & $\mathrm{BC}$ & Field C & $\mathrm{S}$ & 0 & 10.0 & 11.50 & 1.25 & $*$ & 2.27 & \\
\hline \multirow{12}{*}{$\begin{array}{l}\mathbf{4 5} \\
(\mathrm{r})\end{array}$} & Maize & $\mathrm{MF}$ & & $S$ & yes & & 10.50 & & & & \\
\hline & Maize & $\mathrm{MF}+\mathrm{BC}$ & BC(maize stalk) & S & yes & 2.0 & 12.18 & 1.16 & n.s. & 1.68 & 49.70 \\
\hline & Maize & $\mathrm{MF}+\mathrm{BC}$ & BC(maize stalk) & $S$ & yes & 4.0 & 12.60 & 1.20 & n.s. & 2.10 & 31.06 \\
\hline & Maize & $\mathrm{MF}+\mathrm{BC}$ & $\mathrm{BC}$ (rice straw) & $\mathrm{S}$ & yes & 2.0 & 12.36 & 1.18 & n.s. & 1.86 & 55.03 \\
\hline & Maize & $\mathrm{MF}+\mathrm{BC}$ & $\mathrm{BC}$ (rice straw) & S & yes & 4.0 & 12.96 & 1.23 & n.s. & 2.46 & 36.39 \\
\hline & Peanut & $\mathrm{MF}$ & & $\mathrm{S}$ & yes & & 4.20 & & & & \\
\hline & Peanut & $\mathrm{MF}+\mathrm{BC}$ & BC(maize stalk) & $S$ & yes & 2.0 & 4.68 & 1.11 & n.s. & 0.48 & 113.76 \\
\hline & Peanut & $\mathrm{MF}+\mathrm{BC}$ & $\mathrm{BC}($ maize stalk $)$ & $\mathrm{S}$ & yes & 4.0 & 5.10 & 1.21 & n.s. & 0.90 & 106.65 \\
\hline & Peanut & $\mathrm{MF}+\mathrm{BC}$ & $\mathrm{BC}$ (rice straw) & S & yes & 2.0 & 4.98 & 1.19 & n.s. & 0.78 & 184.86 \\
\hline & Peanut & $\mathrm{MF}+\mathrm{BC}$ & $\mathrm{BC}$ (rice straw) & $\mathrm{S}$ & yes & 4.0 & 5.22 & 1.24 & n.s. & 1.02 & 120.87 \\
\hline & Sweet potato & $\mathrm{MF}$ & & $\mathrm{S}$ & yes & & 33.0 & & & & \\
\hline & Sweet potato & $\mathrm{MF}+\mathrm{BC}$ & BC(maize stalk) & $\mathrm{S}$ & yes & 1.0 & 39.6 & 1.20 & n.s. & 6.60 & \\
\hline
\end{tabular}




\begin{tabular}{|c|c|c|c|c|c|c|c|c|c|c|c|}
\hline $\begin{array}{l}\text { Ref. } \\
\text { No. }\end{array}$ & Crop & Variant $^{\mathrm{a}}$ & $\begin{array}{l}\text { Treatment, plot, site, } \\
\text { year }\end{array}$ & $\begin{array}{c}\text { Single/ } \\
\text { Multiple } \\
\text { (year) }^{b}\end{array}$ & $\begin{array}{c}\text { N ferti- } \\
\text { lizing }^{c} \\
\text { (kg N/ha) }\end{array}$ & $\begin{array}{c}\text { Biochar } \\
\text { added } \\
\text { (Mg/ha) }\end{array}$ & $\begin{array}{c}\text { Yield } \\
\text { (Mg/ha) }\end{array}$ & $\begin{array}{l}\text { Rel. } \\
\text { Yield }\end{array}$ & $\begin{array}{l}\text { Signifi- } \\
\text { cance }^{d}\end{array}$ & $\begin{array}{c}\text { Yield } \\
\text { change }^{\mathrm{e}} \\
(\mathrm{Mg} / \mathrm{ha})\end{array}$ & $\begin{array}{c}\text { Revenue }^{\mathrm{e}} \\
\text { (USD/ } \\
\text { Mg BC) }\end{array}$ \\
\hline \multirow{7}{*}{$\begin{array}{c}45 \\
\text { cont'd }\end{array}$} & Sweet potato & $\mathrm{MF}+\mathrm{BC}$ & $\mathrm{BC}($ rice straw) & $\mathrm{S}$ & yes & 2.0 & 37.6 & 1.14 & n.s. & 4.62 & \\
\hline & Sweet potato & $\mathrm{MF}+\mathrm{BC}$ & $\mathrm{BC}$ (rice straw) & S & yes & 4.0 & 38.9 & 1.18 & n.s. & 5.94 & \\
\hline & Wheat & $\mathrm{MF}$ & & $\mathrm{S}$ & yes & & 7.16 & & & & \\
\hline & Wheat & $\mathrm{MF}+\mathrm{BC}$ & BC(maize stalk) & $\mathrm{S}$ & yes & 2.0 & 8.60 & 1.20 & n.s. & 1.44 & 68.21 \\
\hline & Wheat & $\mathrm{MF}+\mathrm{BC}$ & BC(maize stalk) & S & yes & 4.0 & 6.90 & 0.96 & n.s. & -0.26 & -6.16 \\
\hline & Wheat & $\mathrm{MF}+\mathrm{BC}$ & $\mathrm{BC}$ (rice straw) & $S$ & yes & 2.0 & 7.70 & 1.08 & n.s. & 0.54 & 25.58 \\
\hline & Wheat & $\mathrm{MF}+\mathrm{BC}$ & $\mathrm{BC}$ (rice straw) & S & yes & 4.0 & 9.00 & 1.26 & n.s. & 1.84 & 43.58 \\
\hline \multirow[t]{6}{*}{46} & Rice & $\mathrm{C}$ & & $\mathrm{S}$ & 0 & & 8.6 & & & & \\
\hline & Rice & $\mathrm{BC}$ & BC10 & S & 0 & 10.0 & 9.6 & 1.12 & $*$ & 1.00 & 33.00 \\
\hline & Rice & $\mathrm{BC}$ & BC40 & S & 0 & 40.0 & 9.8 & 1.14 & $*$ & 1.20 & 9.90 \\
\hline & Rice & $\mathrm{MF}$ & & S & 300 & & 9.1 & & & & \\
\hline & Rice & $\mathrm{MF}+\mathrm{BC}$ & BC10 & S & 300 & 10.0 & 9.9 & 1.09 & $*$ & 0.80 & 26.40 \\
\hline & Rice & $\mathrm{MF}+\mathrm{BC}$ & BC40 & S & 300 & 40.0 & 10.2 & 1.12 & $*$ & 1.10 & 9.08 \\
\hline \multirow[t]{6}{*}{47} & Rice & $\mathrm{C}$ & & $S$ & 0 & & 6.28 & & & & \\
\hline & Rice & $\mathrm{BC}$ & BC20 & S & 0 & 20.0 & 7.27 & 1.16 & $*$ & 0.99 & 2.93 \\
\hline & Rice & $\mathrm{BC}$ & BC40 & $S$ & 0 & 40.0 & 6.74 & 1.07 & $*$ & 0.46 & 0.68 \\
\hline & Rice & $\mathrm{MF}$ & & S & 300 & & 6.65 & & & & \\
\hline & Rice & $\mathrm{MF}+\mathrm{BC}$ & BC20 & $S$ & 300 & 20.0 & 7.86 & 1.18 & $*$ & 1.21 & 3.58 \\
\hline & Rice & $\mathrm{MF}+\mathrm{BC}$ & BC40 & $S$ & 300 & 40.0 & 7.42 & 1.12 & $*$ & 0.77 & 1.14 \\
\hline \multirow[t]{8}{*}{48} & Rice & $\mathrm{MF}$ & Year 1 & Y1 & 300 & & 7.6 & & & & \\
\hline & Rice & $\mathrm{MF}+\mathrm{BC}$ & Year 1 & Y1 & 300 & 10.0 & 8.3 & 1.09 & n.s. & 0.70 & 23.10 \\
\hline & Rice & $\mathrm{MF}+\mathrm{BC}$ & Year 1 & Y1 & 300 & 20.0 & 8.4 & 1.11 & n.s. & 0.80 & 13.20 \\
\hline & Rice & $\mathrm{MF}+\mathrm{BC}$ & Year 1 & Y1 & 300 & 40.0 & 8.5 & 1.12 & n.s. & 0.90 & 7.43 \\
\hline & Rice & $\mathrm{MF}$ & Year 2 & Y2 & 300 & & 7.6 & & & & \\
\hline & Rice & $\mathrm{MF}+\mathrm{BC}$ & Year 2 & Y2 & 300 & 0 & 9.7 & 1.28 & $*$ & 2.10 & 69.30 \\
\hline & Rice & $\mathrm{MF}+\mathrm{BC}$ & Year 2 & Y2 & 300 & 0 & 8.3 & 1.09 & n.s. & 0.70 & 11.55 \\
\hline & Rice & $\mathrm{MF}+\mathrm{BC}$ & Year 2 & Y2 & 300 & 0 & 9.3 & 1.22 & $*$ & 1.70 & 14.03 \\
\hline
\end{tabular}


Grey shaded lines: variants without biochar added, serve as reference value for calculation of relative yield and yield difference of variants with biochar added in following (white) lines

a) C: control (no treatment), BC: biochar, MF: mineral fertilizer (nitrogen amount see coloum N fertilizing), PF: phosphate fertilizer, Man: manure, Com: compost, Dig: digestate, Mic: inoculted with microbes.

b) S: singe year result; Y1, Y2, Y3, Y4, Y5: no. of year of multi-year results.

$\left.{ }^{c}\right)$ Amount of mineral nitrogen fertilizer applied.

d) Significance according to original publication, *: significant (various p-levels), n.s.: not significant.

e) Absolute yield change and revenue calculated only for crops with a market price. Revenue $=($ Yield change * market price) / Biochar added; market prices ref. Table S3

f) BC quantities "whole plot rates" used.

$\left.{ }^{g}\right)$ Pot experiment under field conditions; results for biochar applicationrate of $300 \mathrm{Mg} / \mathrm{ha}$ not respected .

h) Planted in wholes, areal biochar application amounts to 0.8 and 4 t/ha, respectively, which "corresponds to 6 and 30 t/ha in conventional farming“" (Cornelissen et al., 2013).

i) Variant "annually $1 \mathrm{t}$ biochar" not accounted.

$\left.{ }^{k}\right)$ For calculation of multiple-year revenue only data for main crops respected.

1) Mineral nitrogen fertilizing amount not specified for some sites: "Usual application of fertilizer in the agricultural context of normal farm practice" (Hammond et al., 2013); two results not respected due to an untypically very low yield of control plot.

$\mathrm{m}$ ) Yield results related to hectare by the authors.

n) No harvest results in year 3 .

$\left.{ }^{\circ}\right)$ Data taken from Fig. 2; biochar was added in the season before the field study started.

p) Tomato fresh fruit marketable produktion

q) No information given on harvested plant matter (grain, fresh matter).

${ }^{\mathrm{r}}$ ) Mineral fertilizer added to all variants, but quantity not specified. 


\section{References to Table S1}

1 Abukari A. Effect of rice husk biochar on maize productivity in the Guinea savannah zone of Ghana. MSc-Thesis, Faculty Renewable Natural Resources, Univ. Science and Technology, Kumasi, Ghana, 116 p (2014).

2 Agegnehu G, Bass AM, Nelson PN, Bird MI. Benefit of biochar, compost and biocharcompost for soil quality, maize yield and greenhouse gas emissions in a tropical agricultural soil. Science Total Environment 543, 295-306 (2016).

3 Arif M, Ali A, Umair M, Munsif F, Ali K, Inamullah MS, Ayub G. Effect of biochar FYM and mineral nitrogen alone and in combination on yield and yield components of maize. Sarhad Journal Agriculture 28, 191-195 (2012).

4 Asai H, Samson BK, Stephan HM, Songyikhangsuthor K et al. Biochar amendment techniques for upland rice production in Northern Laos: 1. Soil physical properties, leaf SPAD and grain yield. Field Crops Research 111, 81-84 (2009).

5 Baronti S, Alberti G, Delle Vedove G, Di Gennaro F, Fellet G et al. The biochar option to improve plant yields: first results from some field and pot experiments in Italy. Italian Journal Agronomy 5, 3-11 (2010).

6 Bass AM, Bird MI, Kay G, Muirhead B. Soil properties, greenhouse gas emissions and crop yield under compost, biochar and co-composted biochar in two tropical agronomic systems. Science Total Environment 550, 459-470 (2016),

7 Blackwell P, Krull E, Butler G, Herbert A, Solaiman Z. Effect of banded biochar on dryland wheat production and fertiliser use in south-western Australia: an agronomic and economic perspective. Australian J. Soil Res. 48, 531-545 (2010).

8 Borchard N, Siemens J, Ladd B, Möller A, Amelung W. Application of biochars to sandy and silty soil failed to increase maize yield under common agricultural practice. Soil Tillage Research 144, 184-194 (2014).

9 Cornelissen G, Martinsen V, Shitumbanuma V, Alling V et al. Biochar effect on maize yield and soil characteristics in five conservation farming sites in Zambia. Agronomy 3, 256-274 (2013).

10 Cui L, Pan G, Li L, Yan J, Zhang A, Bian B, Chang R. The reduction of wheat Cd uptake in contaminated soil via biochar amendment: A two-year field experiment. BioResources 7(4), 5666-5676 (2012).

11 Curaqueo G, Meier S, Khan N, Cea M, Navia R. Use of biochar on two volcanic soils: effects on soil properties and barley yield. J. Soil Science Plant Nutrition 14(4), 911-924 (2014).

12 Farell M, Macdonald LM, Butler G, Chirino-Vallee I, Condron LM. Biochar and fertilizer application influence phosphorus fractionation and wheat yield. Biol Fertil Soils 50, 169-178 (2014).

13 Gajić A, Koch KH. Sugar Beet (Beta vulgaris L.) Growth Reduction Caused by Hydrochar Is Related to Nitrogen Supply. Journal Environmental Quality 41, 1067-1075 (2012).

14 Gaskin JW, Speir RA, Harris K, Das KC et al. Effect of Peanut Hull and Pine Chip Biochar onSoil Nutrients, Corn Nutrient Status, and Yield. Agronomy Journal 102(2), 623-633 (2010). 
15 Glaser B, Wiedner K, Seelig S, Schmidt HP, Gerber H. Biochar organic fertilizers from natural resources as substitute for mineral fertilizers. Agron. Sustain. Dev. 35, 667-678 (2015).

16 Golika B, Baskar K. Influence of biochar as a soil amendment on yield and quality of maize in alfisol of Thoothukudi district of Tamilnadu, India. Internat. J. Plant Animal Environm. Sci. 5(1), 152-155 (2015).

17 Güereña D, Lehmann J, Hanley K, Enders A, Hyland C, Riha S. Nitrogen dynamics following field application of biochar in a temperate North American maize-based production system. Plant Soil 365(1-2), 239-254 (2013).

18 Haefele SM, Konboon Y, Wongboon W, Amarante S, Maarifat AA, Pfeiffer EM, Knoblauch C. Effects and fate of biochar from rice residues in rice-based systems. Field Crops Research 121(3), 430-440 (2011).

19 Hall DJW, Bell RW. Biochar and Compost Increase Crop Yield but the Effect is Short Term on Sandplain Soils of Western Australia. Pedosphere 25(5), 720-728 (2015).

20 Hammond J, Shackley S, Prendergast-Millera M, Cooka J, Buckinghamb S, Pappab VA. Biochar field testing in the UK: outcomes and implications for use. Carbon Management 4(2), 159-170 (2013).

21 Islami T, Guritno B, Basuki N, Suryanto Islami A. Maize yield and associated soil quality changes in cassava+ maize intercropping system after 3 years of biochar application. Journal Agriculture Food Technology 1(7), 112-115 (2011).

22 Jay CN, Fitzgerald JD, Hipps NA, Atkinson CJ. Why short-term biochar application has no yield benefits: evidence from three field-grown crops. Soil Use Management 31, 241 250 (2015).

23 Jones DL, Rousk J, Edwards-Jones G, DeLuca TH, Murphy DV. Biochar-mediated changes in soil quality and plant growth in a three year field trial. Soil Biology Biochemistry 327, 235-246 (2012).

24 Karer J, Wimmer B, Zehetner F, Kloss S, Soja G. Biochar. application to temperate soils: effects on nutrient uptake and crop yield under field conditions. Agricultural Food Science 22(4), 390-403 (2013).

25 Kimetu JM, Lehmann J, Ngoze SO, Mugendi DN et al. Reversibility of Soil Productivity Decline with Organic Matter of Differing Quality Along a Degradation Gradient. Ecosystems 11, 726-739 (2008).

26 Lentz RD, Ippolito JA. Biochar and manure affect calcareous soil and Maize silage nutrient concentrations and uptake. Journal Environmental Quality 41(4), 1033-1043 (2012).

27 Liu XY, Qu JJ, Li LQ, Zhang AF et al. Can biochar amendment be an ecological engineering technology to depress $\mathrm{N}_{2} \mathrm{O}$ emissions in rice paddies? - A cross site field experiment from South China. Ecological Engineering 42, 168-173 (2012).

28 Liu X, Ye Y, Liu Y, Zhang A, Zhang X et al. Sustainable biochar effects for low carbon crop production: A 5-crop season field experiment on a low fertility soil from Central China. Agricultural Systems 129, 22-29 (2014a).

29 Liu Z, Chen X, Jing Y, Li Q, Zhang J, Huang Q. Effect of biochar amendment on rapeseed and sweet potato yields and water stable aggregates in upland red soil. Catena $123,45-51(2014 b)$ 
30 Major J, Rondon M, Molina D, Riha SJ, Lehmann J. Maize yield and nutrition during 4 years after biochar application to a Colombian savanna oxisol. Plant Soil 333(1), 117128 (2010).

31 Mokry M. CarboSolum - Schlussbericht. LTZ Augustenberg, Karlsruhe, Germany, 20 p. (2015).

32 Nehls T. Fertility improvement of a Terra Firme Oxisol in central Amazonia by charcoal applications. Final thesis, Geoecology, University of Bayreuth, Germany (2002).

33 Nellisen V, Ruysschaert G, Abusie DM, D'Hose T et al. Impact of woody biochar on properties of a sandy loam soil and spring barley during a two-year field experiment. European Journal Agronomy 62, 65-78 (2015).

34 Rogovska N, Laird DA, Rathke SJ, Karlen DL. Biochar impact on Midwestern Mollisols and maize nutrient availability. Geoderma 230-231, 340-347 (2014).

35 Sinclair K, Slavich P, Van Zwieten L, Downie A. Productivity and nutrient availability on a ferrosol: Biochar, lime and fertiliser. Proceedings of the 24th Annual Conference of the Grassland Society of NSW, 119-122 (2008) http://grasslandnsw.com.au/news/wpcontent/uploads/2009/10/Sinclair-Slavich-van-Zweiten-Downie-2009.pdf (2015).

36 Solaiman Z, Blackwell P, Abbott L, Storer P. Direct and residual effect of biochar application on mycorrhizal root colonization, growth and nutrition of wheat. Australian $J$. Soil Res. 48, 546-554 (2010).

37 Steiner C, Teixeira GW, Lehmann J, Nehls T, de Macêdo JLV, Blum WEH, Zech W. Long term effects of manure, charcoal and mineral fertilization on crop production and fertility on a highly weathered Central Amazonian upland soil. Plant Soil 291(1), 275290 (2007).

38 Suddick E, Six J. An estimation of annual nitrous oxide rmissions and soil quality following the amendment of high temperature walnut shell biochar and compost to a small scale vegetable crop rotation. Science Total Environment 465, 298-307 (2013).

39 Sukartono WHU, Kusuma Z, Nugroho WH (2011) Soil fertility status, nutrient uptake, and maize (Zea mays L.) yield following biochar and cattle manure application on sandy soils of Lombok, Indonesia. Journal Tropical Agriculture 49(1-2), 47-52.

40 Tammeorg P, Simojoki A, Mäkelä P, Stoddard FL, Alakukku L, Helenius J. Short-term effects of biochar on soil properties and wheat yield formation with meat bone meal and inorganic fertiliser on a boreal loamy sand. Agriculture Ecosystems Environment 191, 108-116 (2014).

41 Tammeorg P, Simojoki A, Mäkelä P, Stoddard FL, Alakukku L, Helenius J. Biochar application to a fertile sandy clay loam in boreal conditions: effects on soil properties and yield formation of wheat, turnip rape and faba bean. Plant Soil 374(1-2), 89-107 (2014).

42 Vaccari FP, Baronti S, Lugato E, Genesio L, Castaldi S, Fornasier F, Miglietta F. Biochar as a strategy to sequester carbon and increase yield in durum wheat. European Journal Agronomy 34(4), 231-238 (2011).

43 Vaccari FP,Maienza A, Miglietta F, Baronti S et al. Biochar stimulates plant growth but not fruit yield of processing tomato in fertile soil. Agriculture, Ecosystems Environment 207, 163-170 (2015). 
44 Yamato M, Okimori Y, Wibowo IF, Anshori S, Ogawa M. Effects of the application of charred bark of Acacia mangium on the yield of maize, cowpea and peanut, and soil chemical properties in South Sumatra, Indonesia. Soil Science Plant Nutrition 52(4), 489-495 (2006).

45 Yang Y, Ma S, Zhao Y, Jing M, Xu Y, Chen J. A Field Experiment of Enhancement of Crop Yield by Rice Straw and Maize Stalk-Derived Biochar in Northern China. Sustainability 7, 13713-13725 (2015).

46 Zhang A, Cui L, Pan G, Li L, Hussain Q, Zhang X, Zheng J, Crowley D. Effect of biochar amendment on yield and methane and nitrous oxide emissions from a rice paddy from Tai Lake plain, China. Agriculture Ecosystems Environment 139(4), 469-475 (2010).

47 Zhang A, Liu Y, Pan G, Hussain Q, Li L, Zheng J, Zhang X. Effect of biochar amendment on maize yield and greenhouse gas emissions from a soil organic carbon poor calcerous loamy sand from Central China Plain. Plant Soil 351, 263-275 (2012).

48 Zhang A, Bian R, Pan G, Cui L, Hussain Q, Li L, Zheng J et al. Effects of biochar amendment on soil quality, crop yield and greenhouse gas emission in a Chinese rice paddy: A field study of 2 consecutive rice growing cycles. Field Crops Research 127, 153-160 (2012). 
Table S2. Quantiles of absolute and relative change in crop yield (data of Table S1) from field experiments of biochar application to agricultural crops.

\begin{tabular}{|c|c|c|c|c|c|c|}
\hline \multirow[t]{2}{*}{ Crop } & \multicolumn{5}{|c|}{ Quantile } & \multirow[t]{2}{*}{$\mathbf{n}$} \\
\hline & $5 \%$ & $25 \%$ & $50 \%$ & $75 \%$ & $95 \%$ & \\
\hline & \multicolumn{5}{|c|}{----- Relative yield change $(-)^{\mathrm{a}}$} & \\
\hline Maize $^{c}$ & 0.761 & 0.968 & 1.075 & 1.290 & 2.203 & 168 \\
\hline Cereals $^{\mathrm{c}, \mathrm{d}}$ & 0.828 & 0.949 & 1.013 & 1.090 & 1.393 & 135 \\
\hline Rice $^{c}$ & 0.717 & 0.964 & 1.037 & 1.133 & 1.320 & 81 \\
\hline Other crops ${ }^{\mathrm{e}}$ & 0.598 & 0.944 & 1.028 & 1.202 & 1.782 & 123 \\
\hline \multirow[t]{2}{*}{ All crops } & 0.718 & 0.950 & 1.028 & 1.177 & 1.705 & 507 \\
\hline & \multicolumn{5}{|c|}{-- } & \\
\hline Maize $^{c}$ & -1.81 & -0.33 & 0.34 & 1.20 & 2.60 & 168 \\
\hline Cereals $^{\mathrm{c}, \mathrm{d}}$ & -0.48 & -0.14 & 0.04 & 0.26 & 0.74 & 135 \\
\hline Rice $^{c}$ & -0.80 & -0.12 & 0.10 & 0.50 & 1.00 & 81 \\
\hline Other crops ${ }^{\mathrm{f}}$ & -8.42 & -0.20 & 0.29 & 1.21 & 5.14 & 117 \\
\hline
\end{tabular}

a) Yield of variant 'with biochar' relative to yield of control (without biochar).

b) Yield of variant 'with biochar' minus yield of control (without biochar).

$\left.{ }^{c}\right)$ Yield of maize, cereals (not including maize), and rice is given as grain yield.

d) All other cereals not including maize.

e) Other crop: crop type is specified in Table S1.

f) Quantiles of absolute yield change for 'other crops' should be interpreted carefully due to the varying harvested crop parts as well as figures given for dry and fresh matter yield, respectively, for different crops. 
Table S3. Crop market prices used for calculation of revenue.

\begin{tabular}{lcl}
\hline Crop & $\begin{array}{c}\text { Market price } \\
\text { USD t }\end{array}$ & Source \\
\hline Corn & 59 & U.S. crop farm prices 2014/15 \\
Wheat, winter & 95 & U.S. crop farm prices 2014/15 \\
Rice, medium & 330 & U.S. crop farm prices 2014/15 \\
Hirse & 64 & U.S. crop farm prices 2014/15 \\
Gerste & 85 & U.S. crop farm prices 2014/15 \\
Rapeseed & 523 & U.S. crop farm prices 2014/15 \\
Groundnut & 474 & U.S. crop farm prices 2014/15 \\
Dry peas & 261 & U.S. crop farm prices 2014/15 \\
Sunflower seed & 456 & U.S. crop farm prices 2014/15 \\
Potatoes & 181 & www.usda.gov/nass/PUBS/TODAYRPT/agpr1215.pdf \\
Lupin grain & 200 & www.igrain.com.au/ \\
Sugar beet & 33,4 & Notation Germany 2015 \\
\hline
\end{tabular}

\section{References for Table S3}

April 2015 U.S. Crop Price Update. FAPRI-MU Bulletin \#08-15. Food and Agricultural Policy Research Institute (FAPRI), University of Missouri (MU), Columbia, MO.

Notation Germany 2015; www.liz-online.de/meldungen/alle/datum/2015/02/25/

die-ruebenpreise-2014-2015-stehen-fest.html (exchange rate USD / EURO = 1.10) 
Table S4. Median and quantiles of the revenue of biochar application to soils (data of Table S1).

\begin{tabular}{|c|c|c|c|c|c|c|}
\hline \multirow[t]{2}{*}{ Crop } & \multicolumn{5}{|c|}{ Quantile } & \multirow[t]{2}{*}{$\mathbf{n}$} \\
\hline & $5 \%$ & $25 \%$ & $\mathbf{5 0 \%}$ & $75 \%$ & $95 \%$ & \\
\hline & \multicolumn{5}{|c|}{------------- Revenue (USD/Mg biochar) ${ }^{\mathbf{c}}$----------- } & \\
\hline Maize $^{a}$ & -4.64 & 1.22 & 9.76 & 20.54 & 35.77 & 51 \\
\hline Cereals $^{\mathrm{a}, \mathrm{d}}$ & -98.53 & -0.79 & 2.99 & 10.33 & 45.57 & 54 \\
\hline Rice $^{\mathrm{a}}$ & -87.41 & -8.25 & 0.50 & 12.38 & 33.00 & 53 \\
\hline Other crops ${ }^{\mathrm{a}}$ & -301.02 & -193.14 & 10.80 & 60.67 & 123.08 & 21 \\
\hline Multi-year results ${ }^{b}$ & -7.62 & -1.89 & 0.32 & 2.76 & 11.39 & 93 \\
\hline All results & -93.43 & -1.77 & 1.57 & 10.74 & 44.76 & 272 \\
\hline
\end{tabular}

a) Single year results.

b) 2-5 year results, revenue averaged.

c) Note: Revenues are calculated as annual value, for complete evaluation on biochar profitability the annual revenue has to be multiplied by the expected number of years of biochar effectiveness.

d) Cereals not including maize. 
Table S5. Half-lifes of biochars from soil incubation experiments.

\begin{tabular}{|c|c|c|c|c|c|c|}
\hline $\begin{array}{l}\text { Ref. } \\
\text { No. }\end{array}$ & $\begin{array}{l}\text { Conversion method, highest } \\
\text { treatment temperature, feedstock }\end{array}$ & Variants & Factors & $\begin{array}{l}\text { Measuring } \\
\text { period }(d)\end{array}$ & $\begin{array}{l}\text { Half life } \\
\text { time (yr) }\end{array}$ & Note \\
\hline \multicolumn{7}{|c|}{ Pyrolytic or thermally produced biochars } \\
\hline \multirow[t]{2}{*}{1} & Pyrolysis, $575^{\circ} \mathrm{C}$, & Sandy soil & & 200 & $>1000$ & $\mathrm{a}$ \\
\hline & Miscanthus & Loamy sand & & 200 & 16.8 & $\mathrm{a}$ \\
\hline \multirow[t]{4}{*}{2} & Pyrolysis, PYREG, $650{ }^{\circ} \mathrm{C}$, & Sandy soil & & 196 & 51.7 & $\mathrm{a}$ \\
\hline & Miscanthus & Loamy sand & & 196 & 58.9 & $\mathrm{a}$ \\
\hline & Pyrolysis, UK BRC, $500{ }^{\circ} \mathrm{C}$, & Sandy soil & & 196 & 12.7 & $\mathrm{a}$ \\
\hline & Miscanthus & Loamy sand & & 196 & 10.1 & $\mathrm{a}$ \\
\hline \multirow[t]{2}{*}{3} & Pyrolysis Centrifuge & $\mathrm{T}=475^{\circ} \mathrm{C}$ & & 115 & 1.7 & $\mathrm{~h}$ \\
\hline & Reactor (PCR), & $\mathrm{T}=500^{\circ} \mathrm{C}$ & & 115 & 2.8 & $\mathrm{~h}$ \\
\hline 4 & two feedstocks & Poultry manure & & 60 & 1.7 & \\
\hline \multirow[t]{6}{*}{6} & Slow Pyrolysis, & Inceptosol & $\mathrm{T}=450^{\circ} \mathrm{C}$ & 730 & 91 & $\mathrm{~b}, \mathrm{c}$ \\
\hline & two temperatures, & & $\mathrm{T}=550^{\circ} \mathrm{C}$ & 730 & 505 & $\mathrm{~b}, \mathrm{c}$ \\
\hline & Woody biomass & Entisol & $\mathrm{T}=450^{\circ} \mathrm{C}$ & 730 & 0.3 & $\mathrm{~b}, \mathrm{c}$ \\
\hline & (Eucalyptus) & & $\mathrm{T}=550^{\circ} \mathrm{C}$ & 730 & 358 & $\mathrm{~b}, \mathrm{c}$ \\
\hline & & Oxisol & $\mathrm{T}=450^{\circ} \mathrm{C}$ & 730 & 0.3 & $\mathrm{~b}, \mathrm{c}$ \\
\hline & & & $\mathrm{T}=550^{\circ} \mathrm{C}$ & 730 & 330 & $\mathrm{~b}, \mathrm{c}$ \\
\hline
\end{tabular}




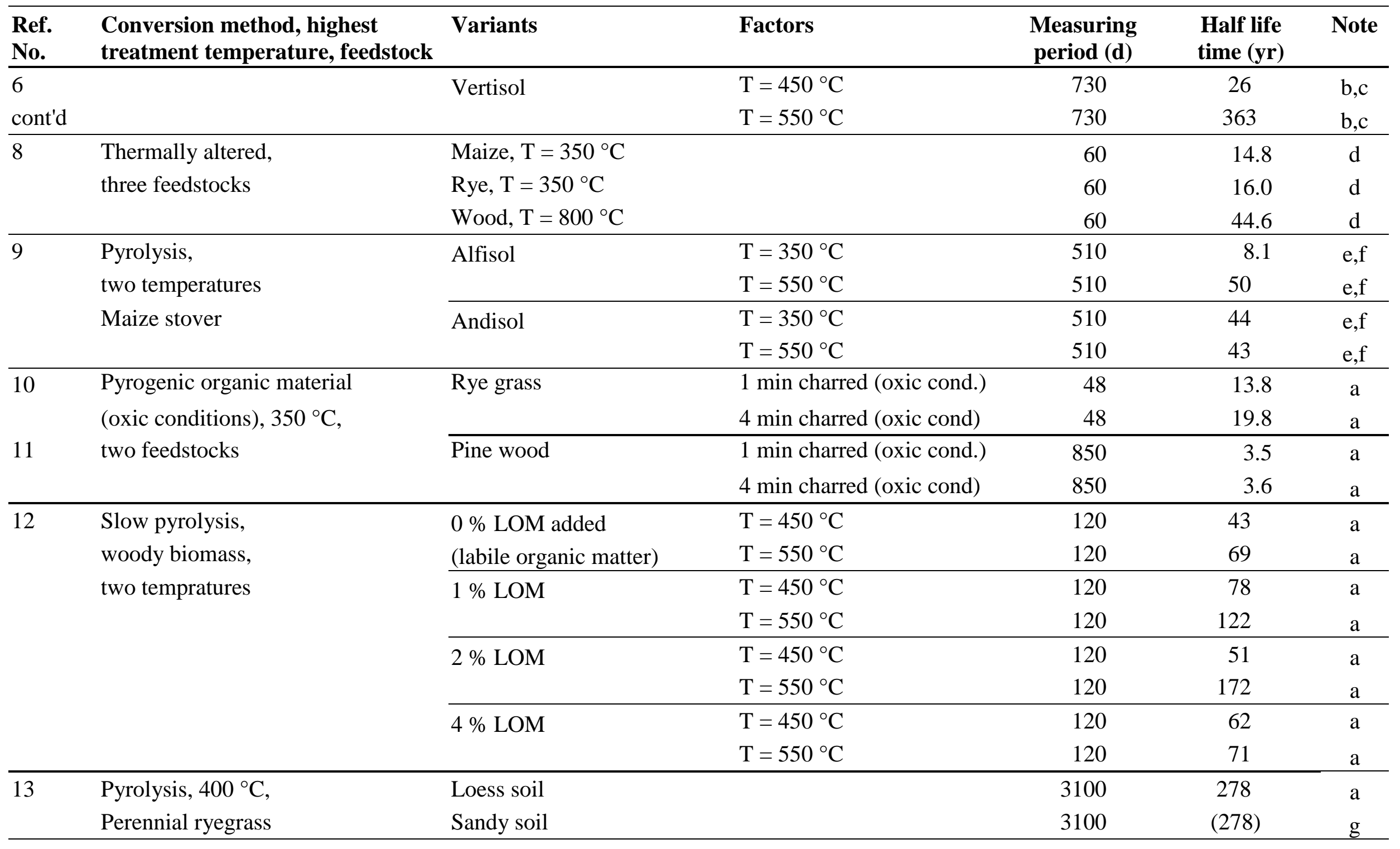




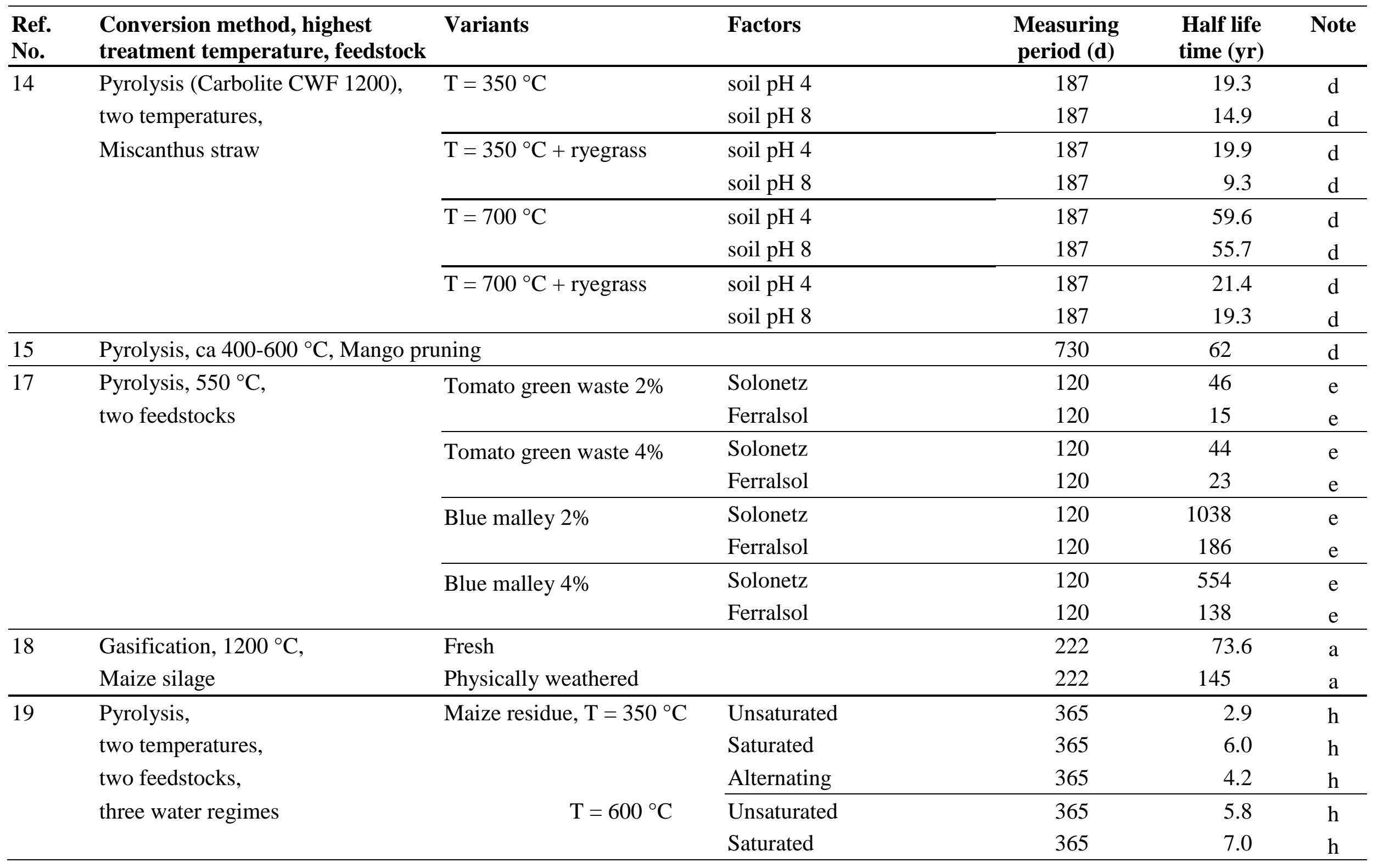




\begin{tabular}{|c|c|c|c|c|c|c|}
\hline $\begin{array}{l}\text { Ref. } \\
\text { No. }\end{array}$ & $\begin{array}{l}\text { Conversion method, highest } \\
\text { treatment temperature, feedstock }\end{array}$ & Variants & Factors & $\begin{array}{l}\text { Measuring } \\
\text { period }(d)\end{array}$ & $\begin{array}{l}\text { Half life } \\
\text { time (yr) }\end{array}$ & Note \\
\hline 19 & & & Alternating & 365 & 6.3 & $\mathrm{~h}$ \\
\hline \multirow[t]{3}{*}{$\operatorname{cont}^{6} d$} & & Oak wood, $\mathrm{T}=350^{\circ} \mathrm{C}$ & Unsaturated & 365 & 8.2 & $\mathrm{~h}$ \\
\hline & & & Alternating & 365 & 4.6 & $\mathrm{~h}$ \\
\hline & & $\mathrm{T}=600{ }^{\circ} \mathrm{C}$ & Unsaturated & 365 & 7.4 & $\mathrm{~h}$ \\
\hline \multirow[t]{2}{*}{20} & Kiln, ca. $500-600^{\circ} \mathrm{C}$, & $50 \mathrm{Mg} / \mathrm{ha}$ biochar & & 98 & 133 & $\mathrm{~h}$ \\
\hline & Wheat straw & $100 \mathrm{Mg} / \mathrm{ha}$ biochar & & 98 & 116 & $\mathrm{~h}$ \\
\hline \multirow[t]{3}{*}{21} & Charcoal, $550^{\circ} \mathrm{C}$, & Ferralsol & & 365 & 36.5 & $\mathrm{a}$ \\
\hline & Wood & Luvisol topsoil & & 365 & 9.5 & $\mathrm{a}$ \\
\hline & & Luvisol subsoil & & 365 & 11.4 & $\mathrm{a}$ \\
\hline \multirow[t]{9}{*}{22} & Pyrolysis, & Blue gum wood & $\mathrm{T}=400^{\circ} \mathrm{C}$, activated & 1829 & 226 & $\mathrm{a}$ \\
\hline & two temperatures, & & $\mathrm{T}=550^{\circ} \mathrm{C}$, activated & 1170 & 881 & $\mathrm{a}$ \\
\hline & five feedstocks & & $\mathrm{T}=400^{\circ} \mathrm{C}$, non-activated & 1829 & 204 & $\mathrm{a}$ \\
\hline & & & $\mathrm{T}=550^{\circ} \mathrm{C}$, non-activated & 1829 & 1120 & $\mathrm{a}$ \\
\hline & & Blue gum leaves & $\mathrm{T}=400^{\circ} \mathrm{C}$, activated & 1829 & 187 & $\mathrm{a}$ \\
\hline & & & $\mathrm{T}=550{ }^{\circ} \mathrm{C}$, activated & 1829 & 396 & $\mathrm{a}$ \\
\hline & & Paper sludge & $\mathrm{T}=550^{\circ} \mathrm{C}$, activated & 1829 & 274 & $\mathrm{a}$ \\
\hline & & Poultry manure & $\mathrm{T}=400^{\circ} \mathrm{C}$, non-activated & 1829 & 89 & $\mathrm{a}$ \\
\hline & & & $\mathrm{T}=550^{\circ} \mathrm{C}$, activated & 1829 & 217 & $\mathrm{a}$ \\
\hline
\end{tabular}




\begin{tabular}{|c|c|c|c|c|c|c|}
\hline $\begin{array}{l}\text { Ref. } \\
\text { No. }\end{array}$ & $\begin{array}{l}\text { Conversion method, highest } \\
\text { treatment temperature, feedstock }\end{array}$ & Variants & Factors & $\begin{array}{l}\text { Measuring } \\
\text { period }(d)\end{array}$ & $\begin{array}{l}\text { Half life } \\
\text { time (yr) }\end{array}$ & Note \\
\hline 22 & & Cow manure & $\mathrm{T}=400{ }^{\circ} \mathrm{C}$, non-activated & 1829 & 62 & $\mathrm{a}$ \\
\hline cont $^{6} \mathrm{~d}$ & & & $\mathrm{~T}=550^{\circ} \mathrm{C}$, activated & 1829 & 881 & $\mathrm{a}$ \\
\hline & two feedstocks & biochar & Forest soil & 175 & 6.0 & $\mathrm{a}$ \\
\hline & & Glucose-derived & Arable soil & 175 & 4.0 & $\mathrm{a}$ \\
\hline & Maize pellets & & UK & 164 & 4.8 & $\mathrm{a}$ \\
\hline \multirow[t]{3}{*}{26} & Pyrolysis, $450^{\circ} \mathrm{C}$, & $0.83 \%$ biochar added & $75 \%$ field capacity & 1095 & 14.1 & $\mathrm{~d}$ \\
\hline & Maize straw & & $100 \%$ field capacity & 1095 & 10.5 & $\mathrm{~d}$ \\
\hline & & $3.0 \%$ biochar added & $75 \%$ field capacity & 1095 & 15.8 & $\mathrm{~d}$ \\
\hline \multirow{9}{*}{27} & six feedstocks & & $\mathrm{T}=650^{\circ} \mathrm{C}$ & 365 & 15558 & $\mathrm{a}, \mathrm{i}$ \\
\hline & & Cedar & $\mathrm{T}=250^{\circ} \mathrm{C}$ & 365 & 733 & $\mathrm{a}, \mathrm{i}$ \\
\hline & & & $\mathrm{T}=400^{\circ} \mathrm{C}$ & 365 & 23815 & $\mathrm{a}, \mathrm{i}$ \\
\hline & & & $\mathrm{T}=650^{\circ} \mathrm{C}$ & 365 & $2 * 10^{7}$ & $\mathrm{a}, \mathrm{i}$ \\
\hline & & Grass & $\mathrm{T}=250^{\circ} \mathrm{C}$ & 365 & 263 & $\mathrm{a}, \mathrm{i}$ \\
\hline & & & $\mathrm{T}=400^{\circ} \mathrm{C}$ & 365 & 373 & $\mathrm{a}, \mathrm{i}$ \\
\hline & & & $\mathrm{T}=650^{\circ} \mathrm{C}$ & 365 & 152 & $\mathrm{a}, \mathrm{i}$ \\
\hline & & Oak & $\mathrm{T}=250^{\circ} \mathrm{C}$ & 365 & 843 & $\mathrm{a}, \mathrm{i}$ \\
\hline & & & $\mathrm{T}=400^{\circ} \mathrm{C}$ & 365 & 1021 & $\mathrm{a}, \mathrm{i}$ \\
\hline
\end{tabular}




\begin{tabular}{|c|c|c|c|c|c|c|}
\hline $\begin{array}{l}\text { Ref. } \\
\text { No. }\end{array}$ & $\begin{array}{l}\text { Conversion method, highest } \\
\text { treatment temperature, feedstock }\end{array}$ & Variants & Factors & $\begin{array}{l}\text { Measuring } \\
\text { period }(d)\end{array}$ & $\begin{array}{l}\text { Half life } \\
\text { time (yr) }\end{array}$ & Note \\
\hline 27 & & & $\mathrm{~T}=650^{\circ} \mathrm{C}$ & 365 & 96169 & $\mathrm{a}, \mathrm{i}$ \\
\hline \multirow[t]{3}{*}{$\operatorname{cont}^{\prime} \mathrm{d}$} & & Pine & $\mathrm{T}=400^{\circ} \mathrm{C}$ & 365 & 6786 & $\mathrm{a}, \mathrm{i}$ \\
\hline & & Sugar cane & $\mathrm{T}=250^{\circ} \mathrm{C}$ & 365 & 691 & $\mathrm{a}, \mathrm{i}$ \\
\hline & & & $\mathrm{T}=400^{\circ} \mathrm{C}$ & 365 & 9306 & $\mathrm{a}, \mathrm{i}$ \\
\hline \multirow[t]{2}{*}{1} & $\mathrm{HTC}, 180{ }^{\circ} \mathrm{C}$ & & Sandy soil & 200 & 1.0 & $\mathrm{a}$ \\
\hline & Miscanthus & & Loamy sand & 200 & 1.2 & $\mathrm{a}$ \\
\hline \multirow[t]{8}{*}{2} & HTC, Artec GmbH, & $\mathrm{T}=180^{\circ} \mathrm{C}$ & Sandy soil & 196 & 1.3 & $\mathrm{a}$ \\
\hline & two temperatures, & & Loamy sand & 196 & 1.0 & $\mathrm{a}$ \\
\hline & & Washed biochar & Sandy soil & 196 & 1.6 & $\mathrm{a}$ \\
\hline & & & Loamy sand & 196 & 1.5 & $\mathrm{a}$ \\
\hline & HTC, Fa. Schlitt $200^{\circ} \mathrm{C}$, & & Sandy soil & 196 & 1.2 & $\mathrm{a}$ \\
\hline & Maize silage & & Loamy sand & 196 & 1.3 & $\mathrm{a}$ \\
\hline & HTC, MPI Potsdam, $200^{\circ} \mathrm{C}$, & & Sandy soil & 196 & 6.5 & $\mathrm{a}$ \\
\hline & Maize silage & & Loamy sand & 196 & 5.8 & $\mathrm{a}$ \\
\hline \multirow[t]{3}{*}{5} & $\mathrm{HTC}, 230^{\circ} \mathrm{C}$, & Wheat straw & Topsoil & 500 & 13.3 & $\mathrm{e}$ \\
\hline & Two feedstocks & & Subsoil & 500 & 18.0 & $\mathrm{e}$ \\
\hline & & Wheat straw + digestate & Topsoil & 500 & 13.8 & $\mathrm{e}$ \\
\hline
\end{tabular}




\begin{tabular}{|c|c|c|c|c|c|c|}
\hline $\begin{array}{l}\text { Ref. } \\
\text { No. }\end{array}$ & $\begin{array}{l}\text { Conversion method, highest } \\
\text { treatment temperature, feedstock }\end{array}$ & Variants & Factors & $\begin{array}{c}\text { Measuring } \\
\text { period }(d)\end{array}$ & $\begin{array}{l}\text { Half life } \\
\text { time (yr) }\end{array}$ & Note \\
\hline 5 cont $^{6} \mathrm{~d}$ & & & Subsoil &. .500 & 20.3 & $\mathrm{e}$ \\
\hline \multirow[t]{5}{*}{7} & HTC, & Draff & $\mathrm{T}=180^{\circ} \mathrm{C}, 4 \mathrm{~h}$ & 248 & 0.5 & $\mathrm{a}$ \\
\hline & two durations, & & $\mathrm{T}=200^{\circ} \mathrm{C}, 4 \mathrm{~h}$ & 248 & 0.9 & $\mathrm{a}$ \\
\hline & two feedstocks & & $\mathrm{T}=250^{\circ} \mathrm{C}, 4 \mathrm{~h}$ & 248 & 1.7 & $\mathrm{a}$ \\
\hline & & & $\mathrm{T}=200{ }^{\circ} \mathrm{C}, 4 \mathrm{~h}$ & 248 & 0.5 & $\mathrm{a}$ \\
\hline & & & $\mathrm{T}=250^{\circ} \mathrm{C}, 4 \mathrm{~h}$ & 248 & 1.8 & $\mathrm{a}$ \\
\hline \multirow[t]{2}{*}{16} & $\mathrm{HTC}, 230^{\circ} \mathrm{C}$ & Coarse soil & & 365 & 8.8 & $\mathrm{a}$ \\
\hline & Maize silage & Fine soil & & 365 & $(8.8)$ & $\mathrm{k}$ \\
\hline 18 & $\mathrm{HTC}, 250^{\circ} \mathrm{C}$ & fresh & & 196 & 8.4 & $\mathrm{a}$ \\
\hline 21 & & Luvisol subsoil & & 365 & 9.5 & $\mathrm{a}$ \\
\hline \multirow[t]{7}{*}{22} & HTC & Poplar & $\mathrm{T}=230^{\circ} \mathrm{C}$, washed & 120 & 9.2 & $\mathrm{e}, 1$ \\
\hline & two feedstocks & Straw digestate & $\mathrm{T}=210^{\circ} \mathrm{C}$, washed & 120 & 2.6 & $\mathrm{e}, 1$ \\
\hline & three temperatures (for straw & & $\mathrm{T}=230^{\circ} \mathrm{C}$, washed & 120 & 9.3 & $e, 1$ \\
\hline & digestate) & & $\mathrm{T}=250^{\circ} \mathrm{C}$, washed & 120 & 9.4 & $e, 1$ \\
\hline & & & $\mathrm{T}=210^{\circ} \mathrm{C}$, unwashed & 120 & 2.3 & $\mathrm{e}, 1$ \\
\hline & & & $\mathrm{T}=230^{\circ} \mathrm{C}$, unwashed & 120 & 6.2 & $\mathrm{e}, 1$ \\
\hline & & & $\mathrm{T}=250^{\circ} \mathrm{C}$, unwashed & 120 & 9.4 & $\mathrm{e}, 1$ \\
\hline
\end{tabular}




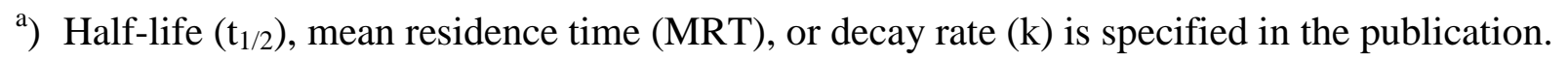

b) $t_{1 / 2}$ calculated by the authors with a two component (double exponential) decomposition model based on the published data on carbon loss over time.

c) Only variants with $20^{\circ} \mathrm{C}$ incubation temperature accounted for statistical analysis.

d) $t_{1 / 2}$ calculated by the authors with a one component (single exponential) decomposition model based on the published data on carbon loss over time.

e) $t_{1 / 2}$ calculated by the authors based on published parameters of the respective decomposition model (one, two, or three components).

f) $t_{1 / 2}$ calculated with parameters of a three component model (standardized for $19{ }^{\circ} \mathrm{C}$ ).

g) Exact result not given, but reported in the text as very similar to the loess variant.

h) $t_{1 / 2}$ calculated by the authors with a one component (single exponential) model based on published C loss at the end of measurement period.

i) Data of Zimmermann (2010) additionally reported but not accounted for statistical analysis of half-life quantiles due to the artificial.

$\left.{ }^{\mathrm{k}}\right)$ Half-life for variant fine soil not explicitly reported, but stated as nearly identical to variant coarse soil.

l) 5 variants with recirculated process liquor not respected. 


\section{References for Table S5}

1 Bai M, Wilske B, Buegger F et al. Degradation kinetics of biochar from pyrolysis and hydrothermal carbonization in temperate soils. Plant Soil 372, 375-387 (2013).

2 Bai M, Wilske B, Bach M, Breuer L. Entwicklung eines Routineverfahrens zur Bestimmung der Abbaurate von Biokohlen im Boden Development of a routine procedure for determination of biochar decomposition rate in soils. Final Report, Deutsche Bundesstiftung Umwelt, Osnabrück, Germany, Project No. 29031(2014).

3 Bruun EW, Hauggaard-Nielsen H, Ibrahim I, Egsgaard H et al. Influence of fast pyrolysis temperature on biochar labile fraction and short-term carbon loss in a loamy soil. Biomass Bioenergy 35(3), 1182-1189 (2011).

4 Cayuela ML, Oenema O, Kuikman PJ, Bakker RR, van Groeningen, JW. Bioenergy byproducts as soil amendments? Implications for carbon sequestration and greenhouse gas emissions. GCB Bioenergy 2, 201-213 (2010).

5 Dicke C, Lanze G, Mumme J, Ellerbrock R, Kern J. Effect of Hydrothermally carbonized char application on trace gas emission from two sandy soils. J. Environmental Quality 43, 1790-1798 (2014).

6 Fang Y, Singh B, Singh BP, Krull E. Biochar carbon stability in four contrasting soils. European Journal Soil Science 65, 60-71 (2014).

7 Gajic A, Ramke HR, Hendricks A, Koch HJ. Microcosm study on the decomposability of hydrochars in a Cambisol. Biomass Bioenergy 47, 250-259 (2012).

8 Hamer U, Marschner B, Brodowski S, Amelung W. Interactive priming of black carbon and glucose mineralization. Organic Geochemistry 35, 823-830 (2004).

9 Herath HMS, Herath K, Camps-Arbestain M, Hedley MJ, Kirschbaum MUF, Wang T, van Hale R. Experimental evidence for sequestering $\mathrm{C}$ with biochar by avoidance of $\mathrm{CO}_{2}$ emissions from original feedstock and protection of native soil organic matter, $G C B$ Bioenergy, DOI: 10.1111/gcbb.12183 (2014).

10 Hilscher A, Heister K, Siewert C, Knicker H. Mineralisation and structural changes during the initial phase of microbial degradation of pyrogenic plant residues in soil. Organic Geochemistry 40, 332-342 (2009).

11 Hilscher A, Knicker. Biochar mineralization and priming effect on SOM decomposition in two European short rotation coppices. Organic Geochemistry 42, $42-54$ (2011).

12 Keith A, Singh B, Singh BP. Interactive Priming of Biochar and Labile Organic Matter Mineralization in a Smectite-Rich Soil. Environmental Science Technology 45(22), 96119618 (2011)

13 Kuzyakov Y, Bogomolova I, Glaser B. Biochar stability in soil: Decomposition during eight years and transformation as assessed by compound-specific 14C analysis. Soil Biology Biochemistry 70, 229-236 (2014).

14 Luo Y, Durenkamp M, De Nobili M, Lin Q. Short term soil priming effects and the mineralisation of biochar following its incorporation to soils of different pH. Soil Biology Biochemistry 43, 2304-2314 (2011).

15 Major J, Lehmann J, Rondon M, Goodale C. Fate of soil-applied black carbon: downward migration, leaching and soil respiration. Global Change Biology 16, 1366-1379 (2010). 
16 Malghani S, Jüschke E, Baumert J, Thuille A et al. Carbon sequestration potential of hydrothermal carbonization char (hydrochar) in two contrasting soils; results of a 1-year field study. Biology Fertility Soils 51, 123-134 (2015).

17 Murray J, Keith A, Singh B. The stability of low- and high-ash biochars in acidic soils of contrasting mineralogy. Soil Biology Biochemistry 89, 217-225 (2015).

18 Naisse C, Girardin C, R Lefevre, A Pozzi A, Maas R, Stark A. Effect of physical weathering on the carbon sequestration potential of biochars and hydrochars in soil. $G C B$ Bioenergy, DOI: 10.1111/gcbb.12158 (2014).

19 Nguyen BT, Lehmann J. Black carbon decomposition under varying water regimes. Organic Geochemistry 40, 846-853 (2009).

20 O’Toole A, Knoth de Zarruk K, Steffens M, Rass DP. Characterization, Stability, and Plant Effects of Kiln-Produced Wheat Straw Biochar. Journal Environmental Quality 42, 429-436 (2013).

21 Qayyum MF, Steffens D, Reisenauer HP, Schubert S. Kinetics of Carbon Mineralization of Biochars Compared with Wheat Straw in Three Soils. Journal Environmental Quality 41, 1210-1220 (2012).

22 Schulze M, Mumme J, Funke A, Kern J. Effetc of selected process conditions on the stability of hydrochar in low-carbon sandy soils. Geoderma 267, 137-145 (2016)

23 Singh BP, Cowie Al, Smernik RJ. Biochar carbon stability in a clayey soil as a function of feedstock and pyrolysis temperature. Environmental Science Technology 46, 1177011778 (2012).

24 Steinbeiss S, Gleixner G, Antonietti M. Effect of biochar amendment on soil carbon balance and soil microbial activity. Soil Biology Biochemistry 41, 1301-1310 (2009).

25 Ventura M, Alberti G, Viger M. Jenkins JR. Biochar mineralization and priming effect on SOM decomposition in two European short rotation coppices. GCB Bioenergy 7, 11501160 (2015).

26 Zhang MK, Gu GP, Wang Y. Degradation characteristic of different biochar materials in soil environments. Journal of Zhejiang University, Hangzhou, China (Agriculture and Life Science) 38, 329-335 (2012).

27 Zimmerman AR. Abiotic and microbial oxidation of laboratory-produced black carbon (biochar). Environmental Science Technology 44, 1295-1301 (2010). 
Table S6. Median and quantiles of biochar half-lifes from soil incubation experiments (data of Table S5).

\begin{tabular}{|c|c|c|c|c|c|c|}
\hline \multirow[t]{2}{*}{ Biochar type } & \multicolumn{5}{|c|}{ Quantile } & \multirow[t]{2}{*}{$\mathbf{n}$} \\
\hline & $5 \%$ & $25 \%$ & $\mathbf{5 0 \%}$ & $75 \%$ & $95 \%$ & \\
\hline & \multicolumn{5}{|c|}{ - } & \\
\hline $\begin{array}{l}\text { Pyrolysis - without data } \\
\text { of Zimmermann (2010) }\end{array}$ & 3.0 & 8.0 & 20.6 & 89.8 & 547 & 104 \\
\hline $\begin{array}{l}\text { Pyrolysis - including data } \\
\text { of Zimmermann (2010) }\end{array}$ & 3.5 & 10.1 & 44.1 & 226 & 6786 & 121 \\
\hline Hydrothermal biochar (HTC) & 0.5 & 1.3 & 2.9 & 9.3 & 18.1 & 40 \\
\hline
\end{tabular}

a) Experimental setup of Zimmermann (2010, see ref. 27 to Table S5) did not use natural soil but pure quartz sand with microbial inoculum; estimation of $t_{1 / 2}$ by decay function with decay rate $k$ declining over time. 